\title{
Users First: User-Centric Cluster Formation for Interference-Mitigation in Visible-Light Networks
}

\author{
Xuan Li, Fan Jin, Rong Zhang, Member, IEEE, Jiaheng Wang, Senior Member, IEEE, Zhengyuan Xu, \\ and Lajos Hanzo, Fellow, IEEE
}

Abstract-Visible light communication (VLC) combined with advanced illumination may be expected to become an integral part of next-generation heterogeneous networks. In order to mitigate the performance degradation imposed by the intercellinterference (ICI), a user-centric (UC) cluster formation technique employing vectored transmission (VT) is proposed for the VLC down-link system, where multiple users may be simultaneously supported by multiple access points (APs). In contrast to the traditional network-centric (NC) design, the UC-VT cluster formation is dynamically constructed and adjusted, rather than remaining static. Furthermore, we consider the critical issue of multiuser scheduling (MUS) relying on maximizing the "sum utility" of this system, which leads to a joint cluster formation and MUS problem. In order to find a practical solution, the original problem is reformulated as a maximum weighted matching (MWM) problem relying on a user-AP distance-based weight and then a low-complexity greedy algorithm is proposed, which offers a suboptimal yet compelling solution operating close to the optimal value found by the potentially excessive-complexity exhaustive search. Our simulation results demonstrate that the proposed greedy MUS algorithm combined with the UC-VT cluster formation is capable of providing an average user throughput of about $\mathbf{9 0 \%}$ of the optimal throughput, which is about three times the throughput provided by the traditional cellular design in some of the scenarios considered.

Index Terms-Visible light communication, user-centric cluster formation, multi-user scheduling, maximum weighted matching.

$\mathbf{O}$ WING to its huge unlicensed bandwidth, high data rate potential, energy-efficient illumination etc., the research of Visible Light Communication (VLC) intensified during the past decade or so [1]. As a complementary extension of classic radio frequency communications, extensive investigations have

Manuscript received April 9, 2015; revised July 20, 2015; accepted August 3,2015 . This work was supported by the RC-UK under the auspices of the UKIndia ATC in Wireless Communications, of the Chinese Scholarship Council as well as of the European Research Council's Advanced Fellow Grant. The work of J. Wang was supported in part by the 973 Program of China under Grant 2013CB336600, in part by the NSFC under Grant 61201174, in part by the NSF of Jiangsu under Grant BK2012325, and in part by the Fundamental Research Funds for the Central Universities. The associate editor coordinating the review of this paper and approving it for publication was Dr. Peter Rost.

X. Li, F. Jin, R. Zhang, and L. Hanzo are with the University of Southampton, Southampton, U.K. (e-mail: x126g12@ecs.soton.ac.uk; fj1g10@ ecs.soton.ac.uk; rz@ecs.soton.ac.uk; lh@ecs.soton.ac.uk).

J. Wang is with the Southeast University, Nanjing, China (e-mail: jhwang@seu.edu.cn).

Z. Xu is with the University of Science and Technology of China, Hefei, China (e-mail: xuzy@ustc.edu.cn).

Color versions of one or more of the figures in this paper are available online at http://ieeexplore.ieee.org.

Digital Object Identifier 10.1109/TWC.2015.2466539 been dedicated to the point-to-point transmission and reception techniques in VLC networks [2]-[10], as also indicated by the IEEE 802.15 .7 standard ratified for short-range visible light wireless communication [11]. Apart from their multifold advantages, naturally, VLC systems also exhibit several potential drawbacks, such as reduced performance in non-lineof-sight scenarios, lack of native up-link support, a confined coverage compared to cellular radio frequency networks etc.

Amongst all the design challenges, the performance degradation imposed by Inter-Cell Interference (ICI) at the cell edge may lead to dramatic reduction of the Quality of Service (QoS) in a VLC down-link system. As a result, careful VLC cell formation becomes crucial, since it is the salient design stage of the entire system design cycle. Traditional designs conceived for VLC cells operating both with and without Frequency Reuse (FR) or fractional frequency reuse planning, have been studied in [12]-[14], where each optical Access Point (AP) illuminates a small confined cell. As a further advance, a multi-AP joint transmission scheme relying on Combined Transmission (CT) and Vectored Transmission (VT) ${ }^{1}$ were also investigated in [12]. In contrast to the above-mentioned Network-Centric (NC) design philosophy, a novel User-Centric (UC) cell formation regime was proposed in [15]-[17], where amorphous user-specific multi-AP cells are constructed for jointly transmitting data to a single User Equipment (UE) ${ }^{2}$ by employing $\mathrm{CT}$, which we referred to as UC-CT. By definition, UC design is different from the NC design, where the network configuration is fixed, regardless of the tele-traffic. In order to further improve the achievable bandwidth efficiency of the previously proposed UC-CT and to allow each multi-AP cell simultaneously serve multiple UEs as discussed in [12], we propose the UC-VT-based cluster formation principle in this paper. UC-VT cluster formation may be defined as forming the UC-VT clusters, where each UC-VT cluster is served by a set of VLC APs, which simultaneously serve multiple UEs by employing VT. More explicitly, a UC-VT cluster includes a set of APs and UEs as well as the transmission links between them. Note that the previously proposed UC-CT-based cell formation of [15]-[17]

\footnotetext{
${ }^{1}$ In [12], relying on CT, each individual VLC AP of a multi-AP cell conveyed the same information on the same visible carrier frequency in their overlapping areas and served a single user at a time. In order to eliminate the bandwidth efficiency reduction imposed by CT, Zero-Forcing (ZF)-based VT techniques were employed for serving multiple users at the same time in the overlapping area, which will be exemplified in Section II-C

${ }^{2} \mathrm{~A}$ single UE represents a communication device equipped with a VLC receiver in our down-link VLC system, which could be a smart phone, a personal computer, a tablet, a printer, etc.
} 
may be regarded as a special case of our UC-VT-based cluster formation, when only a single UE resides within the coverage of the UC-VT cluster.

When multiple UEs are present in a VLC network, efficient resource allocation and Multi-User Scheduling (MUS) constitutes one of the salient problems, which in fact affects all multi-user networks. However, the problem of VLC-based networks has remained to a large extent hitherto unexplored in the open literature, although recently some valuable studies were disseminated in the context of NC single-AP VLC cells [18]-[21]. In particular, the authors of [18] proposed a heuristic scheme for allocating interference-constrained sub-carriers in a multiple access VLC system relying on Discrete MultiTone (DMT) modulation, in order to improve the aggregate throughput. The authors of [19] carefully designed a logical framework aiming to localize, access, schedule and transmit in VLC systems, which was capable of achieving a substantial throughput at a modest complexity. However, similar to most of the literature studying resource allocation in VLC-based systems, both [18] and [19] endeavour to improve the attainable throughput without giving any cognizance to the fairness experienced by the UEs. By taking fairness into account, the authors of [20] proposed an Incremental Scheduling Scheme (ISS), where the global scheduling phase is responsible for assigning the resources to the UEs, while the local scheduling phase regularly adjusts the resource allocation by backtracking the UEs' movements. Furthermore, the authors of [21] proposed a Proportional Fairness (PF) based scheduling algorithm for a centrally controlled VLC system, which outperformed the maximum-rate scheduling policy in terms of balancing the achievable throughput against the fairness experienced by the UEs. Broadly speaking, most studies of the MUS problem encountered in VLC systems are based on single-AP VLC cells. By contrast, we are going to tackle the problems of MUS and UC-based cluster formation relying on VT.

Against the above-mentioned background, in this paper,

i) we investigate the MUS problem relying on the UEs' $\mathrm{PF}$ as a measure by assigning each UE a specific scheduling priority, which is inversely proportional to its anticipated resource consumption [22] and then maximizing a carefully selected network utility function [23], when jointly considering amorphous UC-VT cluster formations for the VLC down-link.

ii) More explicitly, the optimal solution of this joint UCbased cluster formation and MUS problem is first found by a high-complexity exhaustive search, which may have an overwhelming complexity even for a modest-scale system. In order to reduce the computational complexity, the original problem is formulated as a Maximum Weighted Matching (MWM) problem and multiple UEs are scheduled by solving the Kuhn-Munkres (KM) algorithm [24]-[28].

iii) To further improve the grade of practicability, a greedy algorithm is proposed, which operates at a considerably lower complexity, despite taking into account the dynamics of the UC-VT clusters.

iv) Moreover, the computational complexity of both the exhaustive search and of the proposed schemes is analysed and various cluster formations are evaluated for 134 diverse VLC characteristics, such as the Field-Of-View 135 (FOV), the Line-Of-Sight (LOS) blocking probabilities, 136 the optical AP arrangement, etc. 137

VLC can be considered as a new member in the small-cell 138 family of the Heterogeneous Network (HetNets) landscape for 139 complementing the overloaded radio frequency band [17]. The 140 UC cluster formation principle designed for VLC environments 141 constitutes a novel and competitive design paradigm for the 142 super dense multi-tier cell combinations of HetNets, where 143 the sophisticated UEs can actively participate in cell planning, 144 resource management, mobility control, service provision, sig- 145 nal processing, etc. Considering the large-scale multi-input- 146 multi-output systems for example, the antenna selection scheme 147 or beamforming techniques may be designed in a similar UC 148 manner, according to the UEs' geo-location and service require- 149 ments. As a result, the UC concept may be expected to become 150 one of the disruptive techniques to be used in the forthcoming 151 $5 \mathrm{G}$ era [17].

The rest of this paper is organized as follows. Our system 153 model and the UC-VT clusters considered are presented in 154 Section II. Our MUS methodology is described and evaluated 155 in Section III and Section IV, respectively, while dynamically 156 constructing UC-VT clusters. Finally, Section V offers our 157 conclusions.

\section{SYSTEM MODEL}

The VLC down-link is considered, which is constituted by a 160 set of VLC APs and each of them relies on an LED array con- 161 structed from several LEDs. The essence of our UC-VT cluster 162 formation is to assign the UEs and optical APs to each other for 163 the sake of maximizing the total utility after employing VT in 164 each of the UC-VT cluster. This procedure is entirely based on 165 the UEs' specific conditions and thus leads to UC clusters. In 166 this section, we first discuss the optical link characteristics and 167 cluster formation, before investigating how to select the UE set 168 supported by a specific AP set in a multi-user system.

\section{A. Link Characteristics}

Since each UE has a limited FOV, they can only receive infor- 171 mation from the optical APs, when one or more APs reside 172 within the UE's FOV. According to [29], if the angle of inci- 173 dence $\psi$ from an AP to a UE is less than the UE's FOV $\psi_{\mathrm{F}}$, the 174 optical channel's Direct Current (DC) attenuation of the LOS 175 path is given by

$$
h_{d}=\frac{(m+1) D_{\mathrm{PA}}}{2 \pi l^{2}} \cos ^{m}(\phi) T_{S}(\psi) g(\psi) \cos (\psi),
$$

where the Lambert index $m$ depends on the semi-angle $\phi_{1 / 2} 177$ at half-illuminance of the source, which is given by $m=178$ $-1 / \log _{2}\left(\cos \phi_{1 / 2}\right)$. Furthermore, $D_{\mathrm{PA}}$ is the physical area of 179 the detector's Photo-Diode (PD), $l$ is the distance between 180 the VLC transmitter and the receiver, while $\phi$ is the angle 181 of irradiance. Still referring to $(1), T_{s}(\psi)$ and $g(\psi)$ denote 182 the gain of the optical filter and of the optical concentrator 183 
TABLE I

VLC PARAMETERS

\begin{tabular}{l|r}
\hline \hline Semi-angle at half power $\left(\phi_{1 / 2}\right)$ & $70^{\circ}$ \\
Modulation bandwidth $(B)[7]$ & $20[\mathrm{MHz}]$ \\
Physical area of a PD $\left(D_{\mathrm{PA}}\right)$ & $1.0\left[\mathrm{~cm}^{2}\right]$ \\
Gain of an optical filter $\left(T_{s}(\psi)\right)$ & 1.0 \\
Refractive index of a lens at a PD $(n)$ & 1.5 \\
O/E conversion efficiency $(\gamma)$ & $0.53[\mathrm{~A} / \mathrm{W}]$ \\
\hline \hline
\end{tabular}

employed, respectively, while $g(\psi)$ can be written as $g(\psi)=$ $n^{2} / \sin ^{2} \psi_{\mathrm{F}}$ [29], where $n$ is the refractive index of a lens at a PD. Furthermore, according to [10], when the incidence angle $\psi$ is no larger than the FOV, the channel's DC attenuation on the first reflection is given by

$$
\begin{aligned}
d h_{r}= & \frac{(m+1) D_{\mathrm{PA}}}{2 \pi^{2} l_{1}^{2} l_{2}^{2}} \rho d D_{\text {wall }} \cos ^{m}(\phi) \cos \left(\beta_{1}\right) \\
& \cos \left(\beta_{2}\right) T_{s}(\psi) g(\psi) \cos (\psi),
\end{aligned}
$$

where $l_{1}$ denotes the distance between an AP and a reflective point, while $l_{2}$ is the distance between this point and a UE. The reflectance factor and the reflective area are denoted by $\rho$ and $d D_{\text {wall }}$, respectively. Additionally, $\beta_{1}$ and $\beta_{2}$ represent the irradiance angles to the reflective point and to the UE, respectively. Our VLC parameter values are summarized in TABLE I.

\section{B. Cluster Formation}

Following the traditional cellular design principle, each optical AP illuminates an individual cell and adopts Unity Frequency Reuse (UFR) across all cells, where the ICI is imposed by the LOS ray of neighbouring cells and consequently the UE may experience dramatic performance degradation at the cell edge. In order to reduce the ICI, appropriate FR patterns may be employed as an appealingly simple solution, while the system has to obey the classic trade-off between reduced bandwidth efficiency and improved cell-edge Signalto-Interference-plus-Noise-Ratio (SINR), when using a FR factor higher than one, as investigated in our previous work [12]. Apart from the single-AP cells, we studied multi-AP merged cells, where several neighbouring VLC APs cooperate by employing either CT or VT techniques. The above-mentioned cell designs, including regular UFR/FR and merged multi-AP cells with CT/VT, rely on a fixed cell-shape, regardless of the traffic requirements, which are referred to as $\mathrm{NC}$ formations. In contrast to the fixed-shape NC cell formation designs, the UC design philosophy was proposed in [15]-[17], which was capable of supporting irregular-shape elastic cell formations that were capable of accommodating dynamic traffic requirements. By employing CT, each multi-AP UC-CT cell of [15] is only capable of supporting a single UE in a specific time slot. In order to serve multiple UEs at the same time, we propose the VT aided UC cluster formation, which is referred to as a UCVT cluster in this paper. Let us now discuss the model of our system in more detail.

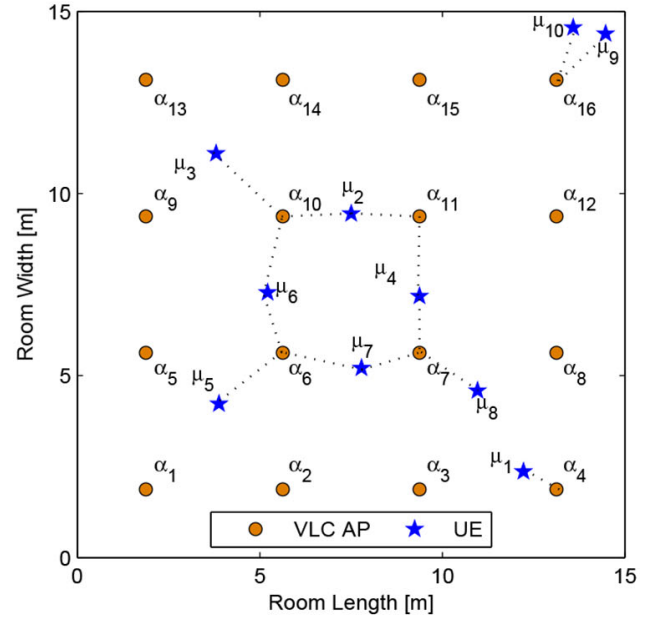

(a)

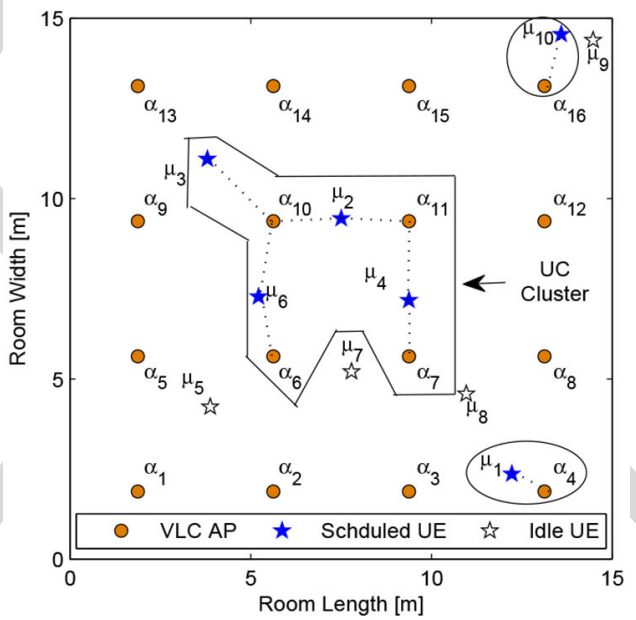

(b)

Fig. 1. (a) Layout of the VLC APs and UEs projected on the horizontal plane, where $\alpha_{i}$ and $\mu_{j}$ represent the VLC APs and UEs, respectively. All LOS links are denoted by dotted lines and for simplicity, the reflections are not shown in this figure. There are $(4 \times 4)=16$ APs and 10 UEs. (b) The cluster formation result provided by Fig. 5d for the VLC system of (a).

Fig. 1a shows the example of a particular VLC down-link 223 network having $N_{A}=16$ optical APs and $N_{U}=10 \mathrm{UEs}$, where 224 all LOS links are denoted by dotted lines and for simplicity, 225 the reflections are not shown. Let us first construct the link's 226 bipartite graph $\mathcal{G}(\mathcal{V}, \mathcal{E})$, as shown in Fig. 2a, for the network of 227 Fig. 1a. The vertex set $\mathcal{V}$ denoting the communication nodes is 228 divided into two subsets, i.e. the optical AP set $V_{A}$ as well as 229 the VLC UE set $\mathcal{V}_{U}$, where we have

$$
\begin{aligned}
\mathcal{V} & =\mathcal{V}_{A} \cup \mathcal{V}_{U} \\
& =\left\{\alpha_{i} \mid i=1,2, \ldots, N_{A}\right\} \cup\left\{\mu_{j} \mid j=1,2, \ldots, N_{U}\right\},
\end{aligned}
$$

with $\alpha_{i}$ and $\mu_{j}$ denoting the index of VLC APs and UEs, 231 respectively. Hence, the number of vertices in $\mathcal{G}$ is given by 232 $\left(N_{A}+N_{U}\right)$. Furthermore, when a UE can receive data from an 233 AP, either via the direct LOS path or via the reflected path, a 234 link may be established between them, which is said to be an 235 edge, and these two vertices are said to be adjacent. The edge 236 set $\mathcal{E}$ represents all possible links between APs and UEs with 237 


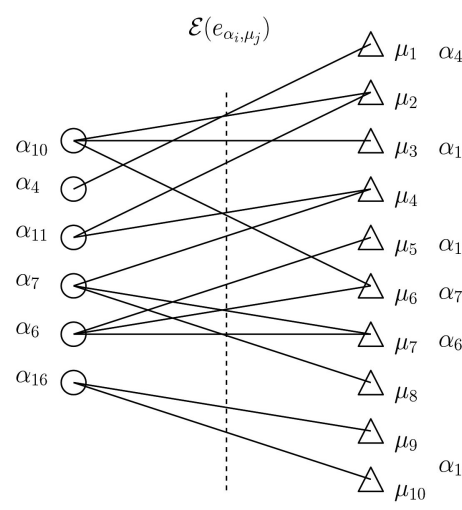

$\operatorname{VLC} \operatorname{AP}\left(\mathcal{V}_{A}\right)$

(a)
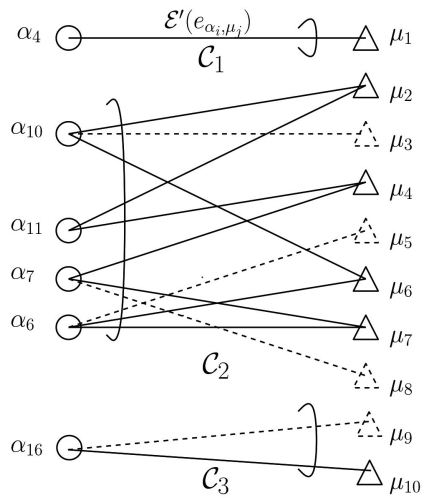

$\mu_{9}$

$\operatorname{VLC}\left(\mathcal{V}_{A}^{\prime}\right)$

(c)

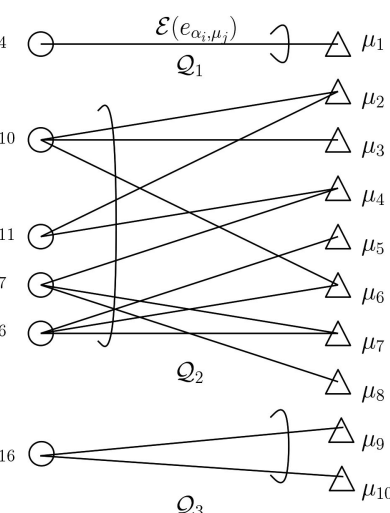

$\mathrm{UC}\left(\mathcal{V}_{U}\right)$

(b)
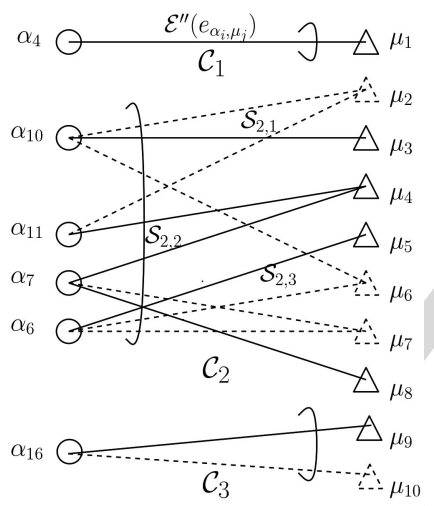

$\mathrm{UE}\left(\mathcal{V}_{U}^{\prime \prime}\right)$

(d)

Fig. 2. (a) A graph model $\mathcal{G}(\mathcal{V}, \mathcal{E})$ of the VLC down-link seen in Fig. 1a. (b) The three independent components of $\mathcal{G}$, i.e. $\mathcal{Q}_{1}, Q_{2}$ and $\mathcal{Q}_{3}$. (c) and (d) Possible UC-VT cluster formations of the network. In (d), $\mathcal{S}_{2,1}, \mathcal{S}_{2,2}$ and $\mathcal{S}_{2,3}$ are disjoint, but they are regarded as a merged large cluster $\mathcal{C}_{2}$.

one of the endpoints in $V_{A}$ and the other one in $V_{U}$, which may be written as

$$
\mathcal{E}=\left\{e_{\alpha_{i}, \mu_{j}} \mid \alpha_{i} \in \mathcal{V}_{A}, \mu_{j} \in \mathcal{V}_{U}\right\}
$$

where $e_{\alpha_{i}, \mu_{j}}$ denotes the link between AP $\alpha_{i}$ and UE $\mu_{j}$. Since the placement of the VLC APs is fixed, the edge set is determined by the UEs' specific conditions, such as their FOV, position, etc. Therefore, the network graph is said to be UC.

Still referring to Fig. 2a, the graph $\mathcal{G}$ is not fully connected, since not all pairs of vertices are joined by a path. Further scrutiny reveals that $\mathcal{G}$ has three independent components, which are said to be partially connected components, as explicitly shown in Fig. $2 \mathrm{~b}$, marked by $\mathcal{Q}_{1}, \mathcal{Q}_{2}$ and $\mathcal{Q}_{3}$. There are no adjacent AP-UE vertices amongst these distinctive components of $2_{1}, 2_{2}$ and $2_{3}$, which indicates that UEs cannot receive data from the optical APs belonging to the other components, only from their own. Thus, the ICI is totally eliminated. Explicitly, since none of the individual components is affected by the others, the proposed cluster formation algorithms may be executed within every single component, as it will be discussed in Section III. On the other hand, in order to simultaneously serve multiple UEs, Zero-Forcing (ZF)-based
VT techniques are introduced in our system. The underlying 258 principle of ZF-based VT is to totally eliminate the interfer- 259 ence at the multiple AP transmitters, so that all the UEs receive 260 mutually interference-free signals. In general, when employing 261 VT the maximum number of UEs supported in a single time 262 slot should be no more than the number of APs. Hence, the ZF- 263 based VT may not be employed directly by each component in 264 Fig. 2b. For example, the number of UEs is almost twice as high 265 as the number of APs in $Q_{2}$. Therefore, we eliminate the inter- 266 ference by ensuring that only some of the UEs will be scheduled 267 and we solve this problem by constructing a UC-VT cluster 268 with the aid of the serving APs. There are various options for 269 scheduling the UEs shown in Fig. 2c and 2d, where the UEs 270 denoted by the dashed triangle boundary are not scheduled and 271 the edges denoted by dashed lines are not established during 272 the current slot. Furthermore, the UC-VT clusters formed are 273 denoted by $\mathcal{C}_{n}$, i.e. by $\mathcal{C}_{1}, \bigodot_{2}$ and $\bigodot_{3}$ in Fig. 2 c and 2 d. Before 274 investigating how to schedule the UEs, let us first discuss the 275 VT within each UC-VT cluster formed.

\section{Vectored Transmission}

After scheduling the UEs, each UC-VT cluster is formed, as 278 shown for example in Fig. 2c, where the clusters are denoted by 279 $\mathrm{C}_{1}, \mathrm{C}_{2}$ and $\mathrm{C}_{3}$, respectively. Within $\mathrm{C}_{1}$ or $\mathrm{C}_{3}$, only a single $\mathrm{UE}$ is 280 supported by a single AP, which is a similar scenario to the reg- 281 ular NC design. However, in order to allow $\left\{\alpha_{10}, \alpha_{11}, \alpha_{7}, \alpha_{6}\right\} 282$ to simultaneously serve all the UEs $\left\{\mu_{2}, \mu_{4}, \mu_{6}, \mu_{7}\right\}$ within 283 $\mathcal{C}_{2}$, we employ Zero-Forcing (ZF)-based VT techniques. More 284 explicitly, we may write the channel's attenuation $\boldsymbol{H}_{\mathcal{C}_{2}}$ between 285 the multiple APs and UEs within $C_{2}$ as:

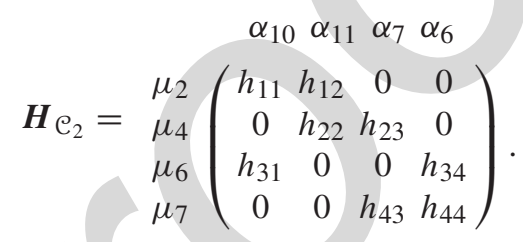

In order to attain mutually interference-free signals at the 287 receivers, the transmitted signals $\boldsymbol{X}_{\mathcal{C}_{2}}=\left[x_{1}, x_{2}, x_{3}, x_{4}\right]$ are 288 precoded as $\left(\boldsymbol{P}_{\mathcal{C}_{2}} \cdot \boldsymbol{X}_{\mathcal{C}_{2}}\right)$ and we may write $\boldsymbol{P}_{\mathcal{C}_{2}}=\left(\boldsymbol{G}_{\mathcal{C}_{2}} \cdot 289\right.$ $\left.\boldsymbol{\Omega}_{\mathcal{C}_{2}}\right)$, where the matrix $\boldsymbol{G}_{\mathcal{C}_{2}}=\boldsymbol{H}_{\mathcal{C}_{2}}^{H} \cdot\left(\boldsymbol{H}_{\mathcal{C}_{2}} \cdot \boldsymbol{H}_{\mathcal{C}_{2}}^{H^{2}}\right)^{-1}$ obeys 290 the $\mathrm{ZF}$ criterion for the sake of obtaining an interference-free 291 identity matrix for $\boldsymbol{H}_{\mathcal{C}_{2}} \cdot \boldsymbol{G}_{\mathcal{C}_{2}}=\boldsymbol{I}_{4}$ and $\boldsymbol{\Omega}_{\mathcal{C}_{2}}$ is introduced in 292 order to satisfy the power constraint. Hence, the ICI can be 293 totally eliminated at the multiple AP transmitters and as a result, 294 all the UEs receive mutually interference-free signals. Let us 295 now elaborate on the VT techniques a little further in general 296 terms and derive the formations of $\boldsymbol{G}$ and $\boldsymbol{\Omega}$.

Each UC-VT cluster $\mathcal{C}_{n}$ is constituted by a set of APs 298 $\mathcal{V}_{A, \mathcal{C}_{n}}$ with a cardinality of $N_{A, \mathcal{C}_{n}}$ and a set of UEs $\mathcal{V}_{U, \mathcal{C}_{n}} 299$ with a cardinality of $N_{U, \mathcal{C}_{n}}$. Let further $\boldsymbol{X}_{t} \in \mathbb{R}^{N_{U, \mathcal{C}_{n}} \times 1}$ and 300 $\boldsymbol{Y}_{r} \in \mathbb{R}^{N_{U, e_{n} \times 1}}$ denote the vectors of transmitted and received 301 signals, respectively. Upon using VT, we have

$$
\boldsymbol{Y}_{r}=\gamma \cdot P_{t} \cdot \boldsymbol{H} \cdot \boldsymbol{G} \cdot \boldsymbol{\Omega} \cdot \boldsymbol{X}_{t}+\boldsymbol{N},
$$

where $\gamma$ and $P_{t}$ denote the Optical/Electronic (O/E) conver- 303 sion efficiency and the transmitted optical power, respectively. 304 
Furthermore, $\boldsymbol{N}$ denotes the noise, while the channel-matrix $\boldsymbol{H} \in \mathbb{R}^{N_{U, e_{n}} \times N_{A, \mathrm{e}_{n}}}$ hosts the DC attenuations between the $N_{U, \mathcal{C}_{n}}$ UEs and the $N_{A, \mathfrak{C}_{n}}$ APs, while the matrix $\boldsymbol{G}=\boldsymbol{H}^{H}$. $\left(\boldsymbol{H} \cdot \boldsymbol{H}^{H}\right)^{-1}$ obeys the ZF criterion, which hence results in a beneficial interference-free identity matrix for $\boldsymbol{H} \cdot \boldsymbol{G}=\boldsymbol{I}_{N_{U, e_{n}}}$. Finally, the matrix $\boldsymbol{\Omega}$ is introduced to enforce the per-AP power constraints, hence we have

$$
\boldsymbol{\Omega}=\varphi \boldsymbol{I}_{N_{U, \mathrm{e}_{n}}}, \quad \varphi=\min _{i=1,2, \ldots, N_{A, \mathfrak{e}_{n}}} \sqrt{\frac{1}{\|\boldsymbol{G}(i,:)\|_{F}^{2}}},
$$

where $\boldsymbol{G}(i,:)$ is the $i$ th row of $\boldsymbol{G}$. To elaborate a little further, assuming that we have the per-AP optical power constraint of $P_{t}$, the signal transmitted with the equal power from the $i$ th $\mathrm{AP}$ is $\varphi^{2}\|\boldsymbol{G}(i,:)\|_{F}^{2}$. Note that we have $P_{e}=$ $\pi P_{t}^{2}$, when considering the Asymmetrically-Clipped Optical OFDM (ACO-OFDM) [8]. Hence, we have $\varphi^{2}\|\boldsymbol{G}(i,:)\|_{F}^{2} \leq$ $\pi P_{t}^{2} \Rightarrow \varphi \leq \pi P_{t}^{2} / \sqrt{\|\boldsymbol{G}(i,:)\|_{F}^{2}}$. In order to let each AP satisfy the power constraint, we have $\varphi=\min _{i} \pi P_{t}^{2} / \sqrt{\|\boldsymbol{G}(i,:)\|_{F}^{2}}$, as indicated in (7). Furthermore, let us define the SINR as the aggregate electronic power over the noise power in a bandwidth of $B$ [MHz] [7] plus the sum of the electronic power received from other optical sources in the vicinity. Since the corresponding electronic power is proportional to the square of the electronic current's amplitude and both the intra-cluster and inter-cluster LOS interferences are mitigated, we may express the SINR for a particular UE $\mu_{j}$ within the cluster $\mathcal{C}_{n}$ as

$$
\xi=\frac{\gamma^{2} P_{t}^{2} \varphi^{2} \pi}{N_{0} B+I_{r}}
$$

where $I_{r}$ is the interference imposed by the reflected light. Since the interference power received by the cluster under consideration is influenced by the ZF-based VT within other clusters, for simplicity, we assume that the interference imposed is always equal to its maximum value, which characterizes the worst-case situation in our VT cluster formations. Furthermore, $N_{0}\left[\mathrm{~A}^{2} / \mathrm{Hz}\right]$ is the noise power spectral density dominated by the shot noise $N_{\text {shot }}[10]$ given by $N_{0} \cong N_{\text {shot }}=q I_{a}\left(P_{r}\right) \sim$ $10^{-22}$, where $q$ denotes the electron charge and $I_{a}\left(P_{r}\right)$ is the photo-current at the receiver [7].

Note that there are two popular techniques of constructing white LEDs, namely either by mixing the Red-Green-Blue (RGB) frequencies using three chips, or by using a single blue LED chip with a phosphor layer. We consider the latter one, which is the favoured commercial version. Although the terminology of 'white' LED gives the impression of having all frequency components across the entire visible light spectrum, in fact only the blue frequency-range is detected. More explicitly, not even the entire blue frequency-range is detected, since the less responsive phosphorescent portion of the frequencyband is ignored. Hence, the modulation bandwidth is typically around $20 \mathrm{MHz}$, albeit this measured bandwidth depends on the specific LED product used. Given this $20 \mathrm{MHz}$ bandwidth, we are now ready to employ ACO/DC biased Optical (DCO)-OFDM and partition it into arbitrary frequency reuse patterns.

\section{Methodology}

Let us now schedule multiple UEs in the VLC system in a 355 PF manner by taking into account our UC-VT cluster forma- 356 tion, which is ultimately a joint UC-VT cluster formation and 357 MUS problem. In this section, we commence with a general for- 358 mulation of this joint problem and then propose an exhaustive 359 search method, which finds the optimal solution maximizing 360 the aggregate utility of the VLC system considered. In order 361 to reduce the computational complexity imposed, the original 362 problem is reformulated as an MWM problem, whose optimal 363 solution is provided by the classic KM-algorithm-based [24] 364 approach. For further simplifying the MUS process, we pro- 365 pose a greedy scheduling algorithm for finding a suboptimal 366 solution for our original joint problem, whilst imposing a sig- 367 nificantly reduced complexity. Note that for simplicity, we only 368 consider LOS links in terms of constructing UC-VT clusters. 369 By contrast, in addition to the LOS component, the effect of the 370 first reflection will also be considered, when calculating both 371 the UEs' SINR and the achievable data rate, as indicated in (8). 372 However, our algorithm is a generic one, which may be readily 373 applied, when considering the reflected light for UC-VT cluster 374 formation.

\section{A. Problem Formulation}

Our goal is to find the optimal UC-VT cluster formation for 377 maximizing the long-term network-wide utility, while schedul- 378 ing UEs in a PF manner, which is ultimately a joint cluster 379 formation and MUS problem. In order to implement a PF 380 scheduler, the weight of each link between APs and UEs may 381 be defined as

$$
\omega\left(e_{\alpha_{i}, \mu_{j}}\right)=\frac{r_{\alpha_{i}, \mu_{j}}}{\hat{r}_{\mu_{j}}}, \quad e_{\alpha_{i}, \mu_{j}} \in \mathcal{E},
$$

where $r_{\alpha_{i}, \mu_{j}}$ denotes the achievable data rate of the UE $\mu_{j}$ from 383 the AP $\alpha_{i}$ during the current slot. Since the SINR $\xi$ experienced 384 by a particular UE is determined by the channel attenuation 385 matrix (5) between the APs and UEs within the cluster, $r_{\alpha_{i}, \mu_{j}} 386$ should be a function of the cluster formation, which may be 387 written as:

$$
r_{\alpha_{i}, \mu_{j}}=f\left(\mathcal{E}^{\prime}\right), \quad e_{\alpha_{i}, \mu_{j}} \in \mathcal{E}^{\prime}, \mathcal{E}^{\prime} \subseteq \mathcal{E},
$$

where $\mathcal{E}^{\prime}$ is the set of established links, after the UEs have 389 been scheduled and the UC-VT clusters have been constructed. 390 Furthermore, $\hat{r}_{\mu_{j}}$ denotes the long-term average throughput of 391 the UE $\mu_{j}$, which may be obtained over a time window $T_{F}$ as a 392 moving average according to [30]:

$$
\hat{r}_{\mu_{j}}^{(t)}= \begin{cases}\left(1-\frac{1}{T_{\mathrm{F}}}\right) \hat{r}_{\mu_{j}}^{(t-1)}+\frac{1}{T_{\mathrm{F}}} r_{\alpha_{i}, \mu_{j}}^{(t)}, & \text { if scheduled, } \\ \left(1-\frac{1}{T_{\mathrm{F}}}\right) \hat{r}_{\mu_{j}}^{(t-1)}, & \text { if not scheduled. }\end{cases}
$$

For a given UC-VT cluster formation $\left\{\mathcal{C}_{n}\right\}$, the aggregate util- 394 ity may be formulated by taking into account the weight of 395 
each edge, where again, the weight physically represents the PF scheduling priority of the link [30], which is formulated as:

$$
\begin{aligned}
W & =\sum_{e_{\alpha_{i}, \mu_{j}} \in \mathcal{E}^{\prime}} \omega\left(e_{\alpha_{i}, \mu_{j}}\right) \\
& =\sum_{\alpha_{i} \in \mathcal{V}_{A}^{\prime}} \sum_{\mu_{j} \in \mathcal{V}_{U}^{\prime}} \frac{r_{\alpha_{i}, \mu_{j}}}{\hat{r}_{\mu_{j}}}, \quad \mathcal{E}^{\prime} \subseteq \mathcal{E},
\end{aligned}
$$

where $\mathcal{V}_{A}^{\prime}$ and $\mathcal{V}_{U}^{\prime}$ denote the serving APs and the scheduled UEs set, respectively. It is plausible that various UC-VT cluster formations may lead to different total utility. The maximum value of the aggregate utility $W$ may be achieved by finding the optimal cluster formation. Thus, our problem may be described as selecting an appropriate set of edges $\mathcal{E}^{*}$ from $\mathcal{E}$ and then forming several UC-VT clusters, which maximizes (12). Hence, our Objective Function (OF) may be formulated as:

$$
\mathcal{E}^{*}=\arg \max _{\mathcal{E}^{\prime} \subseteq \mathcal{E}}(W)=\arg \max _{\mathcal{E}^{\prime} \subseteq \mathcal{E}}\left(\sum_{\alpha_{i} \in \mathcal{V}_{A}^{\prime}} \sum_{\mu_{j} \in \mathcal{V}_{U}^{\prime}} \frac{r_{\alpha_{i}, \mu_{j}}}{\hat{r}_{\mu_{j}}}\right) .
$$

Note that in (13) we focus our attention on the aggregate utility of the entire system and do not distinguish, which particular APs and UEs belong to which UC-VT clusters. Let us now discuss the constraint of (13), from the perspective of a single UC-VT cluster. As mentioned in Section II-B, the number of scheduled UEs should not exceed the service capability of a cluster employing VT, where again, the maximum number of UEs supported is equal to the number of APs. Hence, within a single UC-VT cluster $C_{n}$ we have

$$
N_{A, \mathcal{C}_{n}} \geq N_{U, \mathcal{C}_{n}} .
$$

For solving (13) under the constraint of (14) and finding the optimal cluster formation, we have to know the weight of all edges in $\mathcal{E}$. However, according to (9), the weight $\omega\left(e_{\alpha_{i}, \mu_{j}}\right)$ of a particular link is defined as a function of the data rate achieved by one of its endpoints $\mu_{j}$ during its reception from the other endpoint $\alpha_{i}$, which can only be determined after all clusters have been formed, as briefly introduced in Section II-C. To the best of our knowledge, the optimal solution of this joint problem may only be found via exhaustive search.

\section{B. Optimization of the Joint Problem}

Given a VLC network topology having $N_{A}$ optical APs and $N_{U}$ UEs, it may be composed of some independent components, for example as shown in Fig. 2b. Note that these naturally disjoint components of the network may not constitute the final formations of the UC-VT cluster. More explicitly, there is no limitation concerning the number of APs and UEs within each single component of the network, apart from the fact that within a UC-VT cluster the cardinality of the actively served UE vertex set should be no larger than that of the AP set, as indicated by (14). Each UC-VT cluster should be an independent component of the network, where no ICI is imposed on the neighbouring clusters. Furthermore, each individual network component should be connected at the outset, but each may become disconnected and partitioned into several sub-components/clusters throughout the process of scheduling the UEs, as shown in 439 Fig. 2 d, where $\mathcal{S}_{2,1}, \mathcal{S}_{2,2}$ and $\mathcal{S}_{2,3}$ will be regarded as a large 440 merged cluster.

Still referring to Fig. 2b, in order to find the optimal cluster 442 formation for maximizing (12), the optimization is performed 443 separately in $\mathcal{Q}_{1}, \mathcal{Q}_{2}$ and $\mathcal{Q}_{3}$, which are independent network 444 components. Within $\mathcal{Q}_{1}$, only a single UE $\mu_{1}$ is capable of con- 445 necting with the AP $\alpha_{4}$, where $\alpha_{4}$ either supports $\mu_{1}$ or it will be 446 turned off. Therefore, there are two AP-UE combination scenar- 447 ios for $\mathcal{Q}_{1}$. Within $\mathcal{Q}_{2}$, there are three UEs, i.e. $\mu_{2}, \mu_{3}$ and $\mu_{6}, 448$ which are within the coverage of the AP $\alpha_{10}$. Hence, $\alpha_{10}$ may 449 either select one of them to support or become inactive. Thus, 450 there are $(3+1)$ choices for $\alpha_{10}$. Similarly, the other APs $\alpha_{11}, 451$ $\alpha_{7}$ and $\alpha_{6}$ have $(2+1),(3+1)$ and $(3+1)$ choices, respec- 452 tively. Therefore, the number of possible AP-UE combinations 453 within $Q_{2}$ is $(4 \times 3 \times 4 \times 4=192)$. $Q_{3}$ has an easier situation, 454 where the AP $\alpha_{16}$ may either select one UE from $\left\{\mu_{9}, \mu_{10}\right\} \quad 455$ or opts for providing no services. For the entire network of 456 Fig. 2b, the number of possible AP-UE combinations becomes 457 $((2-1)+(192-1)+(3-1)=194)$. Finally, we take into 458 account the undesired scenario, where all APs are out of service 459 by subtracting 1 . Generally speaking, our exhaustive search- 460 based approach of finding the optimal UC-VT cluster formation 461 is detailed below.

i) For each separate network component $Q_{m}$ relying on 463 $N_{A, Q_{m}}$ APs and $N_{U, Q_{m}}$ UEs, let $N_{U, Q_{m}}^{\alpha_{i}}$ denote the num- 464 ber of possible links between a certain AP $\alpha_{i}$ with the UEs 465 within its coverage, where $i=1,2, \ldots, N_{A, \Omega_{m}}$. 466

ii) Note that not the entire set of APs has to be active during 467 the scheduling process. In other words, we do not limit the 468 number of active APs or scheduled UEs, when aiming for 469 finding the optimal cluster formation. Thus the concept 470 of a virtual link is introduced for each AP, which theo- 471 retically exists, but it is turned off. Hence, the number of 472 possible AP-UE combinations in $\mathcal{Q}_{m}$ may be expressed as 473

$$
\prod_{i=1}^{N_{A, Q_{m}}}\left(N_{U, Q_{m}}^{\alpha_{i}}+1\right)-1
$$

where we have $1 \leq N_{U, Q_{m}}^{\alpha_{i}} \leq N_{U, Q_{m}}$. Note that in (15), 474 subtracting 1 implies that we have removed the undesired 475 scenario, where all APs are turned off. 476

iii) For each possible UC-VT cluster formation in $Q_{m}$, the 477 aggregate utility can be calculated and the optimal for- 478 mation associated with the maximum utility is found 479 correspondingly. Since each network component $\mathcal{Q}_{m}$ is 480 independent, the optimal cluster formation is separately 481 found in each of them. Hence, for finding the optimal 482 solution of (13) for the entire system, we need to repeat 483 the process of ii) in each $Q_{m}$. Thus the total number of 484 possible AP-UE combinations is the summation of (15) 485 for each $2_{m}$, which may be expressed as

$$
\sum_{m}\left(\prod_{i=1}^{N_{A, Q_{m}}}\left(N_{U, Q_{m}}^{\alpha_{i}}+1\right)-1\right)
$$

The number of all possible cluster formations within a single 487 scheduling time slot at a ms-based scale is given by (16), which 488 
is jointly determined by the number of APs $\left(N_{A}, Q_{m}\right)$ and number of UEs $\left(N_{U, Q_{m}}\right)$ as well as by the specific distribution of the UEs $\left(N_{U, Q_{m}}^{\alpha_{i}}\right)$. For a network associated with a low density of UEs and a small number of APs, a desirable cluster formation solution may be achieved by exhaustively searching all the possibilities. For example, when 16 APs support 10 UEs, the optimal association will be found after searching $\sim 10^{4}$ possible cluster formations. However, this search-space may become excessive within a time slot at a ms-based scale even for a modest-scale network, which makes the exhaustive search strategy unacceptable owing to its computational complexity. For example, as many as $\sim 10^{7}$ cluster formations have to be searched within a single processing time slot, when there are 20 UEs supported by 16 APs. Hence, instead of solving the joint problem directly, we update the definition of the weight for each link and reformulate the original problem with the goal of significantly reducing the complexity, as it will be detailed in Section IV.

\section{Distance-based Weight and Problem Reformulation}

In (9), the weight of each link is related to the UE's achievable data rate, which cannot be determined before the UC-VT clusters have finally been constructed. Our ultimate goal is that of finding the optimal cluster formation based on the sum weight attained by appropriately scheduling the UEs, as indicated by (13). In other words, the cluster formation and MUS problems were originally coupled. Hence, we opt for simplifying the original problem by adopting a deterministic weight for each AP-UE link. Thus, the maximization of the sum weight may be realized before the UC-VT clusters are constructed, and as a benefit, the joint cluster formation and MUS problem becomes decoupled.

As mentioned in Section III-B, the weight of each link between the AP and the UE is non-deterministic, which is influenced by how the UC-VT clusters are constructed, while the optimal cluster formation solution is determined by maximizing the sum weight of all the scheduled links. Hence, we opt for bypassing the non-deterministic weight assignment and instead, we opt for selecting active links according to their optical channel quality, which is significantly affected by the UE's position, according to (1). We directly adopt each UE's position information for determining the weight of each link and introduce a new weighted bipartite graph $\mathcal{G}_{d}(\mathcal{V}, \mathcal{E})$, which is constructed based on the original graph $\mathcal{G}(\mathcal{V}, \mathcal{E})$ and they have the same vertex and edge sets. However, the weight of each edge is redefined as

$$
\omega_{d}\left(e_{\alpha_{i}, \mu_{j}}\right)=\frac{1 / l_{\alpha_{i}, \mu_{j}}^{3}}{\hat{r}_{\mu_{j}}}, \quad e_{\alpha_{i}, \mu_{j}} \in \mathcal{E},
$$

where $l_{\alpha_{i}, \mu_{j}}$ represents the distance between the AP $\alpha_{i}$ and the $\mathrm{UE} \mu_{j}$. Given that the APs are fixed, the weight is determined by the specific position of each $\mathrm{UE} \mu_{j}$. It can be readily seen from (1) that the VLC links having a shorter length have a better channel quality. Therefore, the weight is inversely proportional to the distance and thus the links associated with better channels have a higher weight. Note that if the UE $\mu_{j}$ is too far away from the AP $\alpha_{i}$, namely $\mu_{j}$ is not within the coverage of $\alpha_{i}$, it is reasonable to assume having $\omega_{d}\left(e_{\alpha_{i}, \mu_{j}}\right)=0$.
Our problem becomes that of selecting a subset of links $\mathcal{E}_{d}^{*} 542$ having a better channel quality, and along with their endpoints 543 they represent our UC-VT cluster formation. In general, within 544 a UC-VT cluster, multiple APs serve multiple UEs and there 545 may not be a one-to-one relationship. Nonetheless, in the first 546 MUS step, we could select the one-to-one AP-UE pairs accord- 547 ing to their distance-based weight, where the serving APs and 548 the scheduled UEs are determined. Then, in the cluster for- 549 mation step, the cluster may be constructed by adding other 550 possible links between the selected AP-UE set. Thus the MUS 551 and cluster formation problem is decoupled and solved sepa- 552 rately. Note that in the MUS step, a specific set of the links 553 between all the AP-UE pairs, which do not share the same AP 554 or UE, is said to represent independent edges and they con- 555 stitute a matching $\mathcal{M}$ defined over the graph. For example, in 556 Fig. 2b we have $6 \mathrm{AP}$ vertices plus $10 \mathrm{UE}$ vertices as well as 557 14 edges. In order to construct a matching, 6 UEs are selected 558 and each of them matches a specific AP associated with one 559 edge, e.g. $\left\{\alpha_{4} \rightarrow \mu_{1}, \alpha_{10} \rightarrow \mu_{3}, \alpha_{11} \rightarrow \mu_{4}, \alpha_{7} \rightarrow \mu_{7}, \alpha_{6} \rightarrow 560\right.$ $\left.\mu_{6}, \alpha_{16} \rightarrow \mu_{9}\right\}$. Furthermore, we have $\mathcal{M} \subseteq \mathcal{E}_{d}^{*} \subseteq \mathcal{E}$. To elabo- 561 rate a litter further in general terms, let us first formally define 562 the matching over a graph. As mentioned in Section III-B, the 563 network graph model may be disconnected and divided into 564 multiple independent components. For an individual compo- 565 nent, denoted by $\mathcal{Q}_{m}\left(\mathcal{V}_{\mathcal{Q}_{m}}, \mathcal{E}_{\mathcal{Q}_{m}}\right)$, which is a subgraph of $\mathcal{G}_{d} 566$ associated with the vertex set $\mathcal{V}_{Q_{m}}$ and the edge set $\mathcal{E}_{\mathcal{Q}_{m}}$, a 567 matching $\mathcal{M}_{Q_{m}}$ may be defined as a specific subset of the edge 568 set $\mathcal{E}_{Q_{m}}$, where no pair of edges shares a vertex within $\mathcal{M}_{Q_{m}}$. 569 It is plausible that the cardinality of the edge-subset $\mathcal{M}_{Q_{m}}$ is 570 given by the number of the $\mathcal{M}_{Q_{m}}$-saturated AP/UE vertices, 571 which belongs to the edges of $\mathcal{M}_{Q_{m}}$. Otherwise, the vertices not 572 belonging to the edges of $\mathcal{M}_{Q_{m}}$ are said to be $\mathcal{M}_{Q_{m}}$-unsaturated. 573 Hence, if we allow as many UEs as possible to be scheduled, $\mathcal{M} 574$ should have the highest possible cardinality. Furthermore, con- 575 sidering the weight of each edge, our cluster formation problem 576 may be further reformulated as a MWM problem, where the OF 577 may be written as:

$$
\begin{aligned}
\mathcal{M}_{Q_{m}}^{*} & =\arg \max _{\mathcal{M}_{Q_{m}}}\left(W_{Q_{m}}\right) \\
& =\arg \max _{\mathcal{M}_{Q_{m}}}\left(\sum_{\alpha_{i} \in \mathcal{V}_{Q_{m}}, \mu_{j} \in \mathcal{V}_{Q_{m}}} \omega_{d}\left(e_{\alpha_{i}, \mu_{j}}\right)\right) .
\end{aligned}
$$

Upon solving (18) within each individual network compo- 579 nent, a set of APs as well as UEs is selected in order to form 580 a UC-VT cluster along with all links between them. Thus, the 581 solution of the MWM problem is expected to provide a sub- 582 optimal result for our original joint MUS and UC-VT cluster 583 formation problem, which is however found at a significantly 584 reduced complexity.

\section{Optimal MWM}

If we construct a $\left(N_{A, Q_{m}} \times N_{U, Q_{m}}\right)$-element weight matrix 587 $\left(\omega_{d}\left(e_{\alpha_{i}, \mu_{j}}\right)\right)$ for each of the individual component $Q_{m}$, the prob- 588 lem of (18) may be viewed as being equivalent to finding a set 589 of independent elements from $\left(\omega_{d}\left(e_{\alpha_{i}, \mu_{j}}\right)\right)$, in order to maxi- 590 mize the sum of these elements. The definition of independent 591 


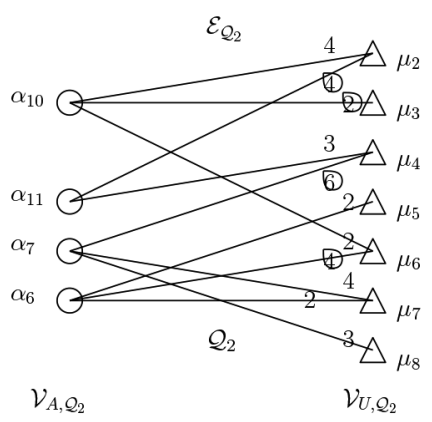

(a)

\begin{tabular}{|c|c|c|c|c||c|c|c|c|c|}
\hline$\alpha_{10}$ & $\alpha_{11}$ & $\alpha_{7}$ & $\alpha_{6}$ & $W_{Q}$ & $\alpha_{10}$ & $\alpha_{11}$ & $\alpha_{7}$ & $\alpha_{6}$ & $W_{Q}$ \\
\hline \hline$\mu_{2}$ & $\mu_{4}$ & $\mu_{7}$ & $\mu_{5}$ & 13 & $\mu_{3}$ & $\mu_{4}$ & $\mu_{7}$ & $\mu_{5}$ & 11 \\
\hline$\mu_{2}$ & $\mu_{4}$ & $\mu_{7}$ & $\mu_{6}$ & 15 & $\mu_{3}$ & $\mu_{4}$ & $\mu_{7}$ & $\mu_{6}$ & 13 \\
\hline$\mu_{2}$ & $\mu_{4}$ & $\mu_{8}$ & $\mu_{5}$ & 12 & $\mu_{3}$ & $\mu_{4}$ & $\mu_{8}$ & $\mu_{5}$ & 10 \\
\hline$\mu_{2}$ & $\mu_{4}$ & $\mu_{8}$ & $\mu_{6}$ & 14 & $\mu_{3}$ & $\mu_{4}$ & $\mu_{8}$ & $\mu_{6}$ & 12 \\
\hline$\mu_{2}$ & $\mu_{4}$ & $\mu_{8}$ & $\mu_{7}$ & 12 & $\mu_{3}$ & $\mu_{4}$ & $\mu_{8}$ & $\mu_{7}$ & 10 \\
\hline$\mu_{3}$ & $\mu_{2}$ & $\mu_{4}$ & $\mu_{5}$ & 14 & $\mu_{6}$ & $\mu_{2}$ & $\mu_{4}$ & $\mu_{5}$ & 14 \\
\hline$\mu_{3}$ & $\mu_{2}$ & $\mu_{4}$ & $\mu_{6}$ & 16 & $\mu_{6}$ & $\mu_{2}$ & $\mu_{4}$ & $\mu_{7}$ & 14 \\
\hline$\mu_{3}$ & $\mu_{2}$ & $\mu_{4}$ & $\mu_{7}$ & 15 & $\mu_{6}$ & $\mu_{2}$ & $\mu_{7}$ & $\mu_{5}$ & 12 \\
\hline$\mu_{3}$ & $\mu_{2}$ & $\mu_{7}$ & $\mu_{5}$ & 12 & $\mu_{6}$ & $\mu_{2}$ & $\mu_{8}$ & $\mu_{5}$ & 11 \\
\hline$\mu_{3}$ & $\mu_{2}$ & $\mu_{7}$ & $\mu_{6}$ & 14 & $\mu_{6}$ & $\mu_{2}$ & $\mu_{8}$ & $\mu_{7}$ & 11 \\
\hline$\mu_{3}$ & $\mu_{2}$ & $\mu_{8}$ & $\mu_{5}$ & 11 & $\mu_{6}$ & $\mu_{4}$ & $\mu_{7}$ & $\mu_{5}$ & 11 \\
\hline$\mu_{3}$ & $\mu_{2}$ & $\mu_{8}$ & $\mu_{6}$ & 13 & $\mu_{6}$ & $\mu_{4}$ & $\mu_{8}$ & $\mu_{5}$ & 10 \\
\hline$\mu_{3}$ & $\mu_{2}$ & $\mu_{8}$ & $\mu_{7}$ & 11 & $\mu_{6}$ & $\mu_{4}$ & $\mu_{8}$ & $\mu_{7}$ & 10 \\
\hline
\end{tabular}

(b)

Fig. 3. (a) A component of $\mathcal{G}_{d}, Q_{2}$, where the distance-based weight of each link is assumed to be as seen in (a), which is inversely proportional to the AP-UE distances in Fig. 1a with the UEs' being randomly distributed. (b) List of all possible AP-UE matchings in $Q_{2}$ and the corresponding sum weight $W_{Q}$. The best matching associated with the circled weights of (a) is the one in the grey-shaded line 7.

elements indicates that none of them occupies the same row or column, where a row represents an AP and a column represents a UE. To be more explicit, the selected set of the independent elements in the weight matrix corresponds to a matching of the graph, since a single element represents an edge of the graph and no pair of these elements shares the same AP or UE. Thus our MWM problem has also been interpreted in a matrix form. Before finding the optimal solution of the afore-mentioned MWM problem, let us first introduce Theorem 1.

Theorem 1: Given the $\left(n_{r} \times n_{r}\right)$-element matrix $\left(a_{i j}\right)$ and $\left(b_{i j}\right)$, as well as the column vector $\left(c_{i}\right)$ and the row vector $\left(r_{j}\right)$, satisfying $b_{i j}=c_{i}+r_{j}-a_{i j}$, provided the permutation $p\left(p_{i}\right.$ : $\left.i=1, \ldots, n_{r}\right)$ of the integers $1, \ldots, n_{r}$ minimizes $\sum_{i=1}^{n_{r}} a_{i p_{i}}$, $p$ then also maximizes $\sum_{i=1}^{n_{r}} b_{i p_{i}}$.

Proof: Let $p$ be a permutation of the integers $1,2, \ldots, n$ minimizing $\sum_{i=1}^{n_{r}} a_{i p_{i}}$, then we have

$$
\sum_{i=1}^{n_{r}} b_{i p_{i}}=\sum_{i=1}^{n_{r}} c_{i}+\sum_{i=1}^{n_{r}} r_{p_{i}}-\sum_{i=1}^{n_{r}} a_{i p_{i}}
$$

Since the first two terms are constant and independent of $p, \sum_{i=1}^{n_{r}} b_{i p_{i}}$ is maximized, when $\sum_{i=1}^{n_{r}} a_{i p_{i}}$ is minimized by $p$.

Hence, if we want to find the optimal assignment solution for maximizing $\sum_{i=1}^{n} b_{i, p_{i}}$, what we have to do is to transform $\left(b_{i j}\right)$ into $\left(a_{i j}\right)$ as mentioned above and then find the optimal solution minimizing $\sum_{i=1}^{n} a_{i, p_{i}}$, where $\left(a_{i j}\right)$ and $\left(b_{i j}\right)$ are said to be equivalent. For a rectangular $\left(n_{r} \times n_{c}\right)$-element matrix $\left(a_{i j}^{\prime}\right)$, we can obtain a square matrix $\left(a_{i j}\right)$ by attaching $\mid n_{r}-$ $n_{c} \mid$ lines of zero elements to $\left(a_{i j}^{\prime}\right)$. Thus, $\left(a_{i j}^{\prime}\right)$ and $\left(a_{i j}\right)$ have the same optimal assignment solution and Theorem 1 can be readily applied for non-square rectangular matrices, where we have $n_{r} \neq n_{c}$.

In order to solve our MWM problem, which is derived from our joint cluster formation and MUS problem, we introduce the classic Kuhn-Munkres (KM) algorithm [24], [25], which is an efficient method of solving the matching problems of bipartite graphs and may be readily applied in a symmetric graph. However, the number of VLC UEs is usually higher than that of the optical APs within a single network component $2_{m}$, which results in an asymmetric bipartite graph. Owing to the efforts of
Bourgeois and Lassalle [26], an extension of the KM algorithm 627 was developed for non-square rectangular matrices. Relying on 628 this approach, we introduce a KM-algorithm-based technique 629 of solving our UC-VT cluster formation problem. The mathe- 630 matical formulation of the extended KM algorithm of [26] may 631 be described as that of finding a set of $k$ independent elements 632 $k=\min \left\{n_{r}, n_{c}\right\}$ from a given $\left(n_{r} \times n_{c}\right)$-element matrix $\left(b_{i, j}\right), 633$ in order to minimize the sum of these elements. However, our 634 problem is not a minimization, but a maximization problem 635 associated with the OF of (18). Therefore, we first transform our 636 MWM problem into an equivalent assignment problem based 637 upon Theorem 1 and then invoke the KM algorithm for finding 638 the optimal solution of the equivalent problem, which is also 639 optimal for our MWM problem. Furthermore, since the MWM 640 result of each naturally disjoint network component is mutually 641 independent, the matching algorithm is executed within each 642 individual component in a parallel manner.

As shown in Fig. 3a, $2_{2}$ is an independent network compo- 644 nent and also a subgraph of our weighted graph $\mathcal{G}_{d}$, which also 645 shows the individual weights of the $\left\{\alpha_{i}-\mu_{j}\right\}$ links. In order 646 to schedule the maximum number of UEs, given the four APs 647 in $2_{2}$, four of them will be selected and each one is paired 648 with a specific AP, where the possible matchings and the corre- 649 sponding sum weight values are shown in Fig. 3b. For example, 650 bearing in mind Fig. 3a, the first matching of the first row in 651 Fig. 3b may represent $\left\{\alpha_{10} \rightarrow \mu_{2}, \alpha_{11} \rightarrow \mu_{4}, \alpha_{7} \rightarrow \mu_{7}, \alpha_{6} \rightarrow 652\right.$ $\left.\mu_{5}\right\}$, which leads to a sum weight of $W_{Q}=\sum_{l=1}^{4} \omega_{l}=4+653$ $3+4+2=13$. The specific matching of the seventh row in 654 Fig. $3 \mathrm{~b}$ is $\left\{\alpha_{10} \rightarrow \mu_{3}, \alpha_{11} \rightarrow \mu_{2}, \alpha_{7} \rightarrow \mu_{4}, \alpha_{6} \rightarrow \mu_{6}\right\}$, which 655 is represented by the shaded row of Fig. 3b. This achieves the 656 largest sum weight of $W_{Q}=\sum_{l=1}^{4} \omega_{l}=2+4+6+4=16.657$ The corresponding weights in Fig. $3 \mathrm{a}$ are circled. Hence they 658 represent the optimal matching in the scenario considered. 659

Instead of listing all matchings, we now proceed by con- 660 structing an equivalent minimization problem for our MWM 661 and invoke the KM algorithm [24], [26] for finding the opti- 662 mal solution, which is described in detail in Appendix A. 663 As shown in Fig. 4a, the KM-algorithm-based approach pro- 664 vides the optimal solution for the MWM problem (18), with 665 its UE-AP distance-based weight defined by (17). The matched 666 AP-UE pairs form a UC-VT cluster and the aggregate util- 667 ity in (12) can be calculated according to the matching result. 668 


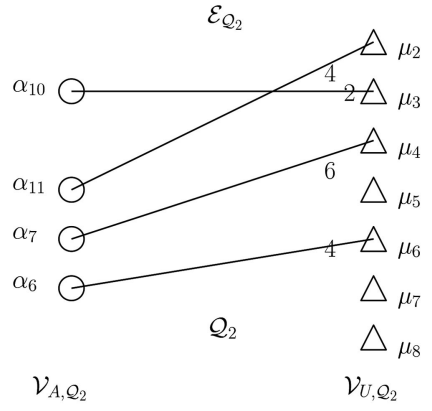

(a)

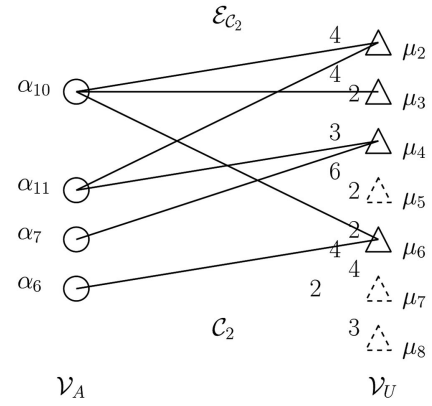

(b)

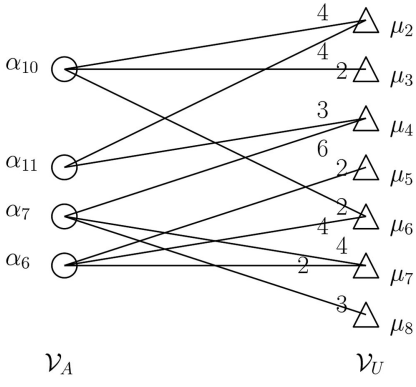

(a)

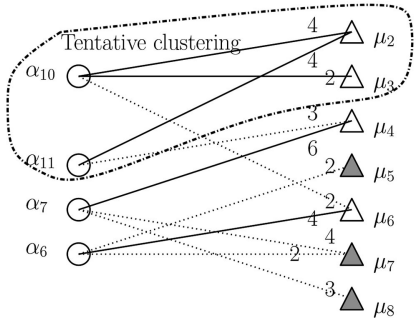

$\mathcal{V}_{A}$

(c)

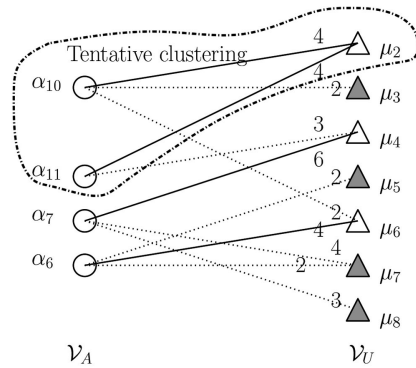

(b)

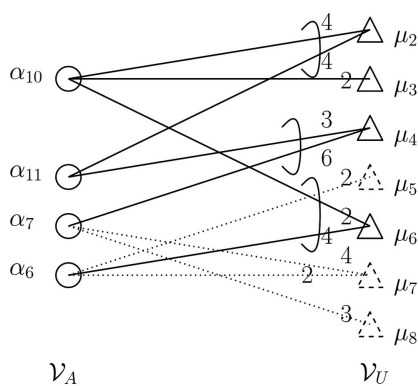

(d)
Fig. 4. (a) The optimal solution for the MWM problem (18) relying on the distance-based weight defined by (17), which is provided by the KM-algorithmbased assignment. (b) The UC-VT cluster formation based on the matching result of Fig. 3a, where more links are added for employing VT and thus the multiple APs $\left\{\alpha_{10}, \alpha_{11}, \alpha_{7}, \alpha_{6}\right\}$ are capable of supporting all the scheduled UEs $\left\{\mu_{2}, \mu_{3}, \mu_{4}, \mu_{6}\right\}$ simultaneously. The triangle with a dashed boundary denotes the specific UE, which is not scheduled during the current slot.

However, by employing VT among the set of APs and UEs, the actual cluster may be formed with the aid of more links, as seen in Fig. 4b. Thus, the UC-VT cluster formation provided by the single-to-single matching solution may not be optimal for (13), but it is capable of offering an acceptable suboptimal solution attained at a lower complexity than that of the exhaustive search. Explicitly, it has a complexity order of $O\left(k^{2} \times l\right)$ [26], where we have $k=\min \left\{N_{A, Q_{m}}, N_{U, Q_{m}}\right\}$ and $l=\max \left\{N_{A, Q_{m}}, N_{U, Q_{m}}\right\}$. The complexity of both the exhaustive search and KM algorithm will be investigated in Section IV in the context of our VLC-based network.

\section{E. Proposed Greedy Cluster Formation/MUS Algorithm}

In order to further simplify the procedures of scheduling the UEs in our UC-VT cluster formation, in this section we propose a greedy cluster formation/MUS algorithm operating at a low complexity, which is also capable of achieving a near-optimal solution for our original cluster formation problem of (13). Before discussing our proposed MUS problem, let us first introduce some notations. Explicitly, $\nu_{U, \alpha_{i}}$ denotes the set of UEs within the coverage of a specific AP $\alpha_{i}$ with a UE-cardinality of $N_{U, \alpha_{i}}$. Each UE $\mu_{j}$ is assumed to have a scheduling priority corresponding to each AP $\alpha_{i}$, which is given by the weight in (17). Let $\mathcal{P}_{\alpha_{i}}=\left(\omega_{d}\left(e_{\alpha_{i}, \mu_{j}}: \mu_{j} \in \mathcal{V}_{U, \alpha_{i}}\right)\right.$ denote the priority of each element of $\mathcal{V}_{U, \alpha_{i}}$ representing the AP $\alpha_{i}$. Furthermore, if a UE does not receive any connection request from any AP during the slot considered, it is said to be an idle UE; otherwise, it is an active UE. Let us now introduce our algorithm by considering Fig. 5a, for example.

i) Initial selection. Each VLC AP $\alpha_{i}$ selects the specific UE $\mu_{j}^{\alpha_{i}}$ from $\mathcal{V}_{U, \alpha_{i}}$ associated with the highest distance-based priority, which satisfies

$$
\mu_{j}^{\alpha_{i}}=\arg \max _{\mu_{j} \in \mathcal{V}_{U, \alpha_{i}}}\left(\mathcal{P}_{\alpha_{i}}\right)
$$

If the UE $\mu_{j}^{\alpha_{i}}$ receives an assignment request exclusively from the AP $\alpha_{i}$, this AP-UE pair is referred to
Fig. 5. (a) The network component considered. (b) Initial selection and tentative-cluster construction. The shaded triangles indicate the hitherto unsupported UEs. (c) Expansion of the tentative-cluster. (d) UC-VT cluster formation, where the incomplete ellipsoids indicate the specific UC-VT cooperation requests of the UEs and the finally unscheduled UEs are denoted by the triangles with dashed boundary.

as a Single-to-Single Matching (SSM), which may be 702 formally defined as

$$
\mathcal{M}_{\mathrm{SSM}}=\left\{\alpha_{i} \rightarrow \mu_{j}^{\alpha_{i}}: \forall \alpha_{i^{\prime}} \neq \alpha_{i} \Rightarrow \mu_{j}^{\alpha_{i^{\prime}}} \neq \mu_{j}^{\alpha_{i}}\right\}
$$

For example, as shown in Fig. 5b, $\mu_{4}$ only receives an 704 assignment request from $\alpha_{7}$, although it also falls within 705 the coverage of $\alpha_{11}$, since $\mu_{2}$ has the largest schedul- 706 ing weight of 4 for $\alpha_{11}$ and therefore the $\left\{\alpha_{11} \rightarrow \mu_{4}\right\} \quad 707$ link of weight 3 is ignored. Similarly the $\left\{\alpha_{6} \rightarrow \mu_{6}\right\}$ link 708 of weight 4 is also a SSM, because the $\left\{\alpha_{6} \rightarrow \mu_{5}\right\}$ and 709 $\left\{\alpha_{6} \rightarrow \mu_{7}\right\}$ links have a lower weight of 2 . Hence, the 710 AP-UE association after this initial selection is shown in 711 Fig. 5b, where the low-weight links are only shown with 712 dotted lines.

ii) Tentative-cluster construction. If a UE is offered multiple 714 connection opportunities by different APs, this is said to 715 be a Multiple-to-Single Matching (MSM), which may be 716 defined as

$$
\begin{array}{r}
\mathcal{M}_{\mathrm{MSM}}=\left\{\left(\alpha_{i}, \alpha_{i^{\prime}}, \alpha_{i^{\prime \prime}}, \ldots\right) \rightarrow \mu_{j}^{\alpha_{i}}:\right. \\
\left.\mu_{j}^{\alpha_{i}}=\mu_{j}^{\alpha_{i^{\prime}}}=\mu_{j}^{\alpha_{i^{\prime \prime}}}=\ldots\right\},
\end{array}
$$

where we have $\mathcal{M}_{\mathrm{MSM}}=\left\{\left(\alpha_{10}, \alpha_{11}\right) \rightarrow \mu_{2}\right\}$ in the exam- 718 ple of Fig. 5b, since $\mu_{2}$ has the highest priority for both 719 $\alpha_{10}$ and $\alpha_{11}$. Furthermore, each MSM is assumed to con- 720 struct a tentative-cluster, as also shown in Fig. 5b, where 721 the shaded triangles indicate the hitherto unsupported 722 UEs.

iii) Expansion of the tentative-cluster. Within a tentative- 724 cluster $\left(\alpha_{i}, \alpha_{i^{\prime}}, \alpha_{i^{\prime \prime}}, \ldots\right) \rightarrow \mu_{j}^{\alpha_{i}}$, each $\mathrm{AP} \alpha_{i}$ reselects a 725 
hitherto unsupported UE to be supported with the highest priority, provided that there are still unsupported UEs in $\mathcal{V}_{U, \alpha_{i}}$. Accordingly, as indicated by Fig. $5 \mathrm{c}, \alpha_{10}$ reselects the unsupported UE $\mu_{3}$, since the set $\mathcal{V}_{U}^{\alpha_{10}} \backslash\left(\mu_{2}, \mu_{6}\right)=$ $\mu_{3}$ is non-empty and $\mu_{3}$ is the only unsupported UE within the coverage of $\alpha_{10}$. However, since the set $\mathcal{V}_{U}^{\alpha_{11}} \backslash$ $\left(\mu_{2}, \mu_{4}\right)=\emptyset$ is empty, $\alpha_{11}$ does not have any additional UE to support.

iv) Cluster formation. In order to mitigate the inter-cluster interference, the scheduled UEs found in the overlapping areas of some neighbouring APs determine the cooperation of these APs. More explicitly, if a particular scheduled UE has the benefit of a LOS ray from several different APs, then the UE sends a cooperation request to these APs. For example, in Fig. 5d $\mu_{2}$ sends its cooperation request to $\left\{\alpha_{10}, \alpha_{11}\right\}$, while $\mu_{4}$ and $\mu_{6}$ request cooperation with $\left\{\alpha_{11}, \alpha_{7}\right\}$ and $\left\{\alpha_{10}, \alpha_{6}\right\}$, respectively, as indicated by the incomplete ellipsoids. Thus all the cooperating APs and their matching UEs construct a single UC-VT cluster in the examples of Fig. $1 \mathrm{~b}$.

Recall that $N_{A}$ APs are only capable of simultaneously supporting at most the same number of UEs according to (14). Therefore, during the expansion of the tentative-cluster, the number of active UEs becomes $\left(N_{A}+1\right)$, provided that all APs can connect with an idle UE. Hence, the UE having the smallest priority is removed. Let us now provide an overview of the greedy cluster formation/MUS technique in form of Algorithm 1.

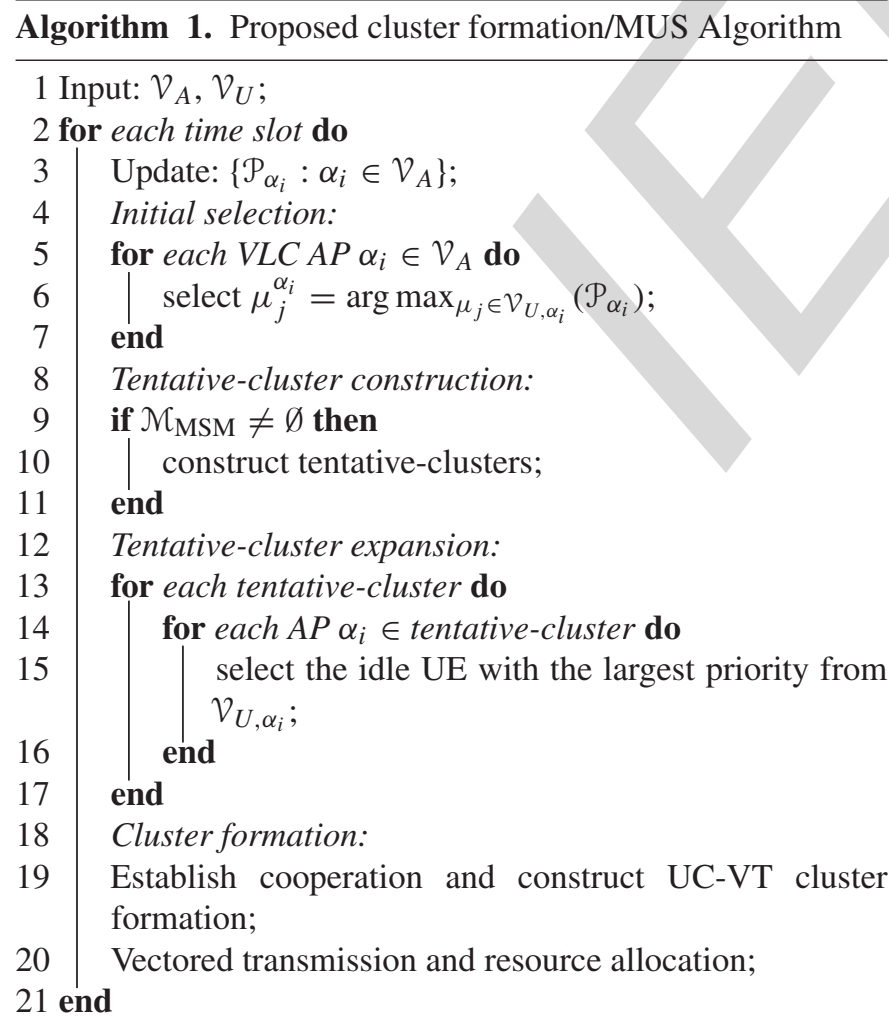

\section{Performance Evaluation}

In this section, we will present our simulation results characterising the MUS and cluster formation algorithms,
TABLE II

SIMULATION PARAMETERS

\begin{tabular}{l|r}
\hline \hline Transmitted optical power per LED lamp $\left(P_{t}\right):$ & $20[\mathrm{~W}]$ \\
$20 \times 20$ LEDs with 50mW per LED & $55^{\circ} / 57.5^{\circ} / 60^{\circ} / 62.5^{\circ}$ \\
Half of the receiver's FOV $\left(\psi_{\mathrm{F}}\right)$ & 0.8 \\
Reflectance factor $(\rho)$ & $14.4[\mathrm{~W}]$ \\
$\begin{array}{l}\text { Power of circled-LED lamp: } 17 \times 17 \text { LEDs } \\
\text { with 50mW per LED }\end{array}$ & $36.8[\mathrm{~W}]$ \\
$\begin{array}{l}\text { Power of cornered-LED lamp: } 23 \times 32 \text { LEDs } \\
\text { with 50mW per LED }\end{array}$ & \\
\hline \hline
\end{tabular}

with a special emphasis on our UC-VT cluster formation. A 781 $15 \mathrm{~m} \times 15 \mathrm{~m} \times 3 \mathrm{~m}$ room model is considered, which is covered 782 by a VLC down-link including $(4 \times 4)$ uniformly distributed 783 optical APs at a height of $2.5 \mathrm{~m}$. The parameters of the 784 LED arrays are summarized in TABLE II. Our investigations 785 include both the LOS and the first reflected light-path, where 786 the channel's DC attenuation is given by (1) and (2), respec- 787 tively. Furthermore, as mentioned in Section II, ACO-OFDM 788 is considered and the associated capacity is given as $R=789$ $\frac{B}{4} \log _{2}(1+\xi)$ according to [9], where $\xi$ is the SINR of (8). Our 790 simulation results were averaged over 50 independent snap- 791 shots and each snapshot is constituted by 50 consecutive time 792 slots having a length of $1 \mathrm{~ms}$. The UEs at a height of $2.5 \mathrm{~m}$ are 793 random uniformly distributed at the beginning of each snapshot 794 and they move randomly during the consecutive 50 time slots 795 at a speed of $1 \mathrm{~m} / \mathrm{s}$. The locations of the UEs are reported every 796 time slot, i.e. every $1 \mathrm{~ms}$.

\section{A. Complexity Analysis}

As shown in Fig. 6a, when the number of UEs is less than 799 5 , the exhaustive search may be an appealing low-complexity 800 approach of finding the optimal solution for our joint opti- 801 mization problem. However, the number of possible cluster 802 formations found by employing the exhaustive search may 803 become excessive with the number of UEs increased. Even 804 if there are only 16 UEs supported by $16 \mathrm{APs}$, the average 805 number of possible formations becomes as high as $5 \times 10^{6} 806$ in a single simulation run. By contrast, the complexity of the 807 KM-algorithm based approach may become inadequate in low- 808 UE-density scenarios. However, when the number of UEs is 809 higher than that of the APs, the complexity is only linearly 810 increased with the number of UEs, according to [26]. Fig. 6b 811 shows both the normalized throughput and the sum utility of 812 various cluster/cell formations, where the traditional NC cell 813 formation designs relying on UFR and on the FR factor of 814 two (FR-2) are considered as our benchmarkers. We adopt the 815 MUS algorithm for the UFR and FR-2 discussed in our previous 816 work [15]. Both the highest throughput attained and the sum 817 utility are quantified for the proposed UC-VT cluster forma- 818 tion, whose optimal solution is found by the exhaustive search. 819 The optimal MWM provides a similar solution as our proposed 820 greedy algorithm, both of which are about $90 \%$ of the opti- 821 mal exhaustive search-based value in the scenario considered. 822 Therefore, we will omit the optimal exhaustive search in the 823 

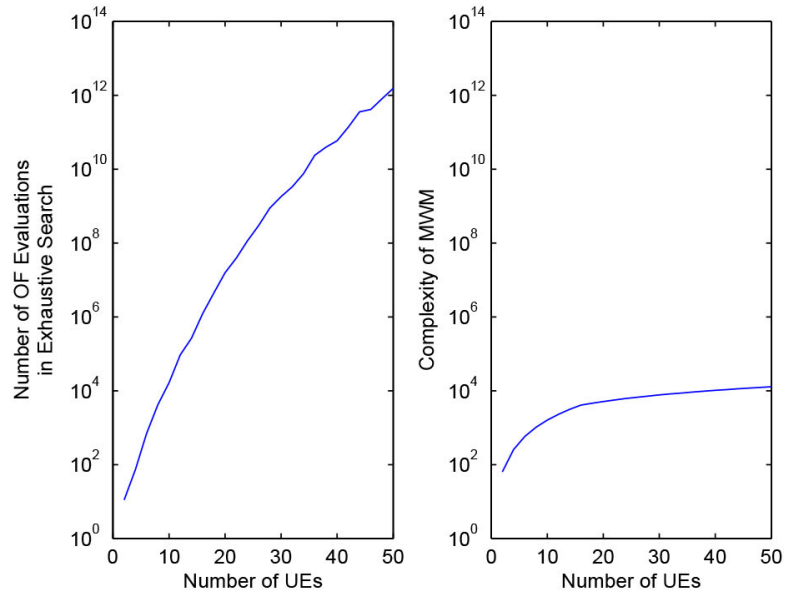

(a)

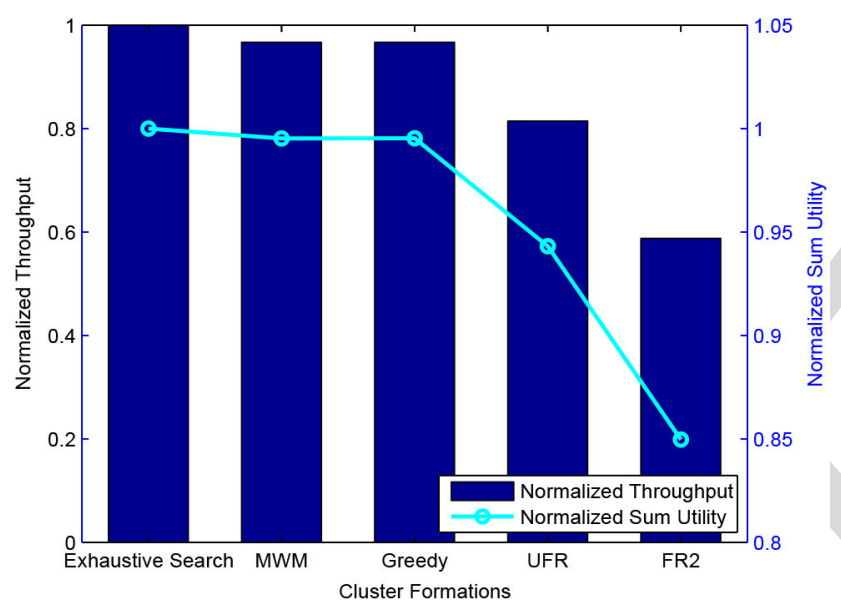

(b)

Fig. 6. (a) The complexity of the exhaustive search for finding the optimal UCVT cluster formations and the complexity of KM-algorithm based MWM for finding a suboptimal cluster formation solution; (b) The normalized throughput and the normalized sum utility/OF value, where $\mathrm{FOV}=110^{\circ}$ and $10 \mathrm{UEs}$ are assumed moving randomly at a speed of $1 \mathrm{~m} / \mathrm{s}$.

rest of this treatise and we opt for the MWM solution as well as for the more practical greedy algorithm for finding the UC-VT cluster formation solution.

\section{B. Throughput Investigations}

1) Throughput Investigations for Various FOV and UE Density: Since the FOV is an influential parameter in VLC networks in Fig. 7a, we consider its effect on the system's performance. The average throughput per UE is reduced, when the $\mathrm{FOV}^{3}$ is increased, due to the increased interference, while our proposed UC-VT cluster formation remains superior in all scenarios considered. In particular, observe in Fig. 7a that the UFR

\footnotetext{
${ }^{3}$ In order to evaluate the system's performance for various FOVs, we selected $110^{\circ} / 115^{\circ}$ and $120^{\circ} / 125^{\circ}$. In the former scenario, the UE is capable of receiving data from two neighboring APs and the area contaminated by potential interference is modest. When the FOV is increased to $120^{\circ} / 125^{\circ}$, the UE is capable of receiving data from four APs and the potential interferencecontaminated area is also increased. These four FOVs correspond to different interference levels, although their absolute values are quite similar.
}

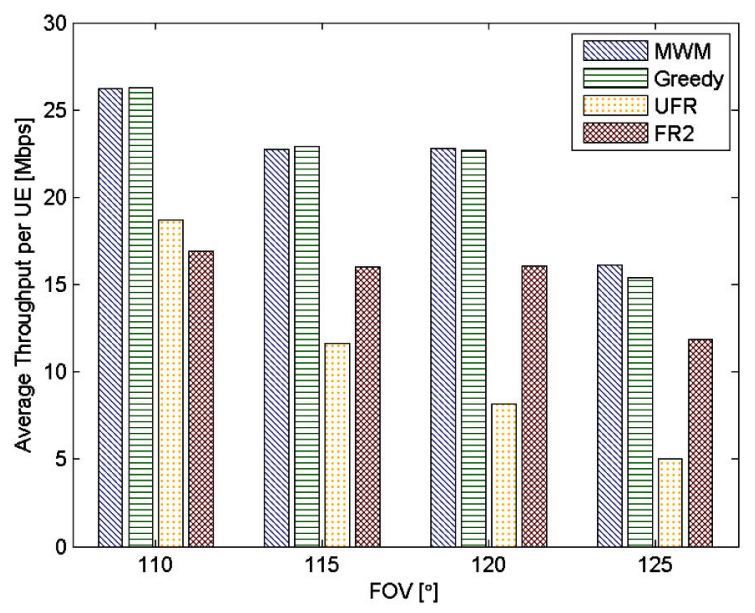

(a)

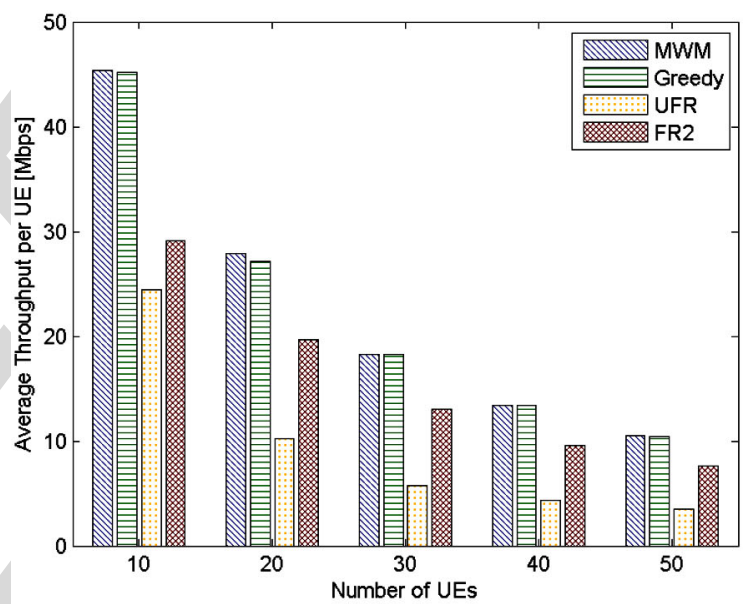

(b)

Fig. 7. (a) Average throughput per UE provided by different cluster formation/cell formation schemes for various FOVs and for 25 UEs. (b) Average throughput per UE provided by different cluster formation/cell formation schemes for various UE densities, where the FOV is $120^{\circ}$ and the number of UEs is 25 .

design exhibits the worst interference immunity and offers the 835 lowest throughput, when the FOV is higher than $115^{\circ}$. Fig. $7 \mathrm{~b} 836$ shows the average throughput per UE provided by different 837 cluster formation/cell formation schemes associated with var- 838 ious UE densities, where the FOV is $120^{\circ}$. As expected, our 839 proposed UC-VT cluster formation is capable of providing the 840 highest average throughput for all the UE densities considered. 841

2) Throughput Investigations for Various LOS Blocking 842 Probabilities: As mentioned in Section I, the performance of 843 VLC systems is expected to be seriously degraded in non-LOS 844 scenarios. In order to investigate the non-LOS behaviour of this 845 VLC system, we introduce the LOS blocking probability $P_{b} 846$ and assume that the achievable data rate $\tilde{R}$ obeys a Bernoulli 847 distribution [13], with the probability mass function of:

$$
f(\tilde{R})=\left\{\begin{array}{lll}
1-P_{b}, & \text { if } & \tilde{R}=R_{s}, \\
P_{b}, & \text { if } & \tilde{R}=R_{r}
\end{array}\right.
$$

where $R_{s}$ and $R_{r}$ denote the achievable data rate of the UE 849 either in the presence or absence of LOS reception. Then the 850 


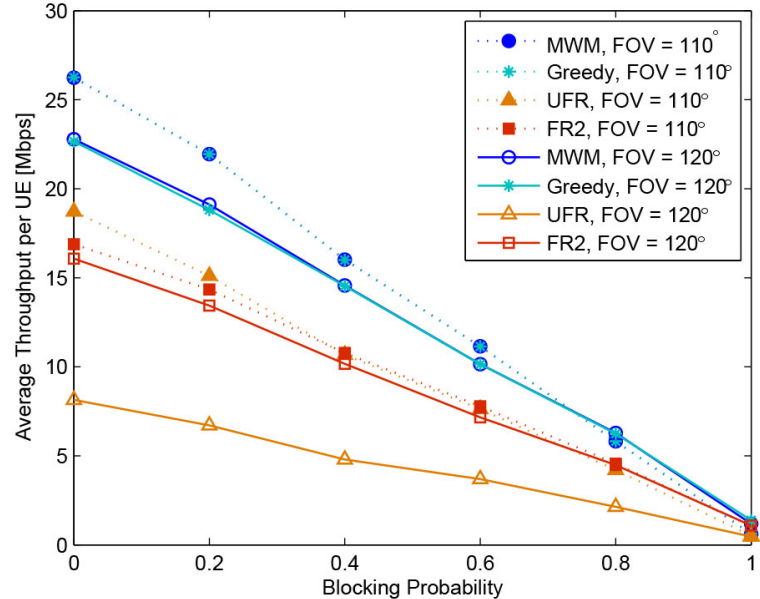

Fig. 8. Average UE throughput of our VLC system for various blocking probabilities and FOVs supporting 25 UEs in each scenario.

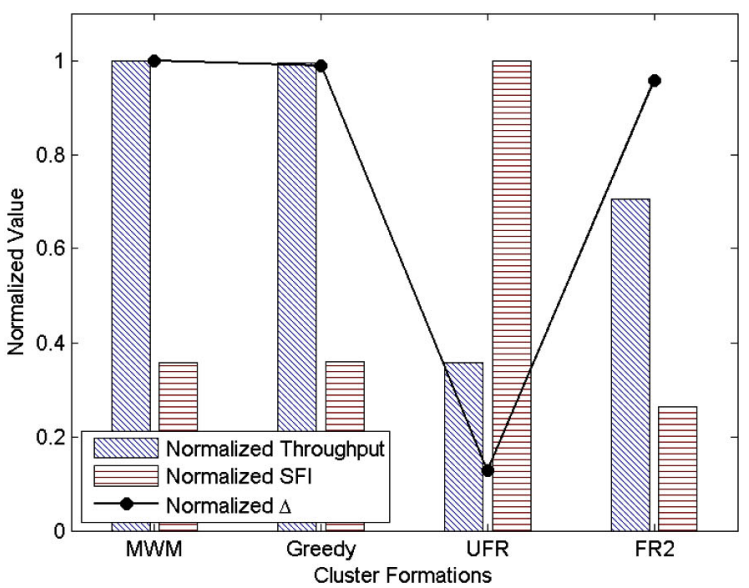

(a)

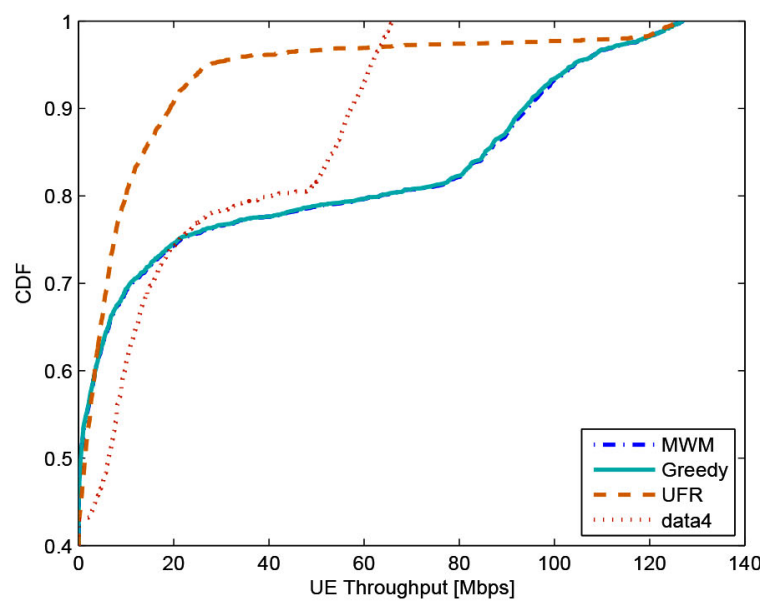

(b)

Fig. 9. (a) The normalized average throughput and the Service Fairness Index (SFI) of various cluster formation/cell formation schemes; (b) CDF of the UE throughput, where the number of UEs is 25 and we have $\mathrm{FOV}=120^{\circ}$.

VLC down-link data rate may be written as $\tilde{R}=P_{b} \cdot R_{r}+$ $\left(1-P_{b}\right) \cdot R_{s}$. At this stage, we assume that all LOS paths are blocked with an equal probability. As shown in Fig. 8, the

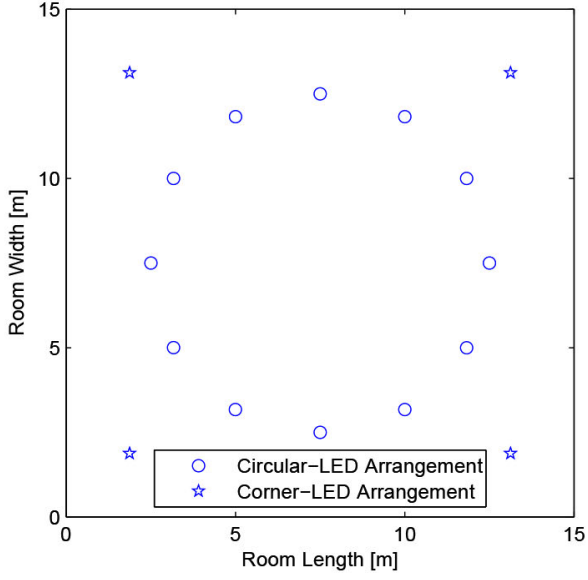

(a)

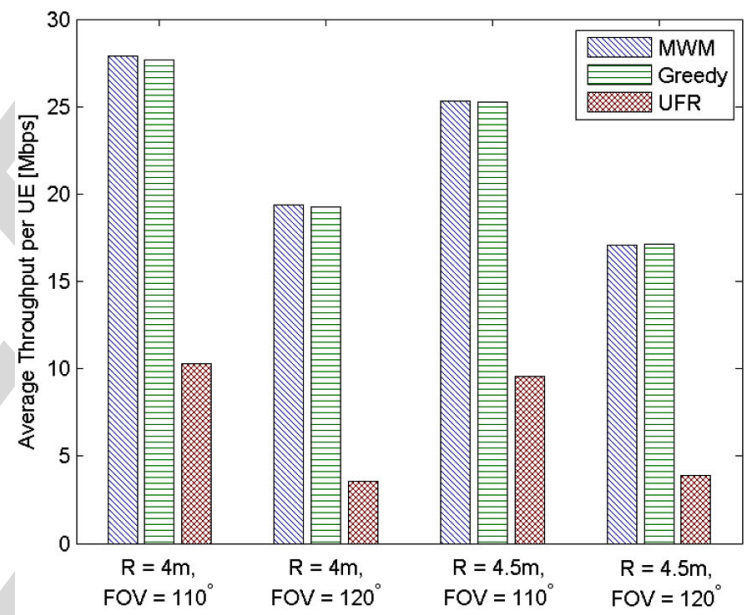

(b)

Fig. 10. (a) shows the LED-arrangement, where the LED circle has a radius of $4.5 \mathrm{~m}$ and the corner LEDs are at $1.875 \mathrm{~m}$ from the walls. (b) System performance of the LED arrangement seen in (a) for 25 UEs.

average UE throughput attained is reduced upon increasing the 854 LOS blocking probability in all the scenarios considered, but 855 our UC-VT cluster formation still achieves a higher throughput. 856 Furthermore, the system performance of the MWM approach 857 and of our proposed greedy cluster formation/MUS algorithm 858 remains quite similar, regardless of the specific blocking prob- 859 ability and FOV.

\section{Fairness Investigations}

In order to investigate the grade of fairness experienced by 862 the UEs, the Service Fairness Index (SFI) of [31] is introduced. 863 The objective of ensuring fairness amongst the UEs is to guar- 864 antee that all UEs benefit from the same throughput within a 865 given period, provided that the UEs' data rate requirements are 866 identical [12], which is often unrealistic. The SFI was defined 867 as [31]: 


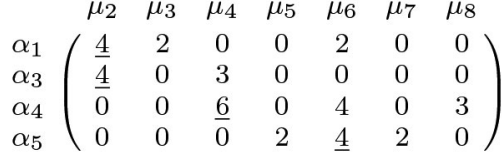

(a)

$$
\left(\begin{array}{ccccccc}
0^{*} & 2 & 4 & 4 & 2 & 4 & 4 \\
0 & 4 & 1 & 4 & 4 & 4 & 4 \\
6 & 6 & 0^{*} & 6 & 2 & 6 & 3 \\
4 & 4 & 4 & 2 & 0^{*} & 2 & 4
\end{array}\right)
$$

(b)

$\left(\begin{array}{ccccccc}0^{*} & 0^{\prime} & 4 & 2 & 2 & 2 & 2 \\ 0^{\prime} & 2 & 1 & 2 & 4 & 2 & 2 \\ 6 & 4 & 0^{*} & 4 & 2 & 4 & 1 \\ 4 & 2 & 4 & 0 & 0^{*} & 0 & 2\end{array}\right)$

(d)

$\left(\begin{array}{ccccccc}0^{*} & 0^{\prime} & 4 & 2 & 2 & 2 & 2 \\ 0 & 2 & 1 & 2 & 4 & 2 & 2 \\ \phi & 4 & 0^{*} & 4 & 2 & 4 & 1 \\ 4 & 2 & 4 & 0 & 0^{*} & 0 & 2\end{array}\right)$

(c)

(e)

Fig. 11. (a) The weight matrix $\left(\omega_{d}\left(e_{\alpha_{i}, \mu_{j}}\right)\right)$ of $Q_{2}$, where the maximum element of each row is underlined. (b) Initialization step. The equivalent matrix $\left(a_{\alpha_{i}, \mu_{j}}\right)$ of $\left(\omega_{d}\left(e_{\alpha_{i}, \mu_{j}}\right)\right)$ is obtained as $\left(a_{\alpha_{i}, \mu_{j}}\right)=\left(c_{\alpha_{i}}-\omega_{d}\left(e_{\alpha_{i}, \mu_{j}}\right)\right)$, where we have $\left(c_{\alpha_{i}}\right)=[4,4,6,4]^{\mathrm{T}}$. Find and mark the zero by a star, if there are no starred zeros in its row or in its column. Cover every column containing a $0^{*}$ by a vertical line. (c) Adjustment step. $\left(a_{\alpha_{i}, \mu_{j}}\right)$ is modified as $\left(a_{\alpha_{i}, \mu_{j}}-c_{\alpha_{i}}+\right.$ $\left.r_{\mu_{j}}\right)$, where $\left(c_{\alpha_{i}}\right)=[0,0,0,0]^{\mathrm{T}}$ and $\left(r_{\mu_{j}}\right)=[0,-2,0,-2,0,-2,-2]$. Mark the uncovered zeros by the upper prime. (d) Explicitly, if there is a starred zero in the primed zero's row, mark this row by a line and remove the vertical line for the column of the starred zero. (e) Starred zero and primed zero alternating. Remove all lines. Recover the columns containing $0^{*}$. Optimal solution found.

which reflects the maximum throughput-difference of different UEs. If the SFI is low, the throughput-difference is low and the UEs are served fairly, while if the SFI is high, the UEs experiencing a lower data rate may complain about their unfair treatment. Furthermore, by jointly considering the throughput, we may define

$$
\Delta=\frac{\text { Average throughput per UE }}{\text { SFI }} .
$$

Hence, $\Delta$ constitutes a comprehensive system performance metric, joint characterising both the throughput as well as the service fairness. If $\Delta$ is low, the system either provides a low throughput or a poor fairness; and vice versa. Fig. 9a shows the normalized throughput and SFI of various cell formations and cluster formations, where the UFR design has the worst performance associated with the lowest $\Delta$. Moreover, the Cumulative Distribution Function (CDF) of the UE throughput is shown in Fig. 9b. It can be seen that the UE may have as high as $40 \%$ probability of remaining unserved during each time slot in all the scenarios considered.

\section{Irregular VLC AP Arrangements}

Our proposed UC-VT cluster formation and MUS scheme may be readily applied to arbitrary topologies. Let us consider Fig. 10a, for example. This specific VLC AP arrangement was advocated in [32] for reducing the Signal-to-Noise-Ratio (SNR) fluctuation and was also employed in [16] for implementing a scheduling algorithm. As shown in Fig. 10a, 12 LED lamps constitute a circle and 4 LED lamps are placed in the corners at a height of $2.5 \mathrm{~m}$, which are referred to here as the circular- 894 LED arrangement and corner-LED arrangement, respectively. 895 The power of each LED array is $14.4 \mathrm{~W}$ and $36.8 \mathrm{~W}$ in the 896 circular- and corner-arrangements of our simulations. Thus the 897 total number of optical APs remains 16 and the sum of their 898 transmission power is at most $320 \mathrm{~W}$, which is the same as 899 that of the regular $(4 \times 4)$ LED array arrangement. Fig. 10b 900 shows the average throughput per UE for the LED arrangement 901 of Fig. 10b. The average throughput is slightly reduced, when 902 the radius of the LED circle is increased from $4 \mathrm{~m}$ to $4.5 \mathrm{~m}, 903$ but our proposed UC-VT cluster formation still outperforms the 904 traditional cell formation design in all scenarios of this circular 905 LED arrangement.

\section{CONCLUSiOnS}

In this paper, an amorphous UC-VT cluster formation was 908 proposed for mitigating the ICI and to allow a single cluster 909 to support multiple UEs. The MUS problem combined with 910 our UC-VT cluster formation was investigated and the opti- 911 mal solution was found by an exhaustive search approach. 912 Since the exhaustive search may become complex, the origi- 913 nal joint problem was reformulated as a MWM problem, which 914 was solved by the classic KM-algorithm-based method. In 915 order to further reduce the computational complexity, an effi- 916 cient greedy MUS algorithm was proposed for constructing our 917 UC-VT clusters. Our simulation results demonstrated that the 918 UC-VT cluster formation is capable of providing a higher aver- 919 age UE throughput than the traditional NC cell designs in all 920 the scenarios considered. Despite the promise of the UC-VT 921 cluster formation, naturally, some challenges arise when incor- 922 porating our system-level UC design into VLC environments. 923 The open challenges may be highlighted from various perspec- 924 tives, including the acquisition of accurate location information, 925 the research of robustness to LOS blocking, the technology 926 counterpart to be used for up-link support, etc.

\section{APPENDIX A}

\section{KM-ALGORITHM-BASED APPROACH FOR FINDING} THE OPTIMAL MWM

Let us first rely on Lemma 1, where having independent ele- 931 ments indicates that none of them occupies the same row or 932 column.

Lemma 1: (König Theorem)[26]. If $z$ is the maximum num- 934 ber of independent zero elements in the matrix $\left(a_{\alpha_{i}, \mu_{j}}\right)$, then 935 there are $z$ lines (rows, columns or both) containing all the zeros 936 elements of $\left(a_{\alpha_{i}, \mu_{j}}\right)$.

First, the weight matrix $\left(\omega_{d}\left(e_{\alpha_{i}, \mu_{j}}\right)\right)$ of Fig. 3a is formu- 938 lated, as shown in Fig. 11a, where the weight is set to zero 939 when there is no link between two vertices. Our problem is 940 that of maximizing the sum weight, while the KM algorithm 941 is suitable for a minimization problem. We have to construct 942 an equivalent matrix $\left(a_{\alpha_{i}, \mu_{j}}\right)$ for $\left(\omega_{d}\left(e_{\alpha_{i}, \mu_{j}}\right)\right)$, according to 943 Theorem 1. The maximum element $\left(\omega_{d}\left(e_{\alpha_{i}, \mu_{j}}\right)\right)$ is selected and 944 forms $\left(c_{\alpha_{i}}\right)$, where we have $\left(c_{\alpha_{i}}\right)=[4,4,6,4]^{\mathrm{T}}$ in our exam- 945 ple. Let $\left(c_{\alpha_{i}}-\omega_{d}\left(e_{\alpha_{i}, \mu_{j}}\right)\right)$ be the matrix $\left(a_{\alpha_{i}, \mu_{j}}\right)$, as shown in 946 Fig. 11b, and its optimal matching solution minimizing the sum 947 
weight is also optimal for our MWM problem. Next, find a zero in each column of $\left(c_{\alpha_{i}}-\omega_{d}\left(e_{\alpha_{i}, \mu_{j}}\right)\right)$. If however there is no starred zero either in its row or in its column, we mark it by a star, again as shown in Fig. 11b. Then we mark every column containing a $0^{*}$ by a vertical line and all the $0^{*}$ form a set of independent zeros, since none of them occupies the same row or column. The above-mentioned procedure is our initialization step, which may be described as:

i) Initialization. Generate an initial label set $\left(c_{\alpha_{i}}\right)$, where for each row $\alpha_{i}$ we have:

$$
c_{\alpha_{i}}=\max _{\mu_{j}}\left(\omega_{d}\left(e_{\alpha_{i}, \mu_{j}}\right)\right), \quad \mu_{j}=1, \ldots, N_{U, Q_{m}} .
$$

Thus, the equivalent matrix is constructed as $\left(c_{\alpha_{i}}-\right.$ $\left.\omega_{d}\left(e_{\alpha_{i}, \mu_{j}}\right)\right)$. Generate an initial matching $\mathcal{M}_{Q_{m}}$ by finding and marking independent zeros denoted by $z_{j}^{\left(\alpha_{i}, \mu_{j}\right)}$ using a star, whose superscript corresponds to its index in $\left(c_{\alpha_{i}}-\omega_{d}\left(e_{\alpha_{i}, \mu_{j}}\right)\right)$, where we have:

$$
\begin{aligned}
& \forall z_{j}^{\left(\alpha_{i}, \mu_{j}\right)} \in\left(z_{j}\right), \quad z_{j^{\prime} \neq j}^{\left(\alpha_{i}^{\prime} \neq \alpha_{i}, \mu_{j}\right)} \notin\left(z_{j}\right), \\
& \forall z_{j}^{\left(\alpha_{i}, \mu_{j}\right)} \in\left(z_{j}\right), \quad z_{j^{\prime} \neq j}^{\left(\alpha_{i}, p_{\alpha_{i}^{\prime}} \neq \mu_{j}\right)} \notin\left(z_{j}\right) .
\end{aligned}
$$

If $\left|\left(z_{j}\right)\right|=\min \left\{N_{A, Q_{m}}, N_{U, Q_{m}}\right\}$ columns are marked, we find the desired matching, where each AP matches a specific UE and the sum weight of their links is maximized, which furthermore form a UC-VT cluster. Otherwise, the cardinality of the matching will be iteratively increased during the following steps.

If there are no unmarked zeros as shown in Fig. 11b, the current matrix should be modified according to Theorem 1, which leads to the following adjustment step.

ii) Adjustment. Let $h$ be the smallest unmarked element of the matrix and construct a column vector $\left(c_{\alpha_{i}}\right)$ and a row vector $\left(r_{\mu_{j}}\right)$ by the following rules: if the $\alpha_{i}$ th row is covered, $c_{\alpha_{i}}=h$; otherwise, $c_{\alpha_{i}}=0$. If the $\mu_{j}$ th column is covered, $r_{\mu_{j}}=0$; otherwise, $r_{\mu_{j}}=-h$. In our example, $\left(a_{\alpha_{i}, \mu_{j}}\right)$ is updated as $\left(a_{\alpha_{i}, \mu_{j}}-c_{\alpha_{i}}+r_{\mu_{j}}\right)$ and $\left(c_{\alpha_{i}}\right)=$ $[0,0,0,0]^{\mathrm{T}}$ and $\left(r_{p_{i}}\right)=[0,-2,0,-2,0,-2,-2]$, as shown in Fig. 11c.

Then let us choose and mark an unmarked zero by priming it. If there is a starred zero in its row, mark this row by a line and remove the line from the column of the starred zero, as shown in Fig. 11d. Then we prime another unmarked zero in the second row indicated by the bold font, but there is no starred zero in its row. According to the starred and primed zero alternating rules of [26], we obtain the matrix seen in Fig. 11e, where the number of independent zeros reached its maximum given by the number of rows. Correspondingly, the number of lines containing all these zeros becomes maximal, as stated by Lemma 1, where the maximum number of independent zeros is equal to the number of lines containing them. The algorithm terminates here in our scenario. However, if the number of marked columns is still insufficient, the set of independent zeros has to be increased by iteratively repeating the above-mentioned steps, commencing from the Adjustment stage. Thus, we find the optimal solution for our MWM, which is $\left\{\alpha_{10} \rightarrow \mu_{3}, \alpha_{11} \rightarrow \mu_{2}, \alpha_{7} \rightarrow\right.$ $\left.\mu_{4}, \alpha_{6} \rightarrow \mu_{6}\right\}$, namely the same as indicated in Fig. $3 \mathrm{~b}$.
REFERENCES

[1] L. Hanzo, H. Haas, S. Imre, D. O’Brien, M. Rupp, and L. Gyongyosi, 999 "Wireless myths, realities, and futures: From 3G/4G to optical and 1000 quantum wireless," in Proceedings of the IEEE, vol. 100, May 2012, 1001 pp. $1853-1888$.

1002

[2] D. O'Brien, H. Haas, S. Rajbhandari, H. Chun, G. Faulkner, K. Cameron, 1003 A. V. Jalajakumari, R. Henderson, D. Tsonev, M. Ijaz et al., "Integrated 1004 multiple-input multiple-output visible light communications systems: 1005 recent progress and results," in SPIE OPTO, 2015, pp. 93 870P-93 870P. 1006

[3] D. Tsonev, S. Videv, and H. Haas, "Towards a $100 \mathrm{~Gb} / \mathrm{s}$ visible light 1007 wireless access network," Optics Express, vol. 23, no. 2, pp. 1627-1637, 1008 Jan 2015.

1009

[4] D. Tsonev, H. Chun, S. Rajbhandari, J. McKendry, S. Videv, E. Gu, 1010 M. Haji, S. Watson, A. Kelly, G. Faulkner, M. Dawson, H. Haas, and 1011 D. O'Brien, "A 3-Gb/s single-LED OFDM-based wireless VLC link 1012 using a gallium nitride $\mu$ LED," IEEE Photonics Technology Letters, 1013 vol. 26, no. 7, pp. 637-640, April 2014.

[5] S. Dissanayake and J. Armstrong, "Comparison of ACO-OFDM, DCO- 1015 OFDM and ADO-OFDM in IM/DD systems," Journal of Lightwave 1016 Technology, vol. 31, no. 7, pp. 1063-1072, Apr. 2013.

1017

[6] A. Azhar, T. Tran, and D. O'Brien, "A Gigabit/s indoor wireless 1018 transmission using MIMO-OFDM visible-light communications," IEEE 1019 Photonics Technology Letters, vol. 25, no. 2, pp. 171-174, Jan 2013.

[7] J. Grubor, S. Randel, K.-D. Langer, and J. Walewski, "Broadband 1021 information broadcasting using LED-based interior lighting," Journal of 1022 Lightwave Technology, vol. 26, no. 24, pp. 3883-3892, Dec. 2008.

[8] J. Armstrong and B. Schmidt, "Comparison of asymmetrically 1024 clipped optical OFDM and DC-biased optical OFDM in AWGN," 1025 Communications Letters, IEEE, vol. 12, no. 5, pp. 343-345, May 1026 2008.

1027

[9] X. Li, R. Mardling, and J. Armstrong, "Channel capacity of IM/DD opti- 1028 cal communication systems and of ACO-OFDM," in IEEE ICC 2007, 1029 June 2007, pp. 2128-2133.

[10] T. Komine and M. Nakagawa, "Fundamental analysis for visible- 1031 light communication system using LED lights," IEEE Transactions on 1032 Consumer Electronics, vol. 50, no. 1, pp. 100-107, Feb. 2004.

[11] "IEEE standard for local and metropolitan area networks-part 15.7: 1034 Short-range wireless optical communication using visible light," IEEE 1035 Std 802.15.7-2011, pp. 1-309, Sep. 2011.

[12] X. Li, R. Zhang, and L. Hanzo, "Cooperative load balancing in 1037 hybrid visible light communications and WiFi," IEEE Transactions on 1038 Communications, vol. PP, no. 99, pp. 1-1, Mar. 2015. 1039

[13] F. Jin, R. Zhang, and L. Hanzo, "Resource allocation under delay- 1040 guarantee constraints for heterogeneous visible-light and RF femtocell," 1041 IEEE Transactions on Wireless Communications, vol. 14, no. 2, pp. 1020- 1042 1034, Feb 2015.

[14] C. Chen, N. Serafimovski, and H. Haas, "Fractional frequency reuse in 1044 optical wireless cellular networks," in IEEE PIMRC 2013, Sep. 2013, 1045 pp. 3594-3598.

[15] X. Li, R. Zhang, J. Wang, and L. Hanzo, "Cell-Centric and User-Centric 1047 Multi-User scheduling in visible light communication aided networks," 1048 in IEEE ICC 2015 (06) ONS, Jun. 2015.

1049

[16] Y. Tao, X. Liang, J. Wang, and C. Zhao, "Scheduling for indoor visi- 1050 ble light communication based on graph theory," Optics Express, vol. 23, 1051 no. 3, pp. 2737-2752, Feb 2015.

[17] R. Zhang, J. Wang, Z. Wang, Z. Xu, C. Zhao, and L. Hanzo, "Visible light 1053 communications in heterogeneous networks: Paving the way for user- 1054 centric design," IEEE Wireless Communications, vol. 22, no. 2, pp. 8-16, 1055 April 2015 .

[18] D. Bykhovsky and S. Arnon, "Multiple access resource allocation in vis- 1057 ible light communication systems," Journal of Lightwave Technology, 1058 vol. 32, no. 8, pp. 1594-1600, April 2014.

[19] M. Biagi, S. Pergoloni, and A. Vegni, "Last: a framework to local- 1060 ize, access, schedule and transmit in indoor VLC systems," Journal of 1061 Lightwave Technology, vol. PP, no. 99, pp. 1-1, 2015.

[20] X. Huang, X. Fu, and W. Xu, "Incremental scheduling scheme for indoor 1063 visible light communication," Electronics Letters, vol. 51, no. 3, pp. 268- 1064 270, Feb 2015.

1065

[21] O. Babatundi, L. Qian, and J. Cheng, "Downlink scheduling in visible 1066 light communications," in WCSP 2014, Oct 2014, pp. 1-6. 1067

[22] H. Kushner and P. Whiting, "Convergence of proportional-fair sharing 1068 algorithms under general conditions," IEEE Transactions on Wireless 1069 Communications, vol. 3, no. 4, pp. 1250-1259, July 2004.

[23] J. Akhtman and L. Hanzo, "Power versus bandwidth-efficiency in wire- 1071 less communications: The economic perspective," in IEEE VTC 2009, 1072 Sep. 2009, pp. 1-5. 


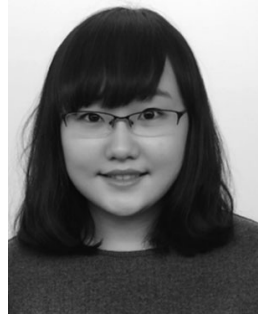

Xuan $\mathbf{L i}$ received the B.Eng. degree in optical information science and technology from Beijing Institute of Technology, Beijing, China, in 2012. She is currently pursuing the Ph.D. degree at the Southampton Wireless Group, University of Southampton, Southampton, U.K. Her research interests include visible light communications, heterogeneous networks, resource allocation, and scheduling algorithms.

111

1112

1113

1114

1115

1116

1117

1118

1119

1120

1121

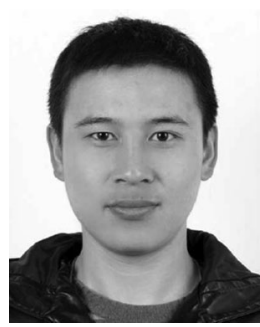

Fan Jin received the B.Sc. degree from Huazhong University of Science and Technology (HUST), Wuhan, China, and the Ph.D. degree from the University of Southampton, Southampton, U.K. in 2010 and 2015, respectively. He is currently working as an Engineer at Huawei, China. He was the recipient of a scholarship under the U.K.-China Scholarships for Excellence Programme. His research interests include multiuser communications, radio resource allocation, spectrum sensing and interference management in femtocells, and heterogeneous networks.

1122

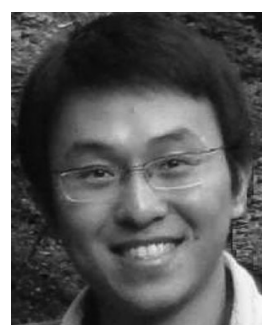

Rong Zhang (M'09) received the B.Sc. degree from the Southeast University, Nanjing, China, and the Ph.D. degree from Southampton University, Southampton, U.K. in 2003 and 2009, respectively. Before obtaining the doctorate, he was an Engineer (August 2003-July 2004) with China Telecom and a Research Assistant (January 2006-May 2009) with Mobile Virtual Center of Excellence (MVCE), U.K. After being a Postdoctoral Researcher (August 2009July 2012) with Southampton University, he took an industrial consulting leave (August 2012-January 2013) for Huawei Sweden R\&D as a System Algorithms Specialist. Since February 2013, he has been a Lecturer with the CSPC Group, ECS, Southampton University. He has authored more than 40 journals in prestigious publication avenues (e.g., IEEE and OSA) and many more in major conference proceedings. He regularly serves as a Reviewer for IEEE transactions/journals and has several times been a TPC member/invited session chair of major conferences. He was the recipient of joint funding from MVCE and EPSRC and is also a Visiting Researcher under the Worldwide University Network (WUN).

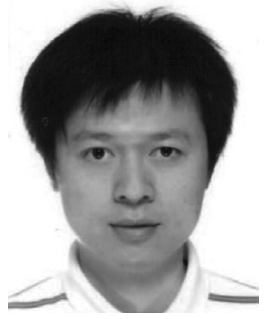

Jiaheng Wang (S'08-M'10-SM'14) received the 1141 B.E. and M.S. degrees from the Southeast University, 1142 Nanjing, China, in 2001 and 2006, respectively, and 1143 the Ph.D. degree in electrical engineering from the 1144 Hong Kong University of Science and Technology, 1145 Kowloon, Hong Kong, in 2010. He is currently 1146 an Associate Professor with the National Mobile 1147 Communications Research Laboratory (NCRL), 1148 Southeast University. From 2010 to 2011, he was 1149 with the Signal Processing Laboratory, ACCESS 1150 Linnaeus Center, KTH Royal Institute of Technology, 1151 Stockholm, Sweden. He also held a Visiting position with the Department 1152 of Computer and Information Science, University of Macau, Macau, China. 1153 His research interests include optimization in signal processing, wireless 1154 communications, and networks. He serves as an Associate Editor for the 1155 IEEE Signal Processing LetTERs. He was the recipient of the Humboldt 1156 Fellowship for Experienced Researchers and the Best Paper Award in WCSP 1157 2014.

1158

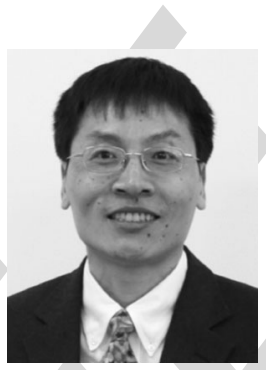

Zhengyuan Xu received the B.S. and M.S. degrees 1159 from Tsinghua University, Beijing, China, in 19891160 and 1991, respectively, and the Ph.D. degree 1161 from Stevens Institute of Technology, Hoboken, 1162 NJ, USA, in 1999. From 1991 to 1996, he was 1163 with Tsinghua Unisplendour Group Corporation, 1164 Tsinghua University, as System Engineer and 1165 Department Manager. In 1999, he joined the 1166 University of California, Riverside, CA, USA, first 1167 as Assistant Professor and then Tenured Associate 1168 Professor and Professor. He was the Founding 1169 Director of the Multicampus Center for Ubiquitous Communication by Light 1170 (UC-Light), University of California. In 2010, he was selected by the 1171 "Thousand Talents Program" of China, appointed as Professor with Tsinghua 1172 University, and then joined the University of Science and Technology of China 1173 (USTC). He is the Founding Director of the Optical Wireless Communication 1174 and Network Center, Founding Director of Wireless-Optical Communications 1175 Key Laboratory of Chinese Academy of Sciences, and Vice Dean of School of 1176 Information Science and Technology, USTC. He is also a Chief Scientist of the 1177 National Key Basic Research Program (973 Program) of China. His research 1178 interests include wireless communication and networking, optical wireless 1179 communications, geolocation, intelligent transportation, and signal processing. 1180 He has authored over 200 journal and conference papers. He has served as an 1181 Associate Editor and Guest Editor for different IEEE and OSA journals. He was 1182 the Founding Chair of IEEE Workshop on Optical Wireless Communications. 1183

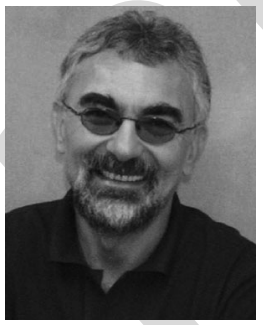

Lajos Hanzo (M'91-SM'92-F'04) received the 1184 D.Sc. degree in electronics and the Doctorate degree 1185 in 1976 and 1983, respectively. In 2009, he was 1186 awarded the honorary doctorate "Doctor Honoris 1187 Causa" by the Technical University of Budapest. 1188 During his 38-year career in telecommunications, 1189 he has held various research and academic posts in 1190 Hungary, Germany, and the U.K. Since 1986, he has 1191 been with the School of Electronics and Computer 1192 Science, University of Southampton, U.K., where he 1193 holds the chair in telecommunications. He has suc- 1194 cessfully supervised about $100 \mathrm{Ph} . \mathrm{D}$. students, coauthored 20 Wiley/IEEE 1195 Press books on mobile radio communications totaling in excess of 100001196 pages, published more than 1400 research entries at IEEE Xplore, acted both 1197 as TPC and General Chair of IEEE conferences, presented keynote lectures 1198 and has been awarded a number of distinctions. Currently, he is directing a 1199 100-strong academic research team, working on a range of research projects 1200 in the field of wireless multimedia communications sponsored by industry, 1201 the Engineering and Physical Sciences Research Council (EPSRC) U.K., the 1202 European Research Council's Advanced Fellow Grant, and the Royal Society's 1203 Wolfson Research Merit Award. He is a Fellow of REng, IET, and EURASIP. 1204 $\mathrm{He}$ is also a Governor of the IEEE VTS. During 2008-2012, he was the Editor- 1205 in-Chief of the IEEE Press and also a Chaired Professor at Tsinghua University, 1206 Beijing. His research is funded by the European Research Council's Senior 1207 Research Fellow Grant. 


\section{QUERIES}

Q1: Please provide post code for the affiliations.

Q2: Please be advised that per instructions from the Communications Society this proof was formatted in Times Roman font and therefore some of the fonts will appear different from the fonts in your originally submitted manuscript. For instance, the math calligraphy font may appear different due to usage of the usepackage[mathcal] \{euscript\}. We are no longer permitted to use Computer Modern fonts. 


\title{
Users First: User-Centric Cluster Formation for Interference-Mitigation in Visible-Light Networks
}

\author{
Xuan Li, Fan Jin, Rong Zhang, Member, IEEE, Jiaheng Wang, Senior Member, IEEE, Zhengyuan Xu, \\ and Lajos Hanzo, Fellow, IEEE
}

\begin{abstract}
Visible light communication (VLC) combined with advanced illumination may be expected to become an integral part of next-generation heterogeneous networks. In order to mitigate the performance degradation imposed by the intercellinterference (ICI), a user-centric (UC) cluster formation technique employing vectored transmission (VT) is proposed for the VLC down-link system, where multiple users may be simultaneously supported by multiple access points (APs). In contrast to the traditional network-centric (NC) design, the UC-VT cluster formation is dynamically constructed and adjusted, rather than remaining static. Furthermore, we consider the critical issue of multiuser scheduling (MUS) relying on maximizing the "sum utility" of this system, which leads to a joint cluster formation and MUS problem. In order to find a practical solution, the original problem is reformulated as a maximum weighted matching (MWM) problem relying on a user-AP distance-based weight and then a low-complexity greedy algorithm is proposed, which offers a suboptimal yet compelling solution operating close to the optimal value found by the potentially excessive-complexity exhaustive search. Our simulation results demonstrate that the proposed greedy MUS algorithm combined with the UC-VT cluster formation is capable of providing an average user throughput of about $90 \%$ of the optimal throughput, which is about three times the throughput provided by the traditional cellular design in some of the scenarios considered.
\end{abstract}

Index Terms-Visible light communication, user-centric cluster formation, multi-user scheduling, maximum weighted matching.

$\mathbf{O}$ WING to its huge unlicensed bandwidth, high data rate potential, energy-efficient illumination etc., the research of Visible Light Communication (VLC) intensified during the past decade or so [1]. As a complementary extension of classic radio frequency communications, extensive investigations have

Manuscript received April 9, 2015; revised July 20, 2015; accepted August 3,2015 . This work was supported by the RC-UK under the auspices of the UKIndia ATC in Wireless Communications, of the Chinese Scholarship Council as well as of the European Research Council's Advanced Fellow Grant. The work of J. Wang was supported in part by the 973 Program of China under Grant 2013CB336600, in part by the NSFC under Grant 61201174, in part by the NSF of Jiangsu under Grant BK2012325, and in part by the Fundamental Research Funds for the Central Universities. The associate editor coordinating the review of this paper and approving it for publication was Dr. Peter Rost.

X. Li, F. Jin, R. Zhang, and L. Hanzo are with the University of Southampton, Southampton, U.K. (e-mail: x126g12@ecs.soton.ac.uk; fj1g10@ ecs.soton.ac.uk; rz@ecs.soton.ac.uk; 1h@ecs.soton.ac.uk).

J. Wang is with the Southeast University, Nanjing, China (e-mail: jhwang@seu.edu.cn).

Z. Xu is with the University of Science and Technology of China, Hefei, China (e-mail: xuzy@ustc.edu.cn).

Color versions of one or more of the figures in this paper are available online at http://ieeexplore.ieee.org.

Digital Object Identifier 10.1109/TWC.2015.2466539 been dedicated to the point-to-point transmission and reception techniques in VLC networks [2]-[10], as also indicated by the IEEE 802.15.7 standard ratified for short-range visible light wireless communication [11]. Apart from their multifold advantages, naturally, VLC systems also exhibit several potential drawbacks, such as reduced performance in non-lineof-sight scenarios, lack of native up-link support, a confined coverage compared to cellular radio frequency networks etc.

Amongst all the design challenges, the performance degradation imposed by Inter-Cell Interference (ICI) at the cell edge may lead to dramatic reduction of the Quality of Service (QoS) in a VLC down-link system. As a result, careful VLC cell formation becomes crucial, since it is the salient design stage of the entire system design cycle. Traditional designs conceived for VLC cells operating both with and without Frequency Reuse (FR) or fractional frequency reuse planning, have been studied in [12]-[14], where each optical Access Point (AP) illuminates a small confined cell. As a further advance, a multi-AP joint transmission scheme relying on Combined Transmission (CT) and Vectored Transmission (VT) ${ }^{1}$ were also investigated in [12]. In contrast to the above-mentioned Network-Centric (NC) design philosophy, a novel User-Centric (UC) cell formation regime was proposed in [15]-[17], where amorphous user-specific multi-AP cells are constructed for jointly transmitting data to a single User Equipment (UE) ${ }^{2}$ by employing $\mathrm{CT}$, which we referred to as UC-CT. By definition, UC design is different from the NC design, where the network configuration is fixed, regardless of the tele-traffic. In order to further improve the achievable bandwidth efficiency of the previously proposed UC-CT and to allow each multi-AP cell simultaneously serve multiple UEs as discussed in [12], we propose the UC-VT-based cluster formation principle in this paper. UC-VT cluster formation may be defined as forming the UC-VT clusters, where each UC-VT cluster is served by a set of VLC APs, which simultaneously serve multiple UEs by employing VT. More explicitly, a UC-VT cluster includes a set of APs and UEs as well as the transmission links between them. Note that the previously proposed UC-CT-based cell formation of [15]-[17]

\footnotetext{
${ }^{1}$ In [12], relying on CT, each individual VLC AP of a multi-AP cell conveyed the same information on the same visible carrier frequency in their overlapping areas and served a single user at a time. In order to eliminate the bandwidth efficiency reduction imposed by CT, Zero-Forcing (ZF)-based VT techniques were employed for serving multiple users at the same time in the overlapping area, which will be exemplified in Section II-C.

${ }^{2} \mathrm{~A}$ single UE represents a communication device equipped with a VLC receiver in our down-link VLC system, which could be a smart phone, a personal computer, a tablet, a printer, etc.
} 
may be regarded as a special case of our UC-VT-based cluster formation, when only a single UE resides within the coverage of the UC-VT cluster.

When multiple UEs are present in a VLC network, efficient resource allocation and Multi-User Scheduling (MUS) constitutes one of the salient problems, which in fact affects all multi-user networks. However, the problem of VLC-based networks has remained to a large extent hitherto unexplored in the open literature, although recently some valuable studies were disseminated in the context of NC single-AP VLC cells [18]-[21]. In particular, the authors of [18] proposed a heuristic scheme for allocating interference-constrained sub-carriers in a multiple access VLC system relying on Discrete MultiTone (DMT) modulation, in order to improve the aggregate throughput. The authors of [19] carefully designed a logical framework aiming to localize, access, schedule and transmit in VLC systems, which was capable of achieving a substantial throughput at a modest complexity. However, similar to most of the literature studying resource allocation in VLC-based systems, both [18] and [19] endeavour to improve the attainable throughput without giving any cognizance to the fairness experienced by the UEs. By taking fairness into account, the authors of [20] proposed an Incremental Scheduling Scheme (ISS), where the global scheduling phase is responsible for assigning the resources to the UEs, while the local scheduling phase regularly adjusts the resource allocation by backtracking the UEs' movements. Furthermore, the authors of [21] proposed a Proportional Fairness (PF) based scheduling algorithm for a centrally controlled VLC system, which outperformed the maximum-rate scheduling policy in terms of balancing the achievable throughput against the fairness experienced by the UEs. Broadly speaking, most studies of the MUS problem encountered in VLC systems are based on single-AP VLC cells. By contrast, we are going to tackle the problems of MUS and UC-based cluster formation relying on VT.

Against the above-mentioned background, in this paper,

i) we investigate the MUS problem relying on the UEs' $\mathrm{PF}$ as a measure by assigning each UE a specific scheduling priority, which is inversely proportional to its anticipated resource consumption [22] and then maximizing a carefully selected network utility function [23], when jointly considering amorphous UC-VT cluster formations for the VLC down-link.

ii) More explicitly, the optimal solution of this joint UCbased cluster formation and MUS problem is first found by a high-complexity exhaustive search, which may have an overwhelming complexity even for a modest-scale system. In order to reduce the computational complexity, the original problem is formulated as a Maximum Weighted Matching (MWM) problem and multiple UEs are scheduled by solving the Kuhn-Munkres (KM) algorithm [24]-[28].

iii) To further improve the grade of practicability, a greedy algorithm is proposed, which operates at a considerably lower complexity, despite taking into account the dynamics of the UC-VT clusters.

iv) Moreover, the computational complexity of both the exhaustive search and of the proposed schemes is analysed and various cluster formations are evaluated for 134 diverse VLC characteristics, such as the Field-Of-View 135 (FOV), the Line-Of-Sight (LOS) blocking probabilities, 136 the optical AP arrangement, etc. 137

VLC can be considered as a new member in the small-cell 138 family of the Heterogeneous Network (HetNets) landscape for 139 complementing the overloaded radio frequency band [17]. The 140 UC cluster formation principle designed for VLC environments 141 constitutes a novel and competitive design paradigm for the 142 super dense multi-tier cell combinations of HetNets, where 143 the sophisticated UEs can actively participate in cell planning, 144 resource management, mobility control, service provision, sig- 145 nal processing, etc. Considering the large-scale multi-input- 146 multi-output systems for example, the antenna selection scheme 147 or beamforming techniques may be designed in a similar UC 148 manner, according to the UEs' geo-location and service require- 149 ments. As a result, the UC concept may be expected to become 150 one of the disruptive techniques to be used in the forthcoming 151 $5 \mathrm{G}$ era [17].

The rest of this paper is organized as follows. Our system 153 model and the UC-VT clusters considered are presented in 154 Section II. Our MUS methodology is described and evaluated 155 in Section III and Section IV, respectively, while dynamically 156 constructing UC-VT clusters. Finally, Section V offers our 157 conclusions.

\section{System MOdEL}

The VLC down-link is considered, which is constituted by a 160 set of VLC APs and each of them relies on an LED array con- 161 structed from several LEDs. The essence of our UC-VT cluster 162 formation is to assign the UEs and optical APs to each other for 163 the sake of maximizing the total utility after employing VT in 164 each of the UC-VT cluster. This procedure is entirely based on 165 the UEs' specific conditions and thus leads to UC clusters. In 166 this section, we first discuss the optical link characteristics and 167 cluster formation, before investigating how to select the UE set 168 supported by a specific AP set in a multi-user system.

\section{A. Link Characteristics}

Since each UE has a limited FOV, they can only receive infor- 171 mation from the optical APs, when one or more APs reside 172 within the UE's FOV. According to [29], if the angle of inci- 173 dence $\psi$ from an AP to a UE is less than the UE's FOV $\psi_{\mathrm{F}}$, the 174 optical channel's Direct Current (DC) attenuation of the LOS 175 path is given by

$$
h_{d}=\frac{(m+1) D_{\mathrm{PA}}}{2 \pi l^{2}} \cos ^{m}(\phi) T_{s}(\psi) g(\psi) \cos (\psi),
$$

where the Lambert index $m$ depends on the semi-angle $\phi_{1 / 2} 177$ at half-illuminance of the source, which is given by $m=178$ $-1 / \log _{2}\left(\cos \phi_{1 / 2}\right)$. Furthermore, $D_{\mathrm{PA}}$ is the physical area of 179 the detector's Photo-Diode (PD), $l$ is the distance between 180 the VLC transmitter and the receiver, while $\phi$ is the angle 181 of irradiance. Still referring to $(1), T_{s}(\psi)$ and $g(\psi)$ denote 182 the gain of the optical filter and of the optical concentrator 183 
TABLE I

VLC PARAMETERS

\begin{tabular}{l|r}
\hline \hline Semi-angle at half power $\left(\phi_{1 / 2}\right)$ & $70^{\circ}$ \\
Modulation bandwidth $(B)[7]$ & $20[\mathrm{MHz}]$ \\
Physical area of a PD $\left(D_{\mathrm{PA}}\right)$ & $1.0\left[\mathrm{~cm}^{2}\right]$ \\
Gain of an optical filter $\left(T_{s}(\psi)\right)$ & 1.0 \\
Refractive index of a lens at a PD $(n)$ & 1.5 \\
O/E conversion efficiency $(\gamma)$ & $0.53[\mathrm{~A} / \mathrm{W}]$ \\
\hline \hline
\end{tabular}

employed, respectively, while $g(\psi)$ can be written as $g(\psi)=$ $n^{2} / \sin ^{2} \psi_{\mathrm{F}}$ [29], where $n$ is the refractive index of a lens at a PD. Furthermore, according to [10], when the incidence angle $\psi$ is no larger than the FOV, the channel's DC attenuation on the first reflection is given by

$$
\begin{aligned}
d h_{r}= & \frac{(m+1) D_{\mathrm{PA}}}{2 \pi^{2} l_{1}^{2} l_{2}^{2}} \rho d D_{\text {wall }} \cos ^{m}(\phi) \cos \left(\beta_{1}\right) \\
& \cos \left(\beta_{2}\right) T_{s}(\psi) g(\psi) \cos (\psi),
\end{aligned}
$$

where $l_{1}$ denotes the distance between an AP and a reflective point, while $l_{2}$ is the distance between this point and a UE. The reflectance factor and the reflective area are denoted by $\rho$ and $d D_{\text {wall }}$, respectively. Additionally, $\beta_{1}$ and $\beta_{2}$ represent the irradiance angles to the reflective point and to the UE, respectively. Our VLC parameter values are summarized in TABLE I.

\section{B. Cluster Formation}

Following the traditional cellular design principle, each optical AP illuminates an individual cell and adopts Unity Frequency Reuse (UFR) across all cells, where the ICI is imposed by the LOS ray of neighbouring cells and consequently the UE may experience dramatic performance degradation at the cell edge. In order to reduce the ICI, appropriate FR patterns may be employed as an appealingly simple solution, while the system has to obey the classic trade-off between reduced bandwidth efficiency and improved cell-edge Signalto-Interference-plus-Noise-Ratio (SINR), when using a FR factor higher than one, as investigated in our previous work [12]. Apart from the single-AP cells, we studied multi-AP merged cells, where several neighbouring VLC APs cooperate by employing either CT or VT techniques. The above-mentioned cell designs, including regular UFR/FR and merged multi-AP cells with CT/VT, rely on a fixed cell-shape, regardless of the traffic requirements, which are referred to as $\mathrm{NC}$ formations. In contrast to the fixed-shape $\mathrm{NC}$ cell formation designs, the UC design philosophy was proposed in [15]-[17], which was capable of supporting irregular-shape elastic cell formations that were capable of accommodating dynamic traffic requirements. By employing CT, each multi-AP UC-CT cell of [15] is only capable of supporting a single UE in a specific time slot. In order to serve multiple UEs at the same time, we propose the VT aided UC cluster formation, which is referred to as a UCVT cluster in this paper. Let us now discuss the model of our system in more detail.

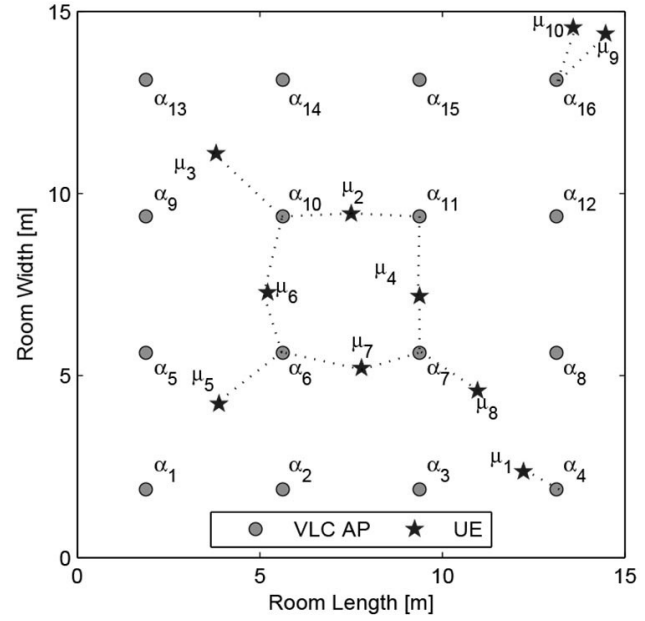

(a)

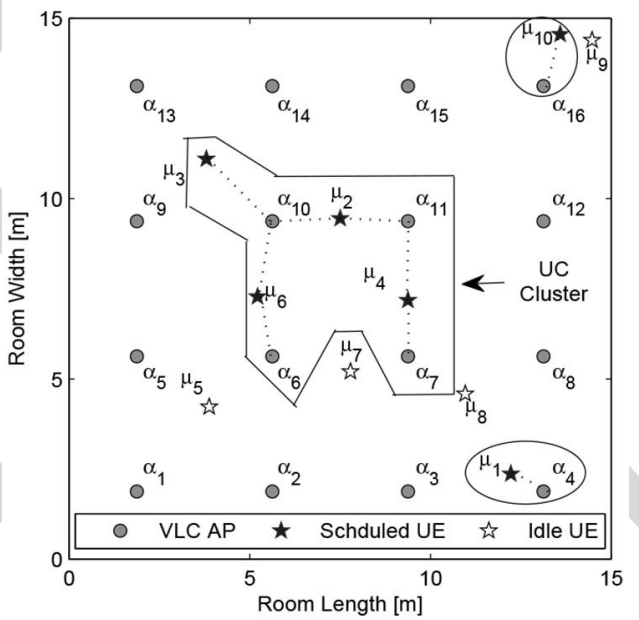

(b)

Fig. 1. (a) Layout of the VLC APs and UEs projected on the horizontal plane, where $\alpha_{i}$ and $\mu_{j}$ represent the VLC APs and UEs, respectively. All LOS links are denoted by dotted lines and for simplicity, the reflections are not shown in this figure. There are $(4 \times 4)=16$ APs and 10 UEs. (b) The cluster formation result provided by Fig. $5 \mathrm{~d}$ for the VLC system of (a).

Fig. 1a shows the example of a particular VLC down-link 223 network having $N_{A}=16$ optical APs and $N_{U}=10 \mathrm{UEs}$, where 224 all LOS links are denoted by dotted lines and for simplicity, 225 the reflections are not shown. Let us first construct the link's 226 bipartite graph $\mathcal{G}(\mathcal{V}, \mathcal{E})$, as shown in Fig. 2a, for the network of 227 Fig. 1a. The vertex set $\mathcal{V}$ denoting the communication nodes is 228 divided into two subsets, i.e. the optical AP set $V_{A}$ as well as 229 the VLC UE set $\mathcal{V}_{U}$, where we have

$$
\begin{aligned}
\mathcal{V} & =\mathcal{V}_{A} \cup \mathcal{V}_{U} \\
& =\left\{\alpha_{i} \mid i=1,2, \ldots, N_{A}\right\} \cup\left\{\mu_{j} \mid j=1,2, \ldots, N_{U}\right\},
\end{aligned}
$$

with $\alpha_{i}$ and $\mu_{j}$ denoting the index of VLC APs and UEs, 231 respectively. Hence, the number of vertices in $\mathcal{G}$ is given by 232 $\left(N_{A}+N_{U}\right)$. Furthermore, when a UE can receive data from an 233 AP, either via the direct LOS path or via the reflected path, a 234 link may be established between them, which is said to be an 235 edge, and these two vertices are said to be adjacent. The edge 236 set $\mathcal{E}$ represents all possible links between APs and UEs with 237 


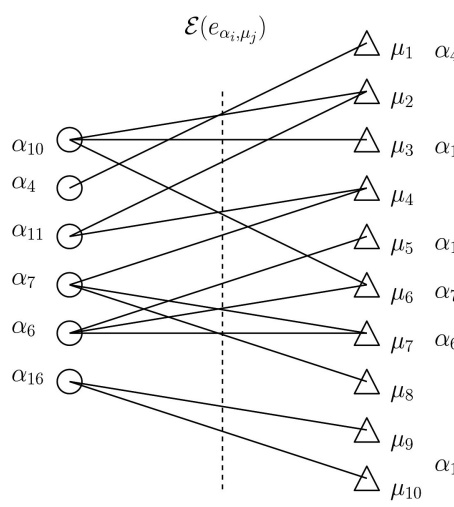

$\operatorname{VLC} \operatorname{AP}\left(\mathcal{V}_{A}\right)$

(a)
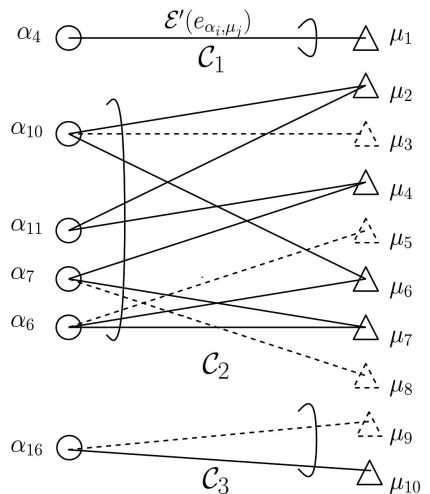

$\mu_{9}$

$\operatorname{VLC}\left(\mathcal{V}_{A}^{\prime}\right)$

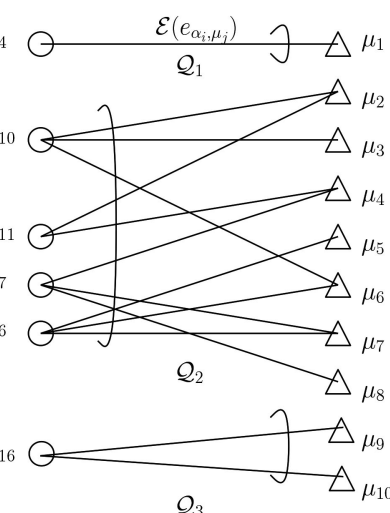

$\mathrm{UC}\left(\mathcal{V}_{U}\right)$

(b)
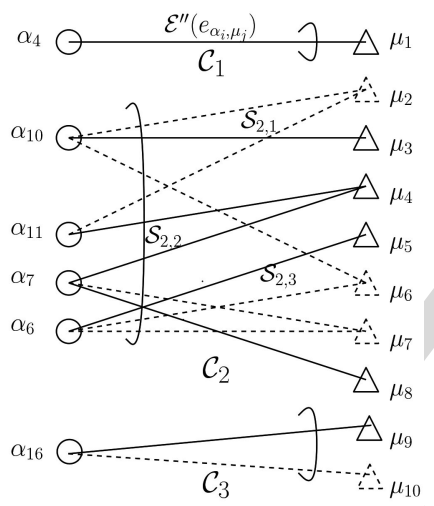

$\mathrm{UE}\left(\mathcal{V}_{U}^{\prime \prime}\right)$

(d)

Fig. 2. (a) A graph model $\mathcal{G}(\mathcal{V}, \mathcal{E})$ of the VLC down-link seen in Fig. 1a. (b) The three independent components of $\mathcal{G}$, i.e. $Q_{1}, Q_{2}$ and $Q_{3}$. (c) and (d) Possible UC-VT cluster formations of the network. In (d), $\mathcal{S}_{2,1}, \mathcal{S}_{2,2}$ and $\mathcal{S}_{2,3}$ are disjoint, but they are regarded as a merged large cluster $\mathcal{C}_{2}$.

one of the endpoints in $\mathcal{V}_{A}$ and the other one in $\mathcal{V}_{U}$, which may be written as

$$
\mathcal{E}=\left\{e_{\alpha_{i}, \mu_{j}} \mid \alpha_{i} \in \mathcal{V}_{A}, \mu_{j} \in \mathcal{V}_{U}\right\},
$$

where $e_{\alpha_{i}, \mu_{j}}$ denotes the link between $\mathrm{AP} \alpha_{i}$ and UE $\mu_{j}$. Since the placement of the VLC APs is fixed, the edge set is determined by the UEs' specific conditions, such as their FOV, position, etc. Therefore, the network graph is said to be UC.

Still referring to Fig. 2a, the graph $\mathcal{G}$ is not fully connected, since not all pairs of vertices are joined by a path. Further scrutiny reveals that $\mathcal{G}$ has three independent components, which are said to be partially connected components, as explicitly shown in Fig. $2 \mathrm{~b}$, marked by $\mathcal{Q}_{1}, \mathcal{Q}_{2}$ and $\mathcal{Q}_{3}$. There are no adjacent AP-UE vertices amongst these distinctive components of $Q_{1}, Q_{2}$ and $Q_{3}$, which indicates that UEs cannot receive data from the optical APs belonging to the other components, only from their own. Thus, the ICI is totally eliminated. Explicitly, since none of the individual components is affected by the others, the proposed cluster formation algorithms may be executed within every single component, as it will be discussed in Section III. On the other hand, in order to simultaneously serve multiple UEs, Zero-Forcing (ZF)-based
VT techniques are introduced in our system. The underlying 258 principle of ZF-based VT is to totally eliminate the interfer- 259 ence at the multiple AP transmitters, so that all the UEs receive 260 mutually interference-free signals. In general, when employing 261 VT the maximum number of UEs supported in a single time 262 slot should be no more than the number of APs. Hence, the ZF- 263 based VT may not be employed directly by each component in 264 Fig. 2b. For example, the number of UEs is almost twice as high 265 as the number of APs in $\mathcal{Q}_{2}$. Therefore, we eliminate the inter- 266 ference by ensuring that only some of the UEs will be scheduled 267 and we solve this problem by constructing a UC-VT cluster 268 with the aid of the serving APs. There are various options for 269 scheduling the UEs shown in Fig. 2c and 2d, where the UEs 270 denoted by the dashed triangle boundary are not scheduled and 271 the edges denoted by dashed lines are not established during 272 the current slot. Furthermore, the UC-VT clusters formed are 273 denoted by $\mathcal{C}_{n}$, i.e. by $\mathcal{C}_{1}, \mathcal{C}_{2}$ and $\mathcal{C}_{3}$ in Fig. 2 c and 2 d. Before 274 investigating how to schedule the UEs, let us first discuss the 275 VT within each UC-VT cluster formed.

\section{Vectored Transmission}

After scheduling the UEs, each UC-VT cluster is formed, as 278 shown for example in Fig. 2c, where the clusters are denoted by 279 $\mathcal{C}_{1}, \mathcal{C}_{2}$ and $\mathcal{C}_{3}$, respectively. Within $\mathcal{C}_{1}$ or $\mathcal{C}_{3}$, only a single UE is 280 supported by a single AP, which is a similar scenario to the reg- 281 ular NC design. However, in order to allow $\left\{\alpha_{10}, \alpha_{11}, \alpha_{7}, \alpha_{6}\right\} 282$ to simultaneously serve all the UEs $\left\{\mu_{2}, \mu_{4}, \mu_{6}, \mu_{7}\right\}$ within 283 $\mathrm{C}_{2}$, we employ Zero-Forcing (ZF)-based VT techniques. More 284 explicitly, we may write the channel's attenuation $\boldsymbol{H}_{\mathrm{C}_{2}}$ between 285 the multiple APs and UEs within $\bigodot_{2}$ as:

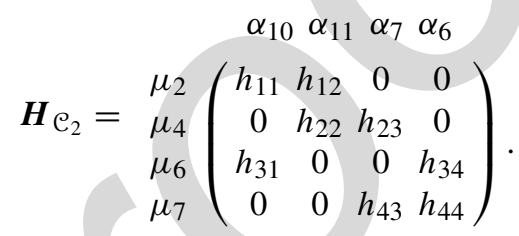

In order to attain mutually interference-free signals at the 287 receivers, the transmitted signals $\boldsymbol{X}_{\mathcal{C}_{2}}=\left[x_{1}, x_{2}, x_{3}, x_{4}\right]$ are 288 precoded as $\left(\boldsymbol{P}_{\mathcal{C}_{2}} \cdot \boldsymbol{X}_{\mathcal{C}_{2}}\right)$ and we may write $\boldsymbol{P}_{\mathcal{C}_{2}}=\left(\boldsymbol{G}_{\mathcal{C}_{2}} \cdot 289\right.$ $\left.\boldsymbol{\Omega}_{\mathcal{C}_{2}}\right)$, where the matrix $\boldsymbol{G}_{\mathcal{C}_{2}}=\boldsymbol{H}_{\mathcal{C}_{2}}^{H} \cdot\left(\boldsymbol{H}_{\mathcal{C}_{2}} \cdot \boldsymbol{H}_{\mathcal{C}_{2}}^{H}\right)^{-1}$ obeys 290 the ZF criterion for the sake of obtaining an interference-free 291 identity matrix for $\boldsymbol{H}_{\mathcal{C}_{2}} \cdot \boldsymbol{G}_{\mathcal{C}_{2}}=\boldsymbol{I}_{4}$ and $\boldsymbol{\Omega}_{\mathcal{C}_{2}}$ is introduced in 292 order to satisfy the power constraint. Hence, the ICI can be 293 totally eliminated at the multiple AP transmitters and as a result, 294 all the UEs receive mutually interference-free signals. Let us 295 now elaborate on the VT techniques a little further in general 296 terms and derive the formations of $\boldsymbol{G}$ and $\boldsymbol{\Omega}$.

Each UC-VT cluster $\mathcal{C}_{n}$ is constituted by a set of APs 298 $\mathcal{V}_{A, \mathcal{C}_{n}}$ with a cardinality of $N_{A, \mathcal{C}_{n}}$ and a set of UEs $\mathcal{V}_{U, \mathcal{C}_{n}} 299$ with a cardinality of $N_{U, \mathcal{C}_{n}}$. Let further $\boldsymbol{X}_{t} \in \mathbb{R}^{N_{U, \mathcal{e}_{n}} \times 1}$ and 300 $\boldsymbol{Y}_{r} \in \mathbb{R}^{N_{U, e_{n} \times 1}}$ denote the vectors of transmitted and received 301 signals, respectively. Upon using VT, we have

$$
\boldsymbol{Y}_{r}=\gamma \cdot P_{t} \cdot \boldsymbol{H} \cdot \boldsymbol{G} \cdot \boldsymbol{\Omega} \cdot \boldsymbol{X}_{t}+\boldsymbol{N},
$$

where $\gamma$ and $P_{t}$ denote the Optical/Electronic (O/E) conver- 303 sion efficiency and the transmitted optical power, respectively. 304 
Furthermore, $\boldsymbol{N}$ denotes the noise, while the channel-matrix $\boldsymbol{H} \in \mathbb{R}^{N_{U}, \mathrm{e}_{n}} \times N_{A, \mathrm{e}_{n}}$ hosts the DC attenuations between the $N_{U, \mathcal{C}_{n}}$ UEs and the $N_{A, \mathfrak{C}_{n}}$ APs, while the matrix $\boldsymbol{G}=\boldsymbol{H}^{H}$. $\left(\boldsymbol{H} \cdot \boldsymbol{H}^{H}\right)^{-1}$ obeys the ZF criterion, which hence results in a beneficial interference-free identity matrix for $\boldsymbol{H} \cdot \boldsymbol{G}=\boldsymbol{I}_{N_{U, \mathrm{e}_{n}}}$. Finally, the matrix $\boldsymbol{\Omega}$ is introduced to enforce the per-AP power constraints, hence we have

$$
\boldsymbol{\Omega}=\varphi \boldsymbol{I}_{N_{U, \mathrm{e}_{n}}}, \quad \varphi=\min _{i=1,2, \ldots, N_{A, \mathrm{e}_{n}}} \sqrt{\frac{1}{\|\boldsymbol{G}(i,:)\|_{F}^{2}}},
$$

where $\boldsymbol{G}(i,:)$ is the $i$ th row of $\boldsymbol{G}$. To elaborate a little further, assuming that we have the per-AP optical power constraint of $P_{t}$, the signal transmitted with the equal power from the $i$ th $\mathrm{AP}$ is $\varphi^{2}\|\boldsymbol{G}(i,:)\|_{F}^{2}$. Note that we have $P_{e}=$ $\pi P_{t}^{2}$, when considering the Asymmetrically-Clipped Optical OFDM (ACO-OFDM) [8]. Hence, we have $\varphi^{2}\|\boldsymbol{G}(i,:)\|_{F}^{2} \leq$ $\pi P_{t}^{2} \Rightarrow \varphi \leq \pi P_{t}^{2} / \sqrt{\|\boldsymbol{G}(i,:)\|_{F}^{2}}$. In order to let each AP satisfy the power constraint, we have $\varphi=\min _{i} \pi P_{t}^{2} / \sqrt{\|\boldsymbol{G}(i,:)\|_{F}^{2}}$, as indicated in (7). Furthermore, let us define the SINR as the aggregate electronic power over the noise power in a bandwidth of $B$ [MHz] [7] plus the sum of the electronic power received from other optical sources in the vicinity. Since the corresponding electronic power is proportional to the square of the electronic current's amplitude and both the intra-cluster and inter-cluster LOS interferences are mitigated, we may express the SINR for a particular UE $\mu_{j}$ within the cluster $\mathcal{C}_{n}$ as

$$
\xi=\frac{\gamma^{2} P_{t}^{2} \varphi^{2} \pi}{N_{0} B+I_{r}}
$$

where $I_{r}$ is the interference imposed by the reflected light. Since the interference power received by the cluster under consideration is influenced by the ZF-based VT within other clusters, for simplicity, we assume that the interference imposed is always equal to its maximum value, which characterizes the worst-case situation in our VT cluster formations. Furthermore, $N_{0}\left[\mathrm{~A}^{2} / \mathrm{Hz}\right]$ is the noise power spectral density dominated by the shot noise $N_{\text {shot }}[10]$ given by $N_{0} \cong N_{\text {shot }}=q I_{a}\left(P_{r}\right) \sim$ $10^{-22}$, where $q$ denotes the electron charge and $I_{a}\left(P_{r}\right)$ is the photo-current at the receiver [7].

Note that there are two popular techniques of constructing white LEDs, namely either by mixing the Red-Green-Blue (RGB) frequencies using three chips, or by using a single blue LED chip with a phosphor layer. We consider the latter one, which is the favoured commercial version. Although the terminology of 'white' LED gives the impression of having all frequency components across the entire visible light spectrum, in fact only the blue frequency-range is detected. More explicitly, not even the entire blue frequency-range is detected, since the less responsive phosphorescent portion of the frequencyband is ignored. Hence, the modulation bandwidth is typically around $20 \mathrm{MHz}$, albeit this measured bandwidth depends on the specific LED product used. Given this $20 \mathrm{MHz}$ bandwidth, we are now ready to employ ACO/DC biased Optical (DCO)-OFDM and partition it into arbitrary frequency reuse patterns.

\section{Methodology}

Let us now schedule multiple UEs in the VLC system in a 355 PF manner by taking into account our UC-VT cluster forma- 356 tion, which is ultimately a joint UC-VT cluster formation and 357 MUS problem. In this section, we commence with a general for- 358 mulation of this joint problem and then propose an exhaustive 359 search method, which finds the optimal solution maximizing 360 the aggregate utility of the VLC system considered. In order 361 to reduce the computational complexity imposed, the original 362 problem is reformulated as an MWM problem, whose optimal 363 solution is provided by the classic KM-algorithm-based [24] 364 approach. For further simplifying the MUS process, we pro- 365 pose a greedy scheduling algorithm for finding a suboptimal 366 solution for our original joint problem, whilst imposing a sig- 367 nificantly reduced complexity. Note that for simplicity, we only 368 consider LOS links in terms of constructing UC-VT clusters. 369 By contrast, in addition to the LOS component, the effect of the 370 first reflection will also be considered, when calculating both 371 the UEs' SINR and the achievable data rate, as indicated in (8). 372 However, our algorithm is a generic one, which may be readily 373 applied, when considering the reflected light for UC-VT cluster 374 formation.

\section{A. Problem Formulation}

Our goal is to find the optimal UC-VT cluster formation for 377 maximizing the long-term network-wide utility, while schedul- 378 ing UEs in a PF manner, which is ultimately a joint cluster 379 formation and MUS problem. In order to implement a PF 380 scheduler, the weight of each link between APs and UEs may 381 be defined as

$$
\omega\left(e_{\alpha_{i}, \mu_{j}}\right)=\frac{r_{\alpha_{i}, \mu_{j}}}{\hat{r}_{\mu_{j}}}, \quad e_{\alpha_{i}, \mu_{j}} \in \mathcal{E},
$$

where $r_{\alpha_{i}, \mu_{j}}$ denotes the achievable data rate of the UE $\mu_{j}$ from 383 the AP $\alpha_{i}$ during the current slot. Since the SINR $\xi$ experienced 384 by a particular UE is determined by the channel attenuation 385 matrix (5) between the APs and UEs within the cluster, $r_{\alpha_{i}, \mu_{j}} 386$ should be a function of the cluster formation, which may be 387 written as:

$$
r_{\alpha_{i}, \mu_{j}}=f\left(\mathcal{E}^{\prime}\right), \quad e_{\alpha_{i}, \mu_{j}} \in \mathcal{E}^{\prime}, \mathcal{E}^{\prime} \subseteq \mathcal{E},
$$

where $\mathcal{E}^{\prime}$ is the set of established links, after the UEs have 389 been scheduled and the UC-VT clusters have been constructed. 390 Furthermore, $\hat{r}_{\mu_{j}}$ denotes the long-term average throughput of 391 the UE $\mu_{j}$, which may be obtained over a time window $T_{F}$ as a 392 moving average according to [30]:

$$
\hat{r}_{\mu_{j}}^{(t)}= \begin{cases}\left(1-\frac{1}{T_{\mathrm{F}}}\right) \hat{r}_{\mu_{j}}^{(t-1)}+\frac{1}{T_{\mathrm{F}}} r_{\alpha_{i}, \mu_{j}}^{(t)}, & \text { if scheduled, } \\ \left(1-\frac{1}{T_{\mathrm{F}}}\right) \hat{r}_{\mu_{j}}^{(t-1)}, & \text { if not scheduled. }\end{cases}
$$

For a given UC-VT cluster formation $\left\{\mathcal{C}_{n}\right\}$, the aggregate util- 394 ity may be formulated by taking into account the weight of 395 
each edge, where again, the weight physically represents the PF scheduling priority of the link [30], which is formulated as:

$$
\begin{aligned}
W & =\sum_{e_{\alpha_{i}, \mu_{j}} \in \mathcal{E}^{\prime}} \omega\left(e_{\alpha_{i}, \mu_{j}}\right) \\
& =\sum_{\alpha_{i} \in \mathcal{V}_{A}^{\prime}} \sum_{\mu_{j} \in \mathcal{V}_{U}^{\prime}} \frac{r_{\alpha_{i}, \mu_{j}}}{\hat{r}_{\mu_{j}}}, \quad \mathcal{E}^{\prime} \subseteq \mathcal{E},
\end{aligned}
$$

where $\mathcal{V}_{A}^{\prime}$ and $\mathcal{V}_{U}^{\prime}$ denote the serving APs and the scheduled UEs set, respectively. It is plausible that various UC-VT cluster formations may lead to different total utility. The maximum value of the aggregate utility $W$ may be achieved by finding the optimal cluster formation. Thus, our problem may be described as selecting an appropriate set of edges $\mathcal{E}^{*}$ from $\mathcal{E}$ and then forming several UC-VT clusters, which maximizes (12). Hence, our Objective Function (OF) may be formulated as:

$$
\mathcal{E}^{*}=\arg \max _{\mathcal{E}^{\prime} \subseteq \mathcal{E}}(W)=\arg \max _{\mathcal{E}^{\prime} \subseteq \mathcal{E}}\left(\sum_{\alpha_{i} \in \mathcal{V}_{A}^{\prime}} \sum_{\mu_{j} \in \mathcal{V}_{U}^{\prime}} \frac{r_{\alpha_{i}, \mu_{j}}}{\hat{r}_{\mu_{j}}}\right) .
$$

Note that in (13) we focus our attention on the aggregate utility of the entire system and do not distinguish, which particular APs and UEs belong to which UC-VT clusters. Let us now discuss the constraint of (13), from the perspective of a single UC-VT cluster. As mentioned in Section II-B, the number of scheduled UEs should not exceed the service capability of a cluster employing VT, where again, the maximum number of UEs supported is equal to the number of APs. Hence, within a single UC-VT cluster $\mathcal{C}_{n}$ we have

$$
N_{A, \mathcal{C}_{n}} \geq N_{U, \mathcal{C}_{n}} .
$$

For solving (13) under the constraint of (14) and finding the optimal cluster formation, we have to know the weight of all edges in $\mathcal{E}$. However, according to (9), the weight $\omega\left(e_{\alpha_{i}, \mu_{j}}\right)$ of a particular link is defined as a function of the data rate achieved by one of its endpoints $\mu_{j}$ during its reception from the other endpoint $\alpha_{i}$, which can only be determined after all clusters have been formed, as briefly introduced in Section II-C. To the best of our knowledge, the optimal solution of this joint problem may only be found via exhaustive search.

\section{B. Optimization of the Joint Problem}

Given a VLC network topology having $N_{A}$ optical APs and $N_{U}$ UEs, it may be composed of some independent components, for example as shown in Fig. 2b. Note that these naturally disjoint components of the network may not constitute the final formations of the UC-VT cluster. More explicitly, there is no limitation concerning the number of APs and UEs within each single component of the network, apart from the fact that within a UC-VT cluster the cardinality of the actively served UE vertex set should be no larger than that of the AP set, as indicated by (14). Each UC-VT cluster should be an independent component of the network, where no ICI is imposed on the neighbouring clusters. Furthermore, each individual network component should be connected at the outset, but each may become disconnected and partitioned into several sub-components/clusters throughout the process of scheduling the UEs, as shown in 439 Fig. 2 d, where $\mathcal{S}_{2,1}, \mathcal{S}_{2,2}$ and $\mathcal{S}_{2,3}$ will be regarded as a large 440 merged cluster.

Still referring to Fig. 2b, in order to find the optimal cluster 442 formation for maximizing (12), the optimization is performed 443 separately in $\mathcal{Q}_{1}, \mathcal{Q}_{2}$ and $\mathcal{Q}_{3}$, which are independent network 444 components. Within $\mathcal{Q}_{1}$, only a single UE $\mu_{1}$ is capable of con- 445 necting with the AP $\alpha_{4}$, where $\alpha_{4}$ either supports $\mu_{1}$ or it will be 446 turned off. Therefore, there are two AP-UE combination scenar- 447 ios for $\mathcal{Q}_{1}$. Within $\mathcal{Q}_{2}$, there are three UEs, i.e. $\mu_{2}, \mu_{3}$ and $\mu_{6}, 448$ which are within the coverage of the AP $\alpha_{10}$. Hence, $\alpha_{10}$ may 449 either select one of them to support or become inactive. Thus, 450 there are $(3+1)$ choices for $\alpha_{10}$. Similarly, the other APs $\alpha_{11}, 451$ $\alpha_{7}$ and $\alpha_{6}$ have $(2+1),(3+1)$ and $(3+1)$ choices, respec- 452 tively. Therefore, the number of possible AP-UE combinations 453 within $Q_{2}$ is $(4 \times 3 \times 4 \times 4=192)$. $Q_{3}$ has an easier situation, 454 where the AP $\alpha_{16}$ may either select one UE from $\left\{\mu_{9}, \mu_{10}\right\} \quad 455$ or opts for providing no services. For the entire network of 456 Fig. 2b, the number of possible AP-UE combinations becomes 457 $((2-1)+(192-1)+(3-1)=194)$. Finally, we take into 458 account the undesired scenario, where all APs are out of service 459 by subtracting 1 . Generally speaking, our exhaustive search- 460 based approach of finding the optimal UC-VT cluster formation 461 is detailed below.

i) For each separate network component $Q_{m}$ relying on 463 $N_{A, Q_{m}}$ APs and $N_{U, Q_{m}}$ UEs, let $N_{U, Q_{m}}^{\alpha_{i}}$ denote the num- 464 ber of possible links between a certain AP $\alpha_{i}$ with the UEs 465 within its coverage, where $i=1,2, \ldots, N_{A, Q_{m}}$. 466

ii) Note that not the entire set of APs has to be active during 467 the scheduling process. In other words, we do not limit the 468 number of active APs or scheduled UEs, when aiming for 469 finding the optimal cluster formation. Thus the concept 470 of a virtual link is introduced for each AP, which theo- 471 retically exists, but it is turned off. Hence, the number of 472 possible AP-UE combinations in $Q_{m}$ may be expressed as 473

$$
\prod_{i=1}^{N_{A, Q_{m}}}\left(N_{U, Q_{m}}^{\alpha_{i}}+1\right)-1
$$

where we have $1 \leq N_{U, Q_{m}}^{\alpha_{i}} \leq N_{U, Q_{m}}$. Note that in (15), 474 subtracting 1 implies that we have removed the undesired 475 scenario, where all APs are turned off. 476

iii) For each possible UC-VT cluster formation in $Q_{m}$, the 477 aggregate utility can be calculated and the optimal for- 478 mation associated with the maximum utility is found 479 correspondingly. Since each network component $Q_{m}$ is 480 independent, the optimal cluster formation is separately 481 found in each of them. Hence, for finding the optimal 482 solution of (13) for the entire system, we need to repeat 483 the process of ii) in each $Q_{m}$. Thus the total number of 484 possible AP-UE combinations is the summation of (15) 485 for each $Q_{m}$, which may be expressed as

$$
\sum_{m}\left(\prod_{i=1}^{N_{A, Q_{m}}}\left(N_{U, Q_{m}}^{\alpha_{i}}+1\right)-1\right)
$$

The number of all possible cluster formations within a single 487 scheduling time slot at a ms-based scale is given by (16), which 488 
is jointly determined by the number of APs $\left(N_{A, Q_{m}}\right)$ and number of UEs $\left(N_{U, Q_{m}}\right)$ as well as by the specific distribution of the UEs $\left(N_{U, Q_{m}}^{\alpha_{i}}\right)$. For a network associated with a low density of UEs and a small number of APs, a desirable cluster formation solution may be achieved by exhaustively searching all the possibilities. For example, when 16 APs support 10 UEs, the optimal association will be found after searching $\sim 10^{4}$ possible cluster formations. However, this search-space may become excessive within a time slot at a ms-based scale even for a modest-scale network, which makes the exhaustive search strategy unacceptable owing to its computational complexity. For example, as many as $\sim 10^{7}$ cluster formations have to be searched within a single processing time slot, when there are 20 UEs supported by 16 APs. Hence, instead of solving the joint problem directly, we update the definition of the weight for each link and reformulate the original problem with the goal of significantly reducing the complexity, as it will be detailed in Section IV.

\section{Distance-based Weight and Problem Reformulation}

In (9), the weight of each link is related to the UE's achievable data rate, which cannot be determined before the UC-VT clusters have finally been constructed. Our ultimate goal is that of finding the optimal cluster formation based on the sum weight attained by appropriately scheduling the UEs, as indicated by (13). In other words, the cluster formation and MUS problems were originally coupled. Hence, we opt for simplifying the original problem by adopting a deterministic weight for each AP-UE link. Thus, the maximization of the sum weight may be realized before the UC-VT clusters are constructed, and as a benefit, the joint cluster formation and MUS problem becomes decoupled.

As mentioned in Section III-B, the weight of each link between the AP and the UE is non-deterministic, which is influenced by how the UC-VT clusters are constructed, while the optimal cluster formation solution is determined by maximizing the sum weight of all the scheduled links. Hence, we opt for bypassing the non-deterministic weight assignment and instead, we opt for selecting active links according to their optical channel quality, which is significantly affected by the UE's position, according to (1). We directly adopt each UE's position information for determining the weight of each link and introduce a new weighted bipartite graph $\mathcal{G}_{d}(\mathcal{V}, \mathcal{E})$, which is constructed based on the original graph $\mathcal{G}(\mathcal{V}, \mathcal{E})$ and they have the same vertex and edge sets. However, the weight of each edge is redefined as

$$
\omega_{d}\left(e_{\alpha_{i}, \mu_{j}}\right)=\frac{1 / l_{\alpha_{i}, \mu_{j}}^{3}}{\hat{r}_{\mu_{j}}}, \quad e_{\alpha_{i}, \mu_{j}} \in \mathcal{E},
$$

where $l_{\alpha_{i}, \mu_{j}}$ represents the distance between the AP $\alpha_{i}$ and the $\mathrm{UE} \mu_{j}$. Given that the APs are fixed, the weight is determined by the specific position of each $\mathrm{UE} \mu_{j}$. It can be readily seen from (1) that the VLC links having a shorter length have a better channel quality. Therefore, the weight is inversely proportional to the distance and thus the links associated with better channels have a higher weight. Note that if the UE $\mu_{j}$ is too far away from the AP $\alpha_{i}$, namely $\mu_{j}$ is not within the coverage of $\alpha_{i}$, it is reasonable to assume having $\omega_{d}\left(e_{\alpha_{i}, \mu_{j}}\right)=0$.
Our problem becomes that of selecting a subset of links $\mathcal{E}_{d}^{*} 542$ having a better channel quality, and along with their endpoints 543 they represent our UC-VT cluster formation. In general, within 544 a UC-VT cluster, multiple APs serve multiple UEs and there 545 may not be a one-to-one relationship. Nonetheless, in the first 546 MUS step, we could select the one-to-one AP-UE pairs accord- 547 ing to their distance-based weight, where the serving APs and 548 the scheduled UEs are determined. Then, in the cluster for- 549 mation step, the cluster may be constructed by adding other 550 possible links between the selected AP-UE set. Thus the MUS 551 and cluster formation problem is decoupled and solved sepa- 552 rately. Note that in the MUS step, a specific set of the links 553 between all the AP-UE pairs, which do not share the same AP 554 or UE, is said to represent independent edges and they con- 555 stitute a matching $\mathcal{M}$ defined over the graph. For example, in 556 Fig. 2b we have $6 \mathrm{AP}$ vertices plus $10 \mathrm{UE}$ vertices as well as 557 14 edges. In order to construct a matching, 6 UEs are selected 558 and each of them matches a specific AP associated with one 559 edge, e.g. $\left\{\alpha_{4} \rightarrow \mu_{1}, \alpha_{10} \rightarrow \mu_{3}, \alpha_{11} \rightarrow \mu_{4}, \alpha_{7} \rightarrow \mu_{7}, \alpha_{6} \rightarrow 560\right.$ $\left.\mu_{6}, \alpha_{16} \rightarrow \mu_{9}\right\}$. Furthermore, we have $\mathcal{M} \subseteq \mathcal{E}_{d}^{*} \subseteq \mathcal{E}$. To elabo- 561 rate a litter further in general terms, let us first formally define 562 the matching over a graph. As mentioned in Section III-B, the 563 network graph model may be disconnected and divided into 564 multiple independent components. For an individual compo- 565 nent, denoted by $\mathcal{Q}_{m}\left(\mathcal{V}_{\mathcal{Q}_{m}}, \mathcal{E}_{\mathcal{Q}_{m}}\right)$, which is a subgraph of $\mathcal{G}_{d} 566$ associated with the vertex set $\mathcal{V}_{Q_{m}}$ and the edge set $\mathcal{E}_{Q_{m}}$, a 567 matching $\mathcal{M}_{Q_{m}}$ may be defined as a specific subset of the edge 568 set $\mathcal{E}_{Q_{m}}$, where no pair of edges shares a vertex within $\mathcal{M}_{Q_{m}}$. 569 It is plausible that the cardinality of the edge-subset $\mathcal{M}_{Q_{m}}$ is 570 given by the number of the $\mathcal{M}_{Q_{m}}$-saturated AP/UE vertices, 571 which belongs to the edges of $\mathcal{M}_{Q_{m}}$. Otherwise, the vertices not 572 belonging to the edges of $\mathcal{M}_{Q_{m}}$ are said to be $\mathcal{M}_{Q_{m}}$-unsaturated. 573 Hence, if we allow as many UEs as possible to be scheduled, $\mathcal{M} 574$ should have the highest possible cardinality. Furthermore, con- 575 sidering the weight of each edge, our cluster formation problem 576 may be further reformulated as a MWM problem, where the OF 577 may be written as:

$$
\begin{aligned}
\mathcal{M}_{Q_{m}}^{*} & =\arg \max _{\mathcal{M}_{Q_{m}}}\left(W_{Q_{m}}\right) \\
& =\arg \max _{\mathcal{M}_{Q_{m}}}\left(\sum_{\alpha_{i} \in \mathcal{V}_{Q_{m}}, \mu_{j} \in \mathcal{V}_{Q_{m}}} \omega_{d}\left(e_{\alpha_{i}, \mu_{j}}\right)\right) .
\end{aligned}
$$

Upon solving (18) within each individual network compo- 579 nent, a set of APs as well as UEs is selected in order to form 580 a UC-VT cluster along with all links between them. Thus, the 581 solution of the MWM problem is expected to provide a sub- 582 optimal result for our original joint MUS and UC-VT cluster 583 formation problem, which is however found at a significantly 584 reduced complexity.

\section{Optimal MWM}

If we construct a $\left(N_{A, Q_{m}} \times N_{U, Q_{m}}\right)$-element weight matrix 587 $\left(\omega_{d}\left(e_{\alpha_{i}, \mu_{j}}\right)\right)$ for each of the individual component $Q_{m}$, the prob- 588 lem of (18) may be viewed as being equivalent to finding a set 589 of independent elements from $\left(\omega_{d}\left(e_{\alpha_{i}, \mu_{j}}\right)\right)$, in order to maxi- 590 mize the sum of these elements. The definition of independent 591 


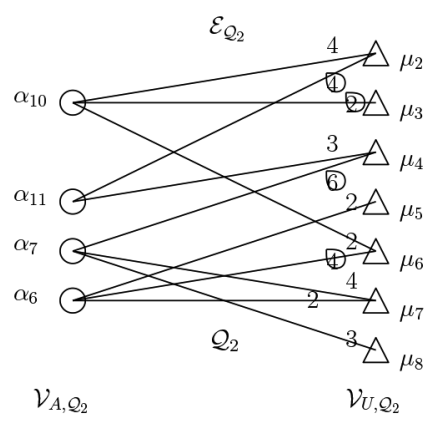

(a)

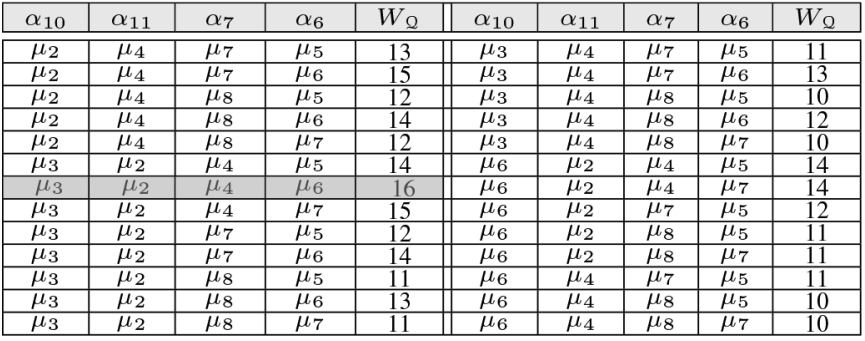

(b)

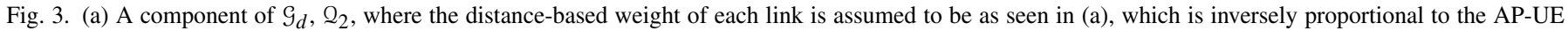

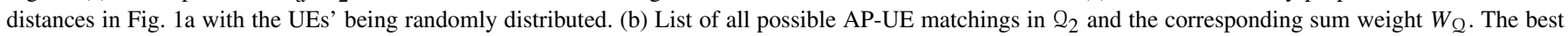
matching associated with the circled weights of (a) is the one in the grey-shaded line 7.

elements indicates that none of them occupies the same row or column, where a row represents an AP and a column represents a UE. To be more explicit, the selected set of the independent elements in the weight matrix corresponds to a matching of the graph, since a single element represents an edge of the graph and no pair of these elements shares the same AP or UE. Thus our MWM problem has also been interpreted in a matrix form. Before finding the optimal solution of the afore-mentioned MWM problem, let us first introduce Theorem 1.

Theorem 1: Given the $\left(n_{r} \times n_{r}\right)$-element matrix $\left(a_{i j}\right)$ and $\left(b_{i j}\right)$, as well as the column vector $\left(c_{i}\right)$ and the row vector $\left(r_{j}\right)$, satisfying $b_{i j}=c_{i}+r_{j}-a_{i j}$, provided the permutation $p\left(p_{i}\right.$ : $\left.i=1, \ldots, n_{r}\right)$ of the integers $1, \ldots, n_{r}$ minimizes $\sum_{i=1}^{n_{r}} a_{i p_{i}}$, $p$ then also maximizes $\sum_{i=1}^{n_{r}} b_{i p_{i}}$.

Proof: Let $p$ be a permutation of the integers $1,2, \ldots, n$ minimizing $\sum_{i=1}^{n_{r}} a_{i p_{i}}$, then we have

$$
\sum_{i=1}^{n_{r}} b_{i p_{i}}=\sum_{i=1}^{n_{r}} c_{i}+\sum_{i=1}^{n_{r}} r_{p_{i}}-\sum_{i=1}^{n_{r}} a_{i p_{i}}
$$

Since the first two terms are constant and independent of $p, \sum_{i=1}^{n_{r}} b_{i p_{i}}$ is maximized, when $\sum_{i=1}^{n_{r}} a_{i p_{i}}$ is minimized by $p$.

Hence, if we want to find the optimal assignment solution for maximizing $\sum_{i=1}^{n} b_{i, p_{i}}$, what we have to do is to transform $\left(b_{i j}\right)$ into $\left(a_{i j}\right)$ as mentioned above and then find the optimal solution minimizing $\sum_{i=1}^{n} a_{i, p_{i}}$, where $\left(a_{i j}\right)$ and $\left(b_{i j}\right)$ are said to be equivalent. For a rectangular $\left(n_{r} \times n_{c}\right)$-element matrix $\left(a_{i j}^{\prime}\right)$, we can obtain a square matrix $\left(a_{i j}\right)$ by attaching $\mid n_{r}-$ $n_{c} \mid$ lines of zero elements to $\left(a_{i j}^{\prime}\right)$. Thus, $\left(a_{i j}^{\prime}\right)$ and $\left(a_{i j}\right)$ have the same optimal assignment solution and Theorem 1 can be readily applied for non-square rectangular matrices, where we have $n_{r} \neq n_{c}$.

In order to solve our MWM problem, which is derived from our joint cluster formation and MUS problem, we introduce the classic Kuhn-Munkres (KM) algorithm [24], [25], which is an efficient method of solving the matching problems of bipartite graphs and may be readily applied in a symmetric graph. However, the number of VLC UEs is usually higher than that of the optical APs within a single network component $Q_{m}$, which results in an asymmetric bipartite graph. Owing to the efforts of
Bourgeois and Lassalle [26], an extension of the KM algorithm 627 was developed for non-square rectangular matrices. Relying on 628 this approach, we introduce a KM-algorithm-based technique 629 of solving our UC-VT cluster formation problem. The mathe- 630 matical formulation of the extended KM algorithm of [26] may 631 be described as that of finding a set of $k$ independent elements 632 $k=\min \left\{n_{r}, n_{c}\right\}$ from a given $\left(n_{r} \times n_{c}\right)$-element matrix $\left(b_{i, j}\right), 633$ in order to minimize the sum of these elements. However, our 634 problem is not a minimization, but a maximization problem 635 associated with the OF of (18). Therefore, we first transform our 636 MWM problem into an equivalent assignment problem based 637 upon Theorem 1 and then invoke the KM algorithm for finding 638 the optimal solution of the equivalent problem, which is also 639 optimal for our MWM problem. Furthermore, since the MWM 640 result of each naturally disjoint network component is mutually 641 independent, the matching algorithm is executed within each 642 individual component in a parallel manner.

As shown in Fig. 3a, $\mathcal{Q}_{2}$ is an independent network compo- 644 nent and also a subgraph of our weighted graph $\mathcal{G}_{d}$, which also 645 shows the individual weights of the $\left\{\alpha_{i}-\mu_{j}\right\}$ links. In order 646 to schedule the maximum number of UEs, given the four APs 647 in $Q_{2}$, four of them will be selected and each one is paired 648 with a specific AP, where the possible matchings and the corre- 649 sponding sum weight values are shown in Fig. 3b. For example, 650 bearing in mind Fig. 3a, the first matching of the first row in 651 Fig. 3b may represent $\left\{\alpha_{10} \rightarrow \mu_{2}, \alpha_{11} \rightarrow \mu_{4}, \alpha_{7} \rightarrow \mu_{7}, \alpha_{6} \rightarrow 652\right.$ $\left.\mu_{5}\right\}$, which leads to a sum weight of $W_{Q}=\sum_{l=1}^{4} \omega_{l}=4+653$ $3+4+2=13$. The specific matching of the seventh row in 654 Fig. $3 \mathrm{~b}$ is $\left\{\alpha_{10} \rightarrow \mu_{3}, \alpha_{11} \rightarrow \mu_{2}, \alpha_{7} \rightarrow \mu_{4}, \alpha_{6} \rightarrow \mu_{6}\right\}$, which 655 is represented by the shaded row of Fig. 3b. This achieves the 656 largest sum weight of $W_{Q}=\sum_{l=1}^{4} \omega_{l}=2+4+6+4=16.657$ The corresponding weights in Fig. 3a are circled. Hence they 658 represent the optimal matching in the scenario considered. 659

Instead of listing all matchings, we now proceed by con- 660 structing an equivalent minimization problem for our MWM 661 and invoke the KM algorithm [24], [26] for finding the opti- 662 mal solution, which is described in detail in Appendix A. 663 As shown in Fig. 4a, the KM-algorithm-based approach pro- 664 vides the optimal solution for the MWM problem (18), with 665 its UE-AP distance-based weight defined by (17). The matched 666 AP-UE pairs form a UC-VT cluster and the aggregate util- 667 ity in (12) can be calculated according to the matching result. 668 


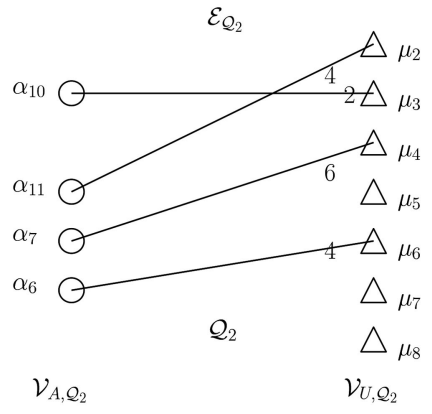

(a)

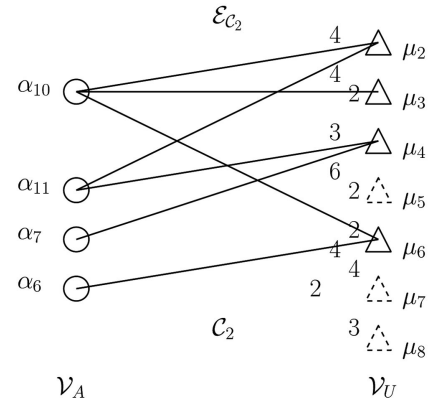

(b)

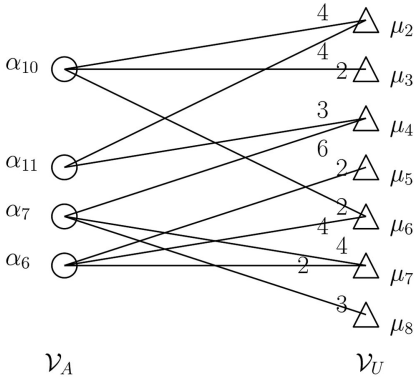

(a)

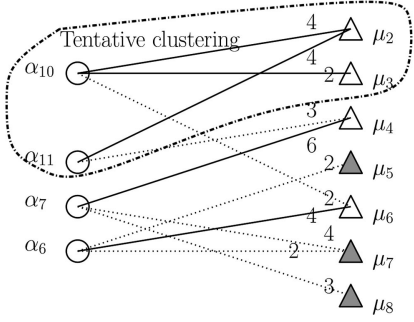

$\mathcal{V}_{A}$

(c)

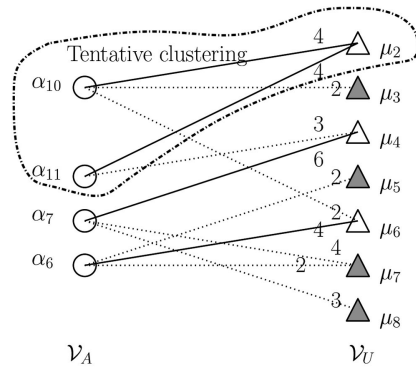

(b)

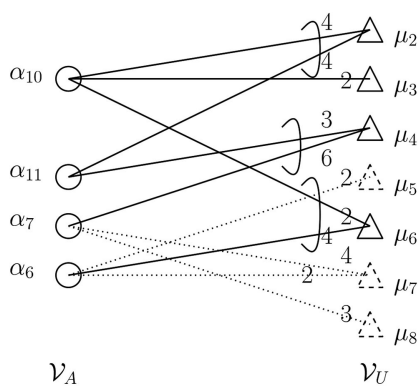

(d)
Fig. 4. (a) The optimal solution for the MWM problem (18) relying on the distance-based weight defined by (17), which is provided by the KM-algorithmbased assignment. (b) The UC-VT cluster formation based on the matching result of Fig. 3a, where more links are added for employing VT and thus the multiple APs $\left\{\alpha_{10}, \alpha_{11}, \alpha_{7}, \alpha_{6}\right\}$ are capable of supporting all the scheduled UEs $\left\{\mu_{2}, \mu_{3}, \mu_{4}, \mu_{6}\right\}$ simultaneously. The triangle with a dashed boundary denotes the specific UE, which is not scheduled during the current slot.

However, by employing VT among the set of APs and UEs, the actual cluster may be formed with the aid of more links, as seen in Fig. 4b. Thus, the UC-VT cluster formation provided by the single-to-single matching solution may not be optimal for (13), but it is capable of offering an acceptable suboptimal solution attained at a lower complexity than that of the exhaustive search. Explicitly, it has a complexity order of $O\left(k^{2} \times l\right)$ [26], where we have $k=\min \left\{N_{A, Q_{m}}, N_{U, Q_{m}}\right\}$ and $l=\max \left\{N_{A, Q_{m}}, N_{U, Q_{m}}\right\}$. The complexity of both the exhaustive search and KM algorithm will be investigated in Section IV in the context of our VLC-based network.

\section{E. Proposed Greedy Cluster Formation/MUS Algorithm}

In order to further simplify the procedures of scheduling the UEs in our UC-VT cluster formation, in this section we propose a greedy cluster formation/MUS algorithm operating at a low complexity, which is also capable of achieving a near-optimal solution for our original cluster formation problem of (13). Before discussing our proposed MUS problem, let us first introduce some notations. Explicitly, $\nu_{U, \alpha_{i}}$ denotes the set of UEs within the coverage of a specific AP $\alpha_{i}$ with a UE-cardinality of $N_{U, \alpha_{i}}$. Each UE $\mu_{j}$ is assumed to have a scheduling priority corresponding to each AP $\alpha_{i}$, which is given by the weight in (17). Let $\mathcal{P}_{\alpha_{i}}=\left(\omega_{d}\left(e_{\alpha_{i}, \mu_{j}}: \mu_{j} \in \mathcal{V}_{U, \alpha_{i}}\right)\right.$ denote the priority of each element of $\mathcal{V}_{U, \alpha_{i}}$ representing the AP $\alpha_{i}$. Furthermore, if a UE does not receive any connection request from any AP during the slot considered, it is said to be an idle UE; otherwise, it is an active UE. Let us now introduce our algorithm by considering Fig. 5a, for example.

i) Initial selection. Each VLC AP $\alpha_{i}$ selects the specific UE $\mu_{j}^{\alpha_{i}}$ from $\nu_{U, \alpha_{i}}$ associated with the highest distance-based priority, which satisfies

$$
\mu_{j}^{\alpha_{i}}=\arg \max _{\mu_{j} \in \mathcal{V}_{U, \alpha_{i}}}\left(\mathcal{P}_{\alpha_{i}}\right)
$$

If the UE $\mu_{j}^{\alpha_{i}}$ receives an assignment request exclusively from the $\mathrm{AP} \alpha_{i}$, this AP-UE pair is referred to
Fig. 5. (a) The network component considered. (b) Initial selection and tentative-cluster construction. The shaded triangles indicate the hitherto unsupported UEs. (c) Expansion of the tentative-cluster. (d) UC-VT cluster formation, where the incomplete ellipsoids indicate the specific UC-VT cooperation requests of the UEs and the finally unscheduled UEs are denoted by the triangles with dashed boundary.

as a Single-to-Single Matching (SSM), which may be 702 formally defined as

$$
\mathcal{M}_{\mathrm{SSM}}=\left\{\alpha_{i} \rightarrow \mu_{j}^{\alpha_{i}}: \forall \alpha_{i^{\prime}} \neq \alpha_{i} \Rightarrow \mu_{j}^{\alpha_{i^{\prime}}} \neq \mu_{j}^{\alpha_{i}}\right\}
$$

For example, as shown in Fig. 5b, $\mu_{4}$ only receives an 704 assignment request from $\alpha_{7}$, although it also falls within 705 the coverage of $\alpha_{11}$, since $\mu_{2}$ has the largest schedul- 706 ing weight of 4 for $\alpha_{11}$ and therefore the $\left\{\alpha_{11} \rightarrow \mu_{4}\right\} \quad 707$ link of weight 3 is ignored. Similarly the $\left\{\alpha_{6} \rightarrow \mu_{6}\right\}$ link 708 of weight 4 is also a SSM, because the $\left\{\alpha_{6} \rightarrow \mu_{5}\right\}$ and 709 $\left\{\alpha_{6} \rightarrow \mu_{7}\right\}$ links have a lower weight of 2 . Hence, the 710 AP-UE association after this initial selection is shown in 711 Fig. 5b, where the low-weight links are only shown with 712 dotted lines.

ii) Tentative-cluster construction. If a UE is offered multiple 714 connection opportunities by different APs, this is said to 715 be a Multiple-to-Single Matching (MSM), which may be 716 defined as

$$
\begin{array}{r}
\mathcal{M}_{\mathrm{MSM}}=\left\{\left(\alpha_{i}, \alpha_{i^{\prime}}, \alpha_{i^{\prime \prime}}, \ldots\right) \rightarrow \mu_{j}^{\alpha_{i}}:\right. \\
\left.\mu_{j}^{\alpha_{i}}=\mu_{j}^{\alpha_{i^{\prime}}}=\mu_{j}^{\alpha_{i^{\prime \prime}}}=\ldots\right\},
\end{array}
$$

where we have $\mathcal{M}_{\mathrm{MSM}}=\left\{\left(\alpha_{10}, \alpha_{11}\right) \rightarrow \mu_{2}\right\}$ in the exam- 718 ple of Fig. 5b, since $\mu_{2}$ has the highest priority for both 719 $\alpha_{10}$ and $\alpha_{11}$. Furthermore, each MSM is assumed to con- 720 struct a tentative-cluster, as also shown in Fig. 5b, where 721 the shaded triangles indicate the hitherto unsupported 722 UEs.

iii) Expansion of the tentative-cluster. Within a tentative- 724 cluster $\left(\alpha_{i}, \alpha_{i^{\prime}}, \alpha_{i^{\prime \prime}}, \ldots\right) \rightarrow \mu_{j}^{\alpha_{i}}$, each AP $\alpha_{i}$ reselects a 725 
hitherto unsupported UE to be supported with the highest priority, provided that there are still unsupported UEs in $\mathcal{V}_{U, \alpha_{i}}$. Accordingly, as indicated by Fig. $5 \mathrm{c}, \alpha_{10}$ reselects the unsupported UE $\mu_{3}$, since the set $\mathcal{V}_{U}^{\alpha_{10}} \backslash\left(\mu_{2}, \mu_{6}\right)=$ $\mu_{3}$ is non-empty and $\mu_{3}$ is the only unsupported UE within the coverage of $\alpha_{10}$. However, since the set $\mathcal{V}_{U}^{\alpha_{11}} \backslash$ $\left(\mu_{2}, \mu_{4}\right)=\emptyset$ is empty, $\alpha_{11}$ does not have any additional UE to support.

iv) Cluster formation. In order to mitigate the inter-cluster interference, the scheduled UEs found in the overlapping areas of some neighbouring APs determine the cooperation of these APs. More explicitly, if a particular scheduled UE has the benefit of a LOS ray from several different APs, then the UE sends a cooperation request to these APs. For example, in Fig. 5d $\mu_{2}$ sends its cooperation request to $\left\{\alpha_{10}, \alpha_{11}\right\}$, while $\mu_{4}$ and $\mu_{6}$ request cooperation with $\left\{\alpha_{11}, \alpha_{7}\right\}$ and $\left\{\alpha_{10}, \alpha_{6}\right\}$, respectively, as indicated by the incomplete ellipsoids. Thus all the cooperating APs and their matching UEs construct a single UC-VT cluster in the examples of Fig. $1 b$.

Recall that $N_{A}$ APs are only capable of simultaneously supporting at most the same number of UEs according to (14). Therefore, during the expansion of the tentative-cluster, the number of active UEs becomes $\left(N_{A}+1\right)$, provided that all APs can connect with an idle UE. Hence, the UE having the smallest priority is removed. Let us now provide an overview of the greedy cluster formation/MUS technique in form of Algorithm 1.

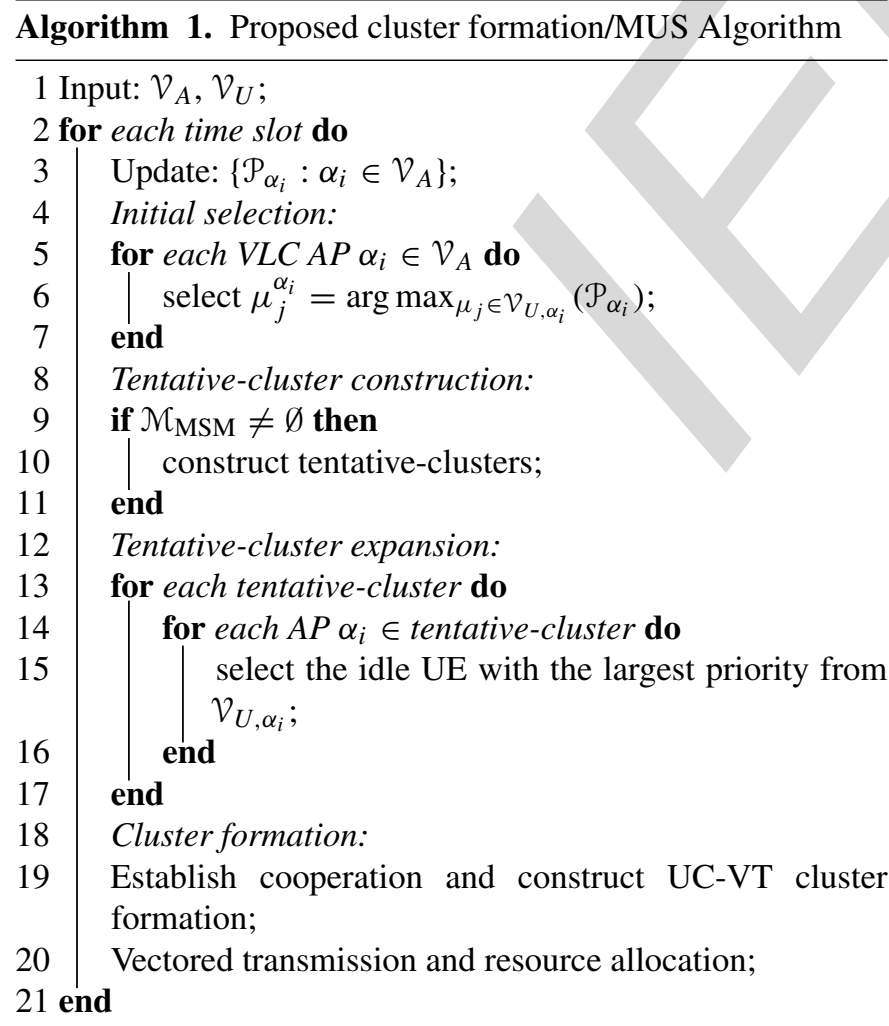

\section{Performance Evaluation}

In this section, we will present our simulation results characterising the MUS and cluster formation algorithms,
TABLE II

SIMULATION PARAMETERS

\begin{tabular}{l|r}
\hline \hline Transmitted optical power per LED lamp $\left(P_{t}\right):$ & $20[\mathrm{~W}]$ \\
$20 \times 20$ LEDs with 50mW per LED & $55^{\circ} / 57.5^{\circ} / 60^{\circ} / 62.5^{\circ}$ \\
Half of the receiver's FOV $\left(\psi_{\mathrm{F}}\right)$ & 0.8 \\
Reflectance factor $(\rho)$ & $14.4[\mathrm{~W}]$ \\
$\begin{array}{l}\text { Power of circled-LED lamp: } 17 \times 17 \text { LEDs } \\
\text { with 50mW per LED }\end{array}$ & $36.8[\mathrm{~W}]$ \\
$\begin{array}{l}\text { Power of cornered-LED lamp: } 23 \times 32 \text { LEDs } \\
\text { with 50mW per LED }\end{array}$ & \\
\hline \hline
\end{tabular}

with a special emphasis on our UC-VT cluster formation. A 781 $15 \mathrm{~m} \times 15 \mathrm{~m} \times 3 \mathrm{~m}$ room model is considered, which is covered 782 by a VLC down-link including $(4 \times 4)$ uniformly distributed 783 optical APs at a height of $2.5 \mathrm{~m}$. The parameters of the 784 LED arrays are summarized in TABLE II. Our investigations 785 include both the LOS and the first reflected light-path, where 786 the channel's DC attenuation is given by (1) and (2), respec- 787 tively. Furthermore, as mentioned in Section II, ACO-OFDM 788 is considered and the associated capacity is given as $R=789$ $\frac{B}{4} \log _{2}(1+\xi)$ according to [9], where $\xi$ is the SINR of (8). Our 790 simulation results were averaged over 50 independent snap- 791 shots and each snapshot is constituted by 50 consecutive time 792 slots having a length of $1 \mathrm{~ms}$. The UEs at a height of $2.5 \mathrm{~m}$ are 793 random uniformly distributed at the beginning of each snapshot 794 and they move randomly during the consecutive 50 time slots 795 at a speed of $1 \mathrm{~m} / \mathrm{s}$. The locations of the UEs are reported every 796 time slot, i.e. every $1 \mathrm{~ms}$.

\section{A. Complexity Analysis}

As shown in Fig. 6a, when the number of UEs is less than 799 5 , the exhaustive search may be an appealing low-complexity 800 approach of finding the optimal solution for our joint opti- 801 mization problem. However, the number of possible cluster 802 formations found by employing the exhaustive search may 803 become excessive with the number of UEs increased. Even 804 if there are only 16 UEs supported by $16 \mathrm{APs}$, the average 805 number of possible formations becomes as high as $5 \times 10^{6} 806$ in a single simulation run. By contrast, the complexity of the 807 KM-algorithm based approach may become inadequate in low- 808 UE-density scenarios. However, when the number of UEs is 809 higher than that of the APs, the complexity is only linearly 810 increased with the number of UEs, according to [26]. Fig. 6b 811 shows both the normalized throughput and the sum utility of 812 various cluster/cell formations, where the traditional NC cell 813 formation designs relying on UFR and on the FR factor of 814 two (FR-2) are considered as our benchmarkers. We adopt the 815 MUS algorithm for the UFR and FR-2 discussed in our previous 816 work [15]. Both the highest throughput attained and the sum 817 utility are quantified for the proposed UC-VT cluster forma- 818 tion, whose optimal solution is found by the exhaustive search. 819 The optimal MWM provides a similar solution as our proposed 820 greedy algorithm, both of which are about $90 \%$ of the opti- 821 mal exhaustive search-based value in the scenario considered. 822 Therefore, we will omit the optimal exhaustive search in the 823 

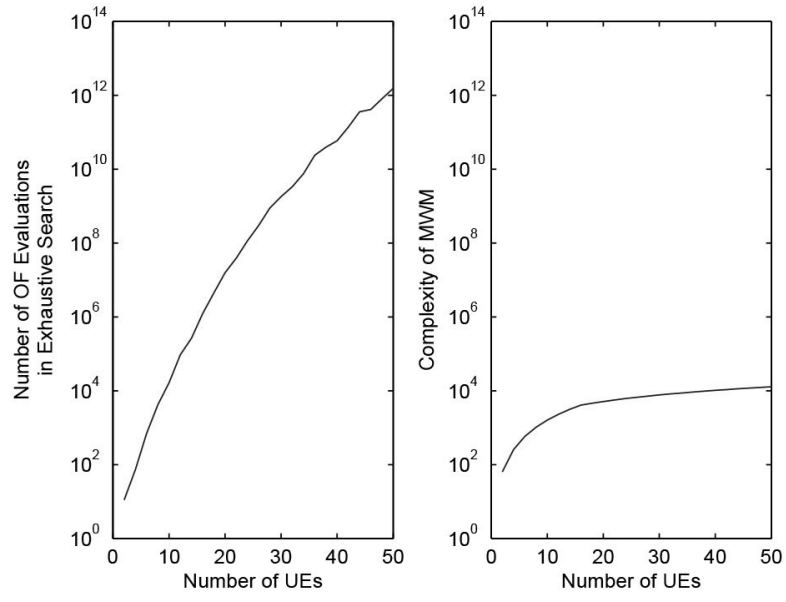

(a)

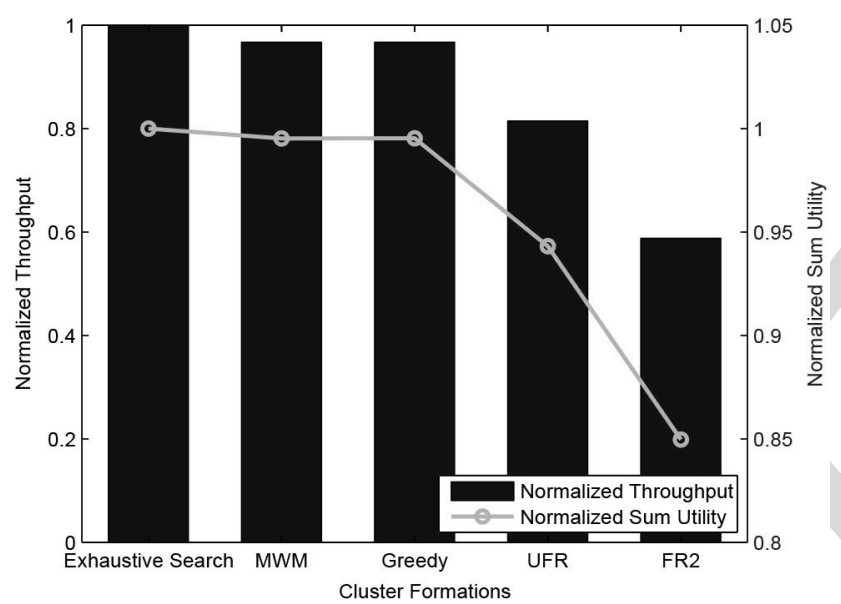

(b)

Fig. 6. (a) The complexity of the exhaustive search for finding the optimal UCVT cluster formations and the complexity of KM-algorithm based MWM for finding a suboptimal cluster formation solution; (b) The normalized throughput and the normalized sum utility/OF value, where $\mathrm{FOV}=110^{\circ}$ and $10 \mathrm{UEs}$ are assumed moving randomly at a speed of $1 \mathrm{~m} / \mathrm{s}$.

rest of this treatise and we opt for the MWM solution as well as for the more practical greedy algorithm for finding the UC-VT cluster formation solution.

\section{B. Throughput Investigations}

1) Throughput Investigations for Various FOV and UE Density: Since the FOV is an influential parameter in VLC networks in Fig. 7a, we consider its effect on the system's performance. The average throughput per UE is reduced, when the $\mathrm{FOV}^{3}$ is increased, due to the increased interference, while our proposed UC-VT cluster formation remains superior in all scenarios considered. In particular, observe in Fig. 7a that the UFR

\footnotetext{
${ }^{3}$ In order to evaluate the system's performance for various FOVs, we selected $110^{\circ} / 115^{\circ}$ and $120^{\circ} / 125^{\circ}$. In the former scenario, the UE is capable of receiving data from two neighboring APs and the area contaminated by potential interference is modest. When the FOV is increased to $120^{\circ} / 125^{\circ}$, the UE is capable of receiving data from four APs and the potential interferencecontaminated area is also increased. These four FOVs correspond to different interference levels, although their absolute values are quite similar.
}

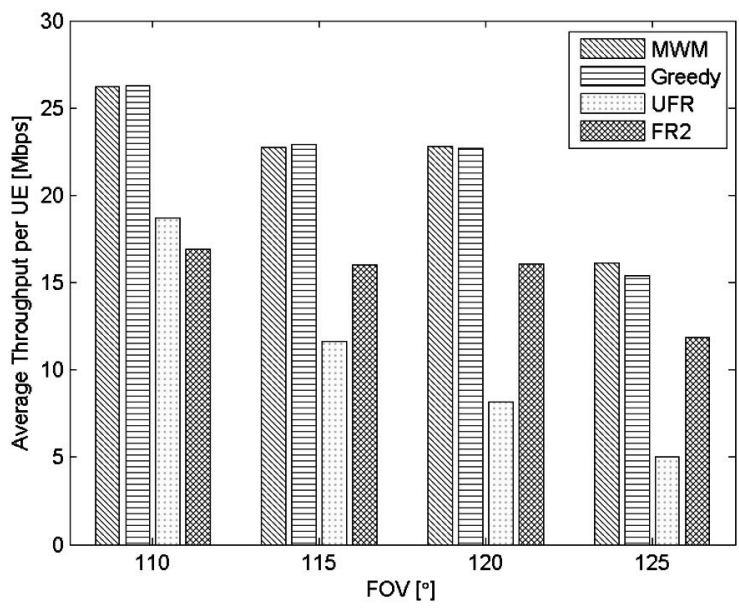

(a)

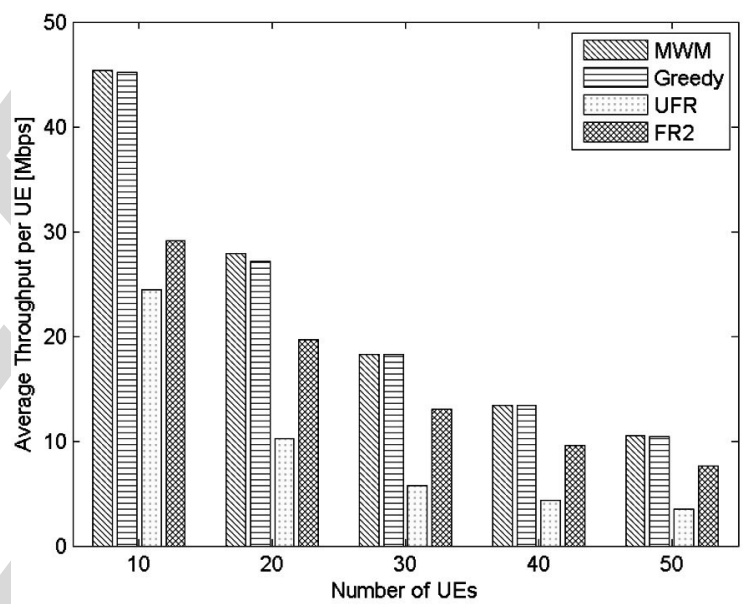

(b)

Fig. 7. (a) Average throughput per UE provided by different cluster formation/cell formation schemes for various FOVs and for 25 UEs. (b) Average throughput per UE provided by different cluster formation/cell formation schemes for various UE densities, where the FOV is $120^{\circ}$ and the number of UEs is 25 .

design exhibits the worst interference immunity and offers the 835 lowest throughput, when the FOV is higher than $115^{\circ}$. Fig. $7 \mathrm{~b} 836$ shows the average throughput per UE provided by different 837 cluster formation/cell formation schemes associated with var- 838 ious UE densities, where the FOV is $120^{\circ}$. As expected, our 839 proposed UC-VT cluster formation is capable of providing the 840 highest average throughput for all the UE densities considered. 841

2) Throughput Investigations for Various LOS Blocking 842 Probabilities: As mentioned in Section I, the performance of 843 VLC systems is expected to be seriously degraded in non-LOS 844 scenarios. In order to investigate the non-LOS behaviour of this 845 VLC system, we introduce the LOS blocking probability $P_{b} 846$ and assume that the achievable data rate $\tilde{R}$ obeys a Bernoulli 847 distribution [13], with the probability mass function of:

$$
f(\tilde{R})=\left\{\begin{array}{lll}
1-P_{b}, & \text { if } & \tilde{R}=R_{s}, \\
P_{b}, & \text { if } & \tilde{R}=R_{r}
\end{array}\right.
$$

where $R_{s}$ and $R_{r}$ denote the achievable data rate of the UE 849 either in the presence or absence of LOS reception. Then the 850 


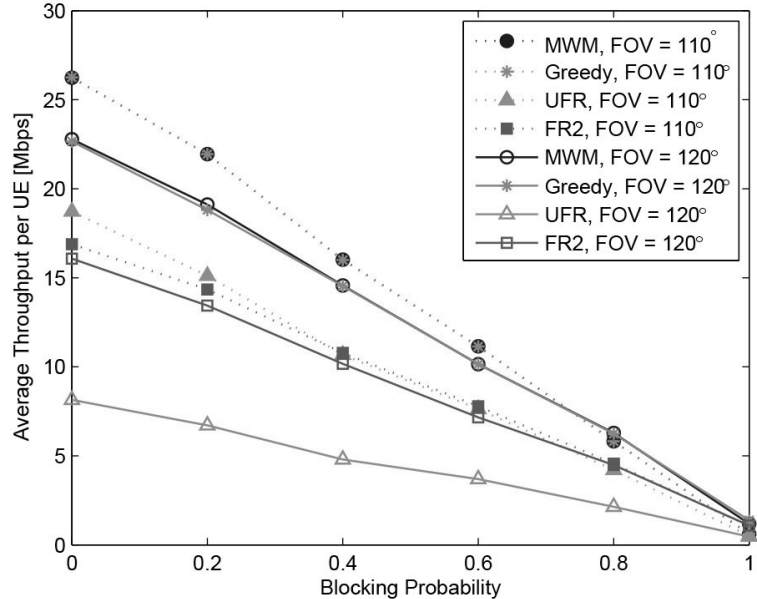

Fig. 8. Average UE throughput of our VLC system for various blocking probabilities and FOVs supporting 25 UEs in each scenario.

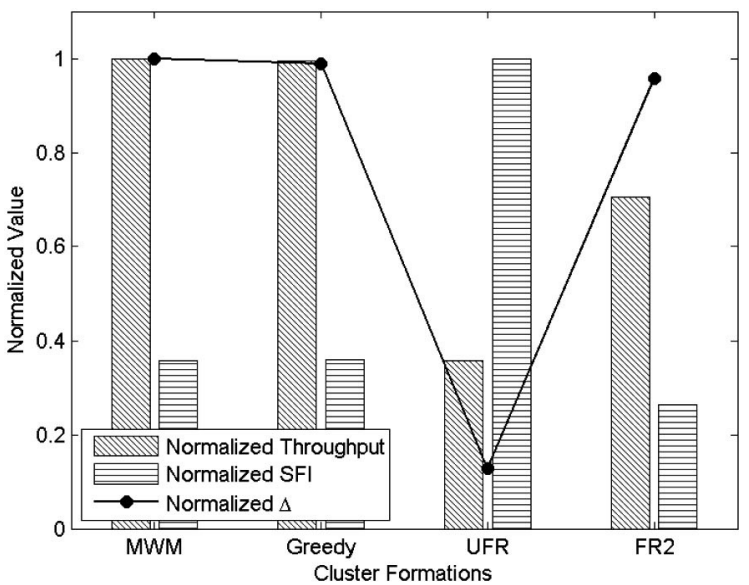

(a)

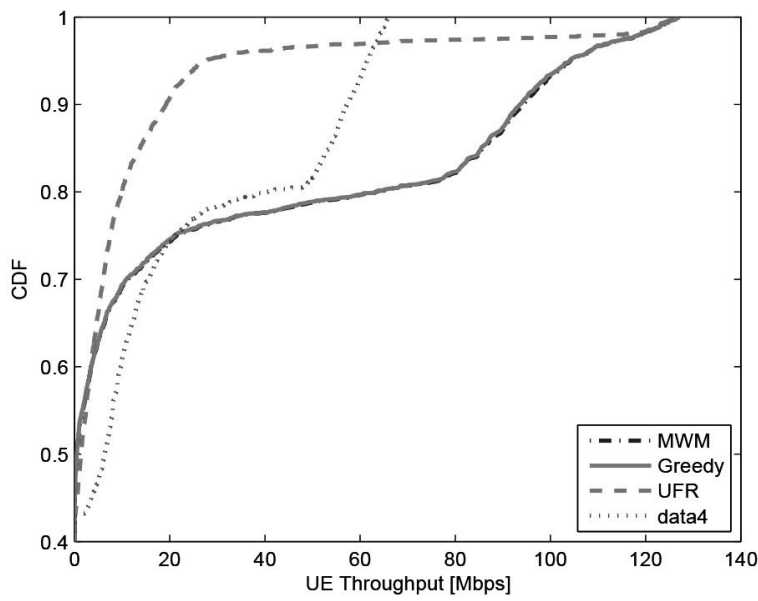

(b)

Fig. 9. (a) The normalized average throughput and the Service Fairness Index (SFI) of various cluster formation/cell formation schemes; (b) CDF of the UE throughput, where the number of UEs is 25 and we have FOV $=120^{\circ}$.

VLC down-link data rate may be written as $\tilde{R}=P_{b} \cdot R_{r}+$ $\left(1-P_{b}\right) \cdot R_{s}$. At this stage, we assume that all LOS paths are blocked with an equal probability. As shown in Fig. 8, the

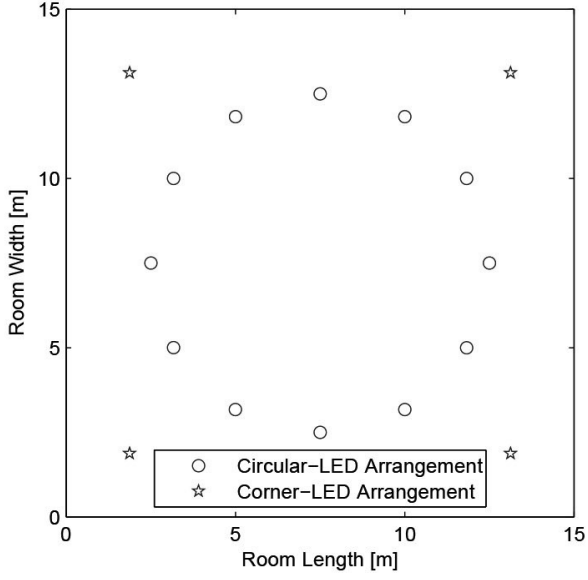

(a)

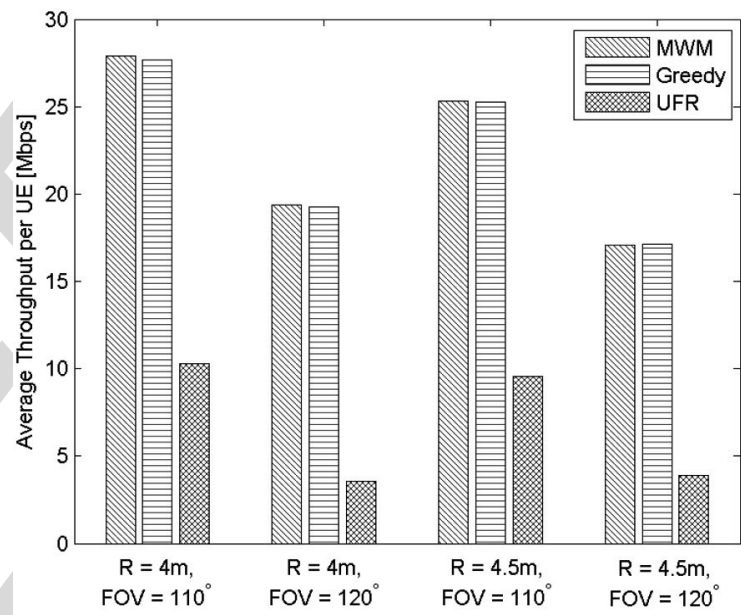

(b)

Fig. 10. (a) shows the LED-arrangement, where the LED circle has a radius of $4.5 \mathrm{~m}$ and the corner LEDs are at $1.875 \mathrm{~m}$ from the walls. (b) System performance of the LED arrangement seen in (a) for 25 UEs.

average UE throughput attained is reduced upon increasing the 854 LOS blocking probability in all the scenarios considered, but 855 our UC-VT cluster formation still achieves a higher throughput. 856 Furthermore, the system performance of the MWM approach 857 and of our proposed greedy cluster formation/MUS algorithm 858 remains quite similar, regardless of the specific blocking prob- 859 ability and FOV.

\section{Fairness Investigations}

In order to investigate the grade of fairness experienced by 862 the UEs, the Service Fairness Index (SFI) of [31] is introduced. 863 The objective of ensuring fairness amongst the UEs is to guar- 864 antee that all UEs benefit from the same throughput within a 865 given period, provided that the UEs' data rate requirements are 866 identical [12], which is often unrealistic. The SFI was defined 867 as [31]: 


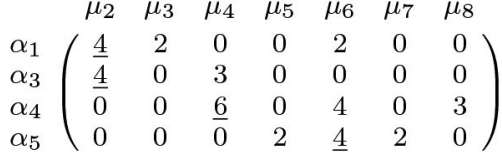

(a)

$$
\left(\begin{array}{ccccccc}
0^{*} & 2 & 4 & 4 & 2 & 4 & 4 \\
0 & 4 & 1 & 4 & 4 & 4 & 4 \\
6 & 6 & 0^{*} & 6 & 2 & 6 & 3 \\
4 & 4 & 4 & 2 & 0^{*} & 2 & 4
\end{array}\right)
$$

(b)

$\left(\begin{array}{ccccccc}0^{*} & 0^{\prime} & 4 & 2 & 2 & 2 & 2 \\ 0 & 2 & 1 & 2 & 4 & 2 & 2 \\ 6 & 4 & 0^{*} & 4 & 2 & 4 & 1 \\ 4 & 2 & 4 & 0 & 0^{*} & 0 & 2\end{array}\right)$

(c) $\left(\begin{array}{ccccccc}0^{*} & 0^{\prime} & 4 & 2 & 2 & 2 & 2 \\ 0^{\prime} & 2 & 1 & 2 & 4 & 2 & 2 \\ 6 & 4 & 0^{*} & 4 & 2 & 4 & 1 \\ 4 & 2 & 4 & 0 & 0^{*} & 0 & 2\end{array}\right) \quad\left(\begin{array}{ccccccc}0 & 0^{*} & 4 & 2 & 2 & 2 & 2 \\ 0^{*} & 2 & 1 & 2 & 4 & 2 & 2 \\ 6 & 4 & 0^{*} & 4 & 2 & 4 & 1 \\ 4 & 2 & 4 & 0 & 0^{*} & 0 & 2\end{array}\right)$

(d)

(e)

Fig. 11. (a) The weight matrix $\left(\omega_{d}\left(e_{\alpha_{i}, \mu_{j}}\right)\right)$ of $\Omega_{2}$, where the maximum element of each row is underlined. (b) Initialization step. The equivalent matrix $\left(a_{\alpha_{i}, \mu_{j}}\right)$ of $\left(\omega_{d}\left(e_{\alpha_{i}, \mu_{j}}\right)\right)$ is obtained as $\left(a_{\alpha_{i}, \mu_{j}}\right)=\left(c_{\alpha_{i}}-\omega_{d}\left(e_{\alpha_{i}, \mu_{j}}\right)\right)$, where we have $\left(c_{\alpha_{i}}\right)=[4,4,6,4]^{\mathrm{T}}$. Find and mark the zero by a star, if there are no starred zeros in its row or in its column. Cover every column containing a $0^{*}$ by a vertical line. (c) Adjustment step. $\left(a_{\alpha_{i}, \mu_{j}}\right)$ is modified as $\left(a_{\alpha_{i}, \mu_{j}}-c_{\alpha_{i}}+\right.$ $\left.r_{\mu_{j}}\right)$, where $\left(c_{\alpha_{i}}\right)=[0,0,0,0]^{\mathrm{T}}$ and $\left(r_{\mu_{j}}\right)=[0,-2,0,-2,0,-2,-2]$. Mark the uncovered zeros by the upper prime. (d) Explicitly, if there is a starred zero in the primed zero's row, mark this row by a line and remove the vertical line for the column of the starred zero. (e) Starred zero and primed zero alternating. Remove all lines. Recover the columns containing $0^{*}$. Optimal solution found.

which reflects the maximum throughput-difference of different UEs. If the SFI is low, the throughput-difference is low and the UEs are served fairly, while if the SFI is high, the UEs experiencing a lower data rate may complain about their unfair treatment. Furthermore, by jointly considering the throughput, we may define

$$
\Delta=\frac{\text { Average throughput per UE }}{\text { SFI }} .
$$

Hence, $\Delta$ constitutes a comprehensive system performance metric, joint characterising both the throughput as well as the service fairness. If $\Delta$ is low, the system either provides a low throughput or a poor fairness; and vice versa. Fig. 9a shows the normalized throughput and SFI of various cell formations and cluster formations, where the UFR design has the worst performance associated with the lowest $\Delta$. Moreover, the Cumulative Distribution Function (CDF) of the UE throughput is shown in Fig. 9b. It can be seen that the UE may have as high as $40 \%$ probability of remaining unserved during each time slot in all the scenarios considered.

\section{Irregular VLC AP Arrangements}

Our proposed UC-VT cluster formation and MUS scheme may be readily applied to arbitrary topologies. Let us consider Fig. 10a, for example. This specific VLC AP arrangement was advocated in [32] for reducing the Signal-to-Noise-Ratio (SNR) fluctuation and was also employed in [16] for implementing a scheduling algorithm. As shown in Fig. 10a, 12 LED lamps constitute a circle and 4 LED lamps are placed in the corners at a height of $2.5 \mathrm{~m}$, which are referred to here as the circular- 894 LED arrangement and corner-LED arrangement, respectively. 895 The power of each LED array is $14.4 \mathrm{~W}$ and $36.8 \mathrm{~W}$ in the 896 circular- and corner-arrangements of our simulations. Thus the 897 total number of optical APs remains 16 and the sum of their 898 transmission power is at most $320 \mathrm{~W}$, which is the same as 899 that of the regular $(4 \times 4)$ LED array arrangement. Fig. 10b 900 shows the average throughput per UE for the LED arrangement 901 of Fig. 10b. The average throughput is slightly reduced, when 902 the radius of the LED circle is increased from $4 \mathrm{~m}$ to $4.5 \mathrm{~m}, 903$ but our proposed UC-VT cluster formation still outperforms the 904 traditional cell formation design in all scenarios of this circular 905 LED arrangement.

\section{CONCLUSIONS}

In this paper, an amorphous UC-VT cluster formation was 908 proposed for mitigating the ICI and to allow a single cluster 909 to support multiple UEs. The MUS problem combined with 910 our UC-VT cluster formation was investigated and the opti- 911 mal solution was found by an exhaustive search approach. 912 Since the exhaustive search may become complex, the origi- 913 nal joint problem was reformulated as a MWM problem, which 914 was solved by the classic KM-algorithm-based method. In 915 order to further reduce the computational complexity, an effi- 916 cient greedy MUS algorithm was proposed for constructing our 917 UC-VT clusters. Our simulation results demonstrated that the 918 UC-VT cluster formation is capable of providing a higher aver- 919 age UE throughput than the traditional NC cell designs in all 920 the scenarios considered. Despite the promise of the UC-VT 921 cluster formation, naturally, some challenges arise when incor- 922 porating our system-level UC design into VLC environments. 923 The open challenges may be highlighted from various perspec- 924 tives, including the acquisition of accurate location information, 925 the research of robustness to LOS blocking, the technology 926 counterpart to be used for up-link support, etc.

\section{APPENDIX A}

\section{KM-ALGORITHM-BASED APPROACH FOR FINDING} THE OPTIMAL MWM

Let us first rely on Lemma 1, where having independent ele- 931 ments indicates that none of them occupies the same row or 932 column.

Lemma 1: (König Theorem)[26]. If $z$ is the maximum num- 934 ber of independent zero elements in the matrix $\left(a_{\alpha_{i}, \mu_{j}}\right)$, then 935 there are $z$ lines (rows, columns or both) containing all the zeros 936 elements of $\left(a_{\alpha_{i}, \mu_{j}}\right)$.

First, the weight matrix $\left(\omega_{d}\left(e_{\alpha_{i}, \mu_{j}}\right)\right)$ of Fig. 3a is formu- 938 lated, as shown in Fig. 11a, where the weight is set to zero 939 when there is no link between two vertices. Our problem is 940 that of maximizing the sum weight, while the KM algorithm 941 is suitable for a minimization problem. We have to construct 942 an equivalent matrix $\left(a_{\alpha_{i}, \mu_{j}}\right)$ for $\left(\omega_{d}\left(e_{\alpha_{i}, \mu_{j}}\right)\right)$, according to 943 Theorem 1. The maximum element $\left(\omega_{d}\left(e_{\alpha_{i}, \mu_{j}}\right)\right)$ is selected and 944 forms $\left(c_{\alpha_{i}}\right)$, where we have $\left(c_{\alpha_{i}}\right)=[4,4,6,4]^{\mathrm{T}}$ in our exam- 945 ple. Let $\left(c_{\alpha_{i}}-\omega_{d}\left(e_{\alpha_{i}, \mu_{j}}\right)\right)$ be the matrix $\left(a_{\alpha_{i}, \mu_{j}}\right)$, as shown in 946 Fig. 11b, and its optimal matching solution minimizing the sum 947 
weight is also optimal for our MWM problem. Next, find a zero in each column of $\left(c_{\alpha_{i}}-\omega_{d}\left(e_{\alpha_{i}, \mu_{j}}\right)\right)$. If however there is no starred zero either in its row or in its column, we mark it by a star, again as shown in Fig. 11b. Then we mark every column containing a $0^{*}$ by a vertical line and all the $0^{*}$ form a set of independent zeros, since none of them occupies the same row or column. The above-mentioned procedure is our initialization step, which may be described as:

i) Initialization. Generate an initial label set $\left(c_{\alpha_{i}}\right)$, where for each row $\alpha_{i}$ we have:

$$
c_{\alpha_{i}}=\max _{\mu_{j}}\left(\omega_{d}\left(e_{\alpha_{i}, \mu_{j}}\right)\right), \quad \mu_{j}=1, \ldots, N_{U, Q_{m}} .
$$

Thus, the equivalent matrix is constructed as $\left(c_{\alpha_{i}}-\right.$ $\left.\omega_{d}\left(e_{\alpha_{i}, \mu_{j}}\right)\right)$. Generate an initial matching $\mathcal{M}_{Q_{m}}$ by finding and marking independent zeros denoted by $z_{j}^{\left(\alpha_{i}, \mu_{j}\right)}$ using a star, whose superscript corresponds to its index in $\left(c_{\alpha_{i}}-\omega_{d}\left(e_{\alpha_{i}, \mu_{j}}\right)\right)$, where we have:

$$
\begin{aligned}
& \forall z_{j}^{\left(\alpha_{i}, \mu_{j}\right)} \in\left(z_{j}\right), \quad z_{j^{\prime} \neq j}^{\left(\alpha_{i}^{\prime} \neq \alpha_{i}, \mu_{j}\right)} \notin\left(z_{j}\right), \\
& \forall z_{j}^{\left(\alpha_{i}, \mu_{j}\right)} \in\left(z_{j}\right), \quad z_{j^{\prime} \neq j}^{\left(\alpha_{i}, p_{\alpha_{i}^{\prime}} \neq \mu_{j}\right)} \notin\left(z_{j}\right) .
\end{aligned}
$$

If $\left|\left(z_{j}\right)\right|=\min \left\{N_{A, Q_{m}}, N_{U, Q_{m}}\right\}$ columns are marked, we find the desired matching, where each AP matches a specific UE and the sum weight of their links is maximized, which furthermore form a UC-VT cluster. Otherwise, the cardinality of the matching will be iteratively increased during the following steps.

If there are no unmarked zeros as shown in Fig. 11b, the current matrix should be modified according to Theorem 1, which leads to the following adjustment step.

ii) Adjustment. Let $h$ be the smallest unmarked element of the matrix and construct a column vector $\left(c_{\alpha_{i}}\right)$ and a row vector $\left(r_{\mu_{j}}\right)$ by the following rules: if the $\alpha_{i}$ th row is covered, $c_{\alpha_{i}}=h$; otherwise, $c_{\alpha_{i}}=0$. If the $\mu_{j}$ th column is covered, $r_{\mu_{j}}=0$; otherwise, $r_{\mu_{j}}=-h$. In our example, $\left(a_{\alpha_{i}, \mu_{j}}\right)$ is updated as $\left(a_{\alpha_{i}, \mu_{j}}-c_{\alpha_{i}}+r_{\mu_{j}}\right)$ and $\left(c_{\alpha_{i}}\right)=$ $[0,0,0,0]^{\mathrm{T}}$ and $\left(r_{p_{i}}\right)=[0,-2,0,-2,0,-2,-2]$, as shown in Fig. 11c.

Then let us choose and mark an unmarked zero by priming it. If there is a starred zero in its row, mark this row by a line and remove the line from the column of the starred zero, as shown in Fig. 11d. Then we prime another unmarked zero in the second row indicated by the bold font, but there is no starred zero in its row. According to the starred and primed zero alternating rules of [26], we obtain the matrix seen in Fig. 11e, where the number of independent zeros reached its maximum given by the number of rows. Correspondingly, the number of lines containing all these zeros becomes maximal, as stated by Lemma 1, where the maximum number of independent zeros is equal to the number of lines containing them. The algorithm terminates here in our scenario. However, if the number of marked columns is still insufficient, the set of independent zeros has to be increased by iteratively repeating the above-mentioned steps, commencing from the Adjustment stage. Thus, we find the optimal solution for our MWM, which is $\left\{\alpha_{10} \rightarrow \mu_{3}, \alpha_{11} \rightarrow \mu_{2}, \alpha_{7} \rightarrow\right.$ $\left.\mu_{4}, \alpha_{6} \rightarrow \mu_{6}\right\}$, namely the same as indicated in Fig. $3 \mathrm{~b}$.
REFERENCES

[1] L. Hanzo, H. Haas, S. Imre, D. O'Brien, M. Rupp, and L. Gyongyosi, 999 "Wireless myths, realities, and futures: From 3G/4G to optical and 1000 quantum wireless," in Proceedings of the IEEE, vol. 100, May 2012, 1001 pp. $1853-1888$.

1002

[2] D. O’Brien, H. Haas, S. Rajbhandari, H. Chun, G. Faulkner, K. Cameron, 1003 A. V. Jalajakumari, R. Henderson, D. Tsonev, M. Ijaz et al., "Integrated 1004 multiple-input multiple-output visible light communications systems: 1005 recent progress and results," in SPIE OPTO, 2015, pp. 93 870P-93 870P. 1006

[3] D. Tsonev, S. Videv, and H. Haas, "Towards a $100 \mathrm{~Gb} / \mathrm{s}$ visible light 1007 wireless access network," Optics Express, vol. 23, no. 2, pp. 1627-1637, 1008 Jan 2015.

1009

[4] D. Tsonev, H. Chun, S. Rajbhandari, J. McKendry, S. Videv, E. Gu, 1010 M. Haji, S. Watson, A. Kelly, G. Faulkner, M. Dawson, H. Haas, and 1011 D. O'Brien, "A 3-Gb/s single-LED OFDM-based wireless VLC link 1012 using a gallium nitride $\mu$ LED," IEEE Photonics Technology Letters, 1013 vol. 26, no. 7, pp. 637-640, April 2014

[5] S. Dissanayake and J. Armstrong, "Comparison of ACO-OFDM, DCO- 1015 OFDM and ADO-OFDM in IM/DD systems," Journal of Lightwave 1016 Technology, vol. 31, no. 7, pp. 1063-1072, Apr. 2013.

1017

[6] A. Azhar, T. Tran, and D. O'Brien, "A Gigabit/s indoor wireless 1018 transmission using MIMO-OFDM visible-light communications," IEEE 1019 Photonics Technology Letters, vol. 25, no. 2, pp. 171-174, Jan 2013.

[7] J. Grubor, S. Randel, K.-D. Langer, and J. Walewski, "Broadband 1021 information broadcasting using LED-based interior lighting," Journal of 1022 Lightwave Technology, vol. 26, no. 24, pp. 3883-3892, Dec. 2008.

[8] J. Armstrong and B. Schmidt, "Comparison of asymmetrically 1024 clipped optical OFDM and DC-biased optical OFDM in AWGN," 1025 Communications Letters, IEEE, vol. 12, no. 5, pp. 343-345, May 1026 2008.

1027

[9] X. Li, R. Mardling, and J. Armstrong, "Channel capacity of IM/DD opti- 1028 cal communication systems and of ACO-OFDM," in IEEE ICC 2007, 1029 June 2007, pp. 2128-2133.

[10] T. Komine and M. Nakagawa, "Fundamental analysis for visible- 1031 light communication system using LED lights," IEEE Transactions on 1032 Consumer Electronics, vol. 50, no. 1, pp. 100-107, Feb. 2004.

[11] "IEEE standard for local and metropolitan area networks-part 15.7: 1034 Short-range wireless optical communication using visible light," IEEE 1035 Std 802.15.7-2011, pp. 1-309, Sep. 2011.

1036

[12] X. Li, R. Zhang, and L. Hanzo, "Cooperative load balancing in 1037 hybrid visible light communications and WiFi," IEEE Transactions on 1038 Communications, vol. PP, no. 99, pp. 1-1, Mar. 2015.

1039

[13] F. Jin, R. Zhang, and L. Hanzo, "Resource allocation under delay- 1040 guarantee constraints for heterogeneous visible-light and RF femtocell," 1041 IEEE Transactions on Wireless Communications, vol. 14, no. 2, pp. 1020- 1042 1034, Feb 2015.

[14] C. Chen, N. Serafimovski, and H. Haas, "Fractional frequency reuse in 1044 optical wireless cellular networks," in IEEE PIMRC 2013, Sep. 2013, 1045 pp. 3594-3598.

1046

[15] X. Li, R. Zhang, J. Wang, and L. Hanzo, "Cell-Centric and User-Centric 1047 Multi-User scheduling in visible light communication aided networks," 1048 in IEEE ICC 2015 (06) ONS, Jun. 2015.

1049

[16] Y. Tao, X. Liang, J. Wang, and C. Zhao, "Scheduling for indoor visi- 1050 ble light communication based on graph theory," Optics Express, vol. 23, 1051 no. 3, pp. 2737-2752, Feb 2015.

[17] R. Zhang, J. Wang, Z. Wang, Z. Xu, C. Zhao, and L. Hanzo, "Visible light 1053 communications in heterogeneous networks: Paving the way for user- 1054 centric design," IEEE Wireless Communications, vol. 22, no. 2, pp. 8-16, 1055 April 2015 .

1056

[18] D. Bykhovsky and S. Arnon, "Multiple access resource allocation in vis- 1057 ible light communication systems," Journal of Lightwave Technology, 1058 vol. 32, no. 8, pp. 1594-1600, April 2014.

[19] M. Biagi, S. Pergoloni, and A. Vegni, "Last: a framework to local- 1060 ize, access, schedule and transmit in indoor VLC systems," Journal of 1061 Lightwave Technology, vol. PP, no. 99, pp. 1-1, 2015.

[20] X. Huang, X. Fu, and W. Xu, "Incremental scheduling scheme for indoor 1063 visible light communication," Electronics Letters, vol. 51, no. 3, pp. 268- 1064 270, Feb 2015.

1065

[21] O. Babatundi, L. Qian, and J. Cheng, "Downlink scheduling in visible 1066 light communications," in WCSP 2014, Oct 2014, pp. 1-6. 1067

[22] H. Kushner and P. Whiting, "Convergence of proportional-fair sharing 1068 algorithms under general conditions," IEEE Transactions on Wireless 1069 Communications, vol. 3, no. 4, pp. 1250-1259, July 2004.

[23] J. Akhtman and L. Hanzo, "Power versus bandwidth-efficiency in wire- 1071 less communications: The economic perspective," in IEEE VTC 2009, 1072 Sep. 2009, pp. 1-5. 


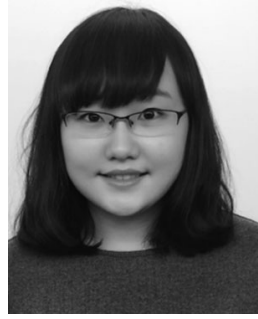

Xuan $\mathbf{L i}$ received the B.Eng. degree in optical information science and technology from Beijing Institute of Technology, Beijing, China, in 2012. She is currently pursuing the Ph.D. degree at the Southampton Wireless Group, University of Southampton, Southampton, U.K. Her research interests include visible light communications, heterogeneous networks, resource allocation, and scheduling algorithms.

1111

1112

1113

1114

1115

1116

1117

1118

1119

1120

1121

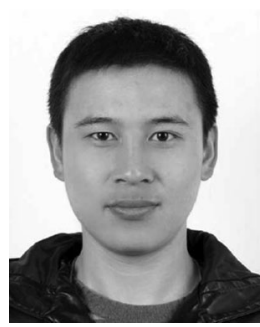

Fan Jin received the B.Sc. degree from Huazhong University of Science and Technology (HUST), Wuhan, China, and the Ph.D. degree from the University of Southampton, Southampton, U.K. in 2010 and 2015, respectively. He is currently working as an Engineer at Huawei, China. He was the recipient of a scholarship under the U.K.-China Scholarships for Excellence Programme. His research interests include multiuser communications, radio resource allocation, spectrum sensing and interference management in femtocells, and heterogeneous networks.

1122

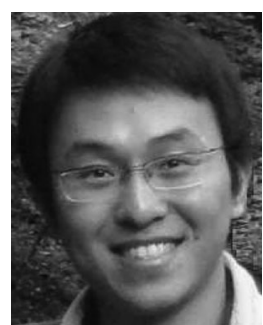

Rong Zhang (M'09) received the B.Sc. degree from the Southeast University, Nanjing, China, and the Ph.D. degree from Southampton University, Southampton, U.K. in 2003 and 2009, respectively. Before obtaining the doctorate, he was an Engineer (August 2003-July 2004) with China Telecom and a Research Assistant (January 2006-May 2009) with Mobile Virtual Center of Excellence (MVCE), U.K. After being a Postdoctoral Researcher (August 2009July 2012) with Southampton University, he took an industrial consulting leave (August 2012-January 2013) for Huawei Sweden R\&D as a System Algorithms Specialist. Since February 2013, he has been a Lecturer with the CSPC Group, ECS, Southampton University. He has authored more than 40 journals in prestigious publication avenues (e.g., IEEE and OSA) and many more in major conference proceedings. He regularly serves as a Reviewer for IEEE transactions/journals and has several times been a TPC member/invited session chair of major conferences. He was the recipient of joint funding from MVCE and EPSRC and is also a Visiting Researcher under the Worldwide University Network (WUN).

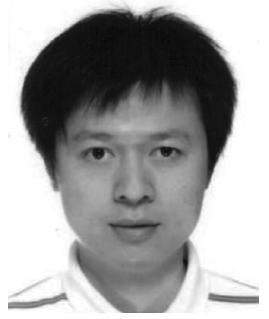

Jiaheng Wang (S'08-M'10-SM'14) received the 1141 B.E. and M.S. degrees from the Southeast University, 1142 Nanjing, China, in 2001 and 2006, respectively, and 1143 the Ph.D. degree in electrical engineering from the 1144 Hong Kong University of Science and Technology, 1145 Kowloon, Hong Kong, in 2010. He is currently 1146 an Associate Professor with the National Mobile 1147 Communications Research Laboratory (NCRL), 1148 Southeast University. From 2010 to 2011, he was 1149 with the Signal Processing Laboratory, ACCESS 1150 Linnaeus Center, KTH Royal Institute of Technology, 1151 Stockholm, Sweden. He also held a Visiting position with the Department 1152 of Computer and Information Science, University of Macau, Macau, China. 1153 His research interests include optimization in signal processing, wireless 1154 communications, and networks. He serves as an Associate Editor for the 1155 IEEE Signal Processing LetTERs. He was the recipient of the Humboldt 1156 Fellowship for Experienced Researchers and the Best Paper Award in WCSP 1157 2014.

1158

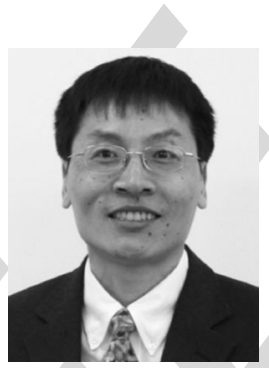

Zhengyuan Xu received the B.S. and M.S. degrees 1159 from Tsinghua University, Beijing, China, in 19891160 and 1991, respectively, and the Ph.D. degree 1161 from Stevens Institute of Technology, Hoboken, 1162 NJ, USA, in 1999. From 1991 to 1996, he was 1163 with Tsinghua Unisplendour Group Corporation, 1164 Tsinghua University, as System Engineer and 1165 Department Manager. In 1999, he joined the 1166 University of California, Riverside, CA, USA, first 1167 as Assistant Professor and then Tenured Associate 1168 Professor and Professor. He was the Founding 1169 Director of the Multicampus Center for Ubiquitous Communication by Light 1170 (UC-Light), University of California. In 2010, he was selected by the 1171 "Thousand Talents Program" of China, appointed as Professor with Tsinghua 1172 University, and then joined the University of Science and Technology of China 1173 (USTC). He is the Founding Director of the Optical Wireless Communication 1174 and Network Center, Founding Director of Wireless-Optical Communications 1175 Key Laboratory of Chinese Academy of Sciences, and Vice Dean of School of 1176 Information Science and Technology, USTC. He is also a Chief Scientist of the 1177 National Key Basic Research Program (973 Program) of China. His research 1178 interests include wireless communication and networking, optical wireless 1179 communications, geolocation, intelligent transportation, and signal processing. 1180 He has authored over 200 journal and conference papers. He has served as an 1181 Associate Editor and Guest Editor for different IEEE and OSA journals. He was 1182 the Founding Chair of IEEE Workshop on Optical Wireless Communications. 1183

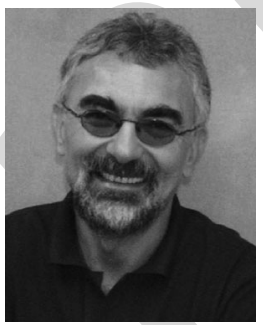

Lajos Hanzo (M'91-SM'92-F'04) received the 1184 D.Sc. degree in electronics and the Doctorate degree 1185 in 1976 and 1983, respectively. In 2009, he was 1186 awarded the honorary doctorate "Doctor Honoris 1187 Causa" by the Technical University of Budapest. 1188 During his 38-year career in telecommunications, 1189 he has held various research and academic posts in 1190 Hungary, Germany, and the U.K. Since 1986, he has 1191 been with the School of Electronics and Computer 1192 Science, University of Southampton, U.K., where he 1193 holds the chair in telecommunications. He has suc- 1194 cessfully supervised about $100 \mathrm{Ph}$.D. students, coauthored 20 Wiley/IEEE 1195 Press books on mobile radio communications totaling in excess of 100001196 pages, published more than 1400 research entries at IEEE Xplore, acted both 1197 as TPC and General Chair of IEEE conferences, presented keynote lectures 1198 and has been awarded a number of distinctions. Currently, he is directing a 1199 100 -strong academic research team, working on a range of research projects 1200 in the field of wireless multimedia communications sponsored by industry, 1201 the Engineering and Physical Sciences Research Council (EPSRC) U.K., the 1202 European Research Council's Advanced Fellow Grant, and the Royal Society's 1203 Wolfson Research Merit Award. He is a Fellow of REng, IET, and EURASIP. 1204 $\mathrm{He}$ is also a Governor of the IEEE VTS. During 2008-2012, he was the Editor- 1205 in-Chief of the IEEE Press and also a Chaired Professor at Tsinghua University, 1206 Beijing. His research is funded by the European Research Council's Senior 1207 Research Fellow Grant. 


\section{QUERIES}

Q1: Please provide post code for the affiliations.

Q2: Please be advised that per instructions from the Communications Society this proof was formatted in Times Roman font and therefore some of the fonts will appear different from the fonts in your originally submitted manuscript. For instance, the math calligraphy font may appear different due to usage of the usepackage[mathcal] \{euscript\}. We are no longer permitted to use Computer Modern fonts. 